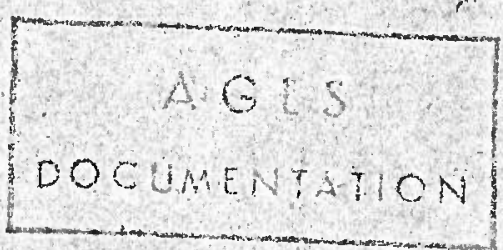

Report to the Government of

AFGMANHSTAN

\title{
SOIL FERTILITY
}

\section{AND FERTILIZER USE}


UNITED NATIONS DEVEIOPMENT PROGRAMME

$1,8.12$

No. TA 3016

$$
\text { il }
$$

R E P O R T

to the

GOVERNMENT

of

AFGHANISTAN

. on

SOIL FERTIIITY AND

FPERTILIZER USE ।

based on the work of

Dr. P.M. Tamboli

Agronomist 
FAO. Report to the Government of Afghanistan on Soil Fertility and Fertilizer Use, based on the work of Dr. P. M. Tamboli, Rome, 1971, 77 p. United Nations Development Programme, [Report] No. TA 3016

\section{ABSTRACT}

Use of chemical fertilizer is a recent development in Afghanistan. The investigations covered finding out the responses to application of chemical fertilizers for wheat, corn, rice and sugarbeet, and estimating the economic dose for optimum yields.

The results indicate, that by the appiication of correct amounts of fertilizers, a farmer can realize a net profit of 300 to 500 Afghanis (US $\$ 1.00$ equals 80 Afghanis), for every 100 Afghanis spent on fertilizers.

The results of the experiments have also been used to project the expected increases in crop production of the country by the modest use of chemical fertilizers, and thus save foreign exchange on import of food.

Tentative fertilizer recommendations for some important crops are included in this report. 
TABLE OF CONTENTS

1. INTRODUCTION

Page.

2. BACKGROUND INFORMATION

2.1 Area, Production and Average Yields of Important Crops

2.2 Soils and Soil Fertility Level

2.3 Fertilizer Imports and Present Consumption, and Estimated Requirements

2.4 Problems of Fertilizer Use

2.5 Climatic Conditions

3. REVIEW OF PAST WORK

3.1 Review of Fertilizèr Trials on Wheat

3.2 Review of Results of Fertilizer Trials on Corn

3.3 Review of Fertilizer Trials on Sugarbeet

4. ACCOMPLISHMENTS

4.1 Fertilizer Trials

4.1.1 Experimental details

4.2 Discussion of Results of Wheat Trials 16

4.2.1 Irrigated wheat

4.3 Fertilizer Trials for Corn

4.4 Experiments on Rice in the Lagman Province

4.5 Sugarbeet Fertilizer Trials

5. ECONOMICS OF FERTILIZER USE IN AFGHANISTAN

6. RECOMMENDATIONS

6.1 Future Outline of Work

6.1.1 General

6.2 Field Programmes to Promote Fertilizer Use

6.3 Applied Research Programmes on Specific Soil Fertility and

6.4 Fertilizer Distribution to Farmers

6.5 Infrastructure for Long-Term Fertilizer Use Development

6.6 Training in Soil Fertility and Fertilizer Work

6.7 Pertilizer Use 
APPRNDICES

Page

RPPENDIX I - REFERENCES

APPENDIX II - ANALYSIS OF VARIANCE

APPENDIX III - SOIL ANALYSIS DATA

KAPPENDIX IV - FRBQUENCY DISTRIBUIION OF SOIL TEST VRIUUS GROUPED

\section{TABLES}

No.

1. Crop Production, Area and Yielas in 1956/67

2. Afghanistan Fertilizer Imports - Ketrto Tons 1961 to 1970

3. Pertilizer Conswiption Per Hectare of irable Land (1966/1967)

4. Fertilizer Trials on Important crops

5. Yield of wheat in Variou Provinces as Insluonced by pertilizer Treatments

6. Average fean Iield of Whot as Affected by Fertilizer Treatments

7. Economics of Fentilizer Application - Whest (1967-1963)

8. Economics of Fertilizer Application - Wheat (1968-1969)

9. Economics of Fertilizer Application - Wheat (1969-1970)

10. Economics of Fertilizer Application - Corn (1968-1969)

11. Economics of Fertilizer Application - Corn (1969-1970)

12. Economics of Fertilizer Application - Rice (1967-1968)

13. Economics of Fertilizer Application - Sugarbeet (1967-1968) 36

14. Tentative Fertilizer Recomendations for the Main Crop for each 36 Province

\section{FIGURES}

Fig. 1 Soils of Afghanistan - Generalized Map 
1. INTRODUCTION

In accordance with a request from the Royal Covernment of Afghanistan for assistance in fertilizer use, the Food and Agriculture Organization of the United Nations, under the United Nations Development Programe, (Technical Asbistance Sector), appointed Dr. P.H. Tamboli as Technical Officer (Soil Fertility). He served in Afghanistan from 24 September 1967 to 31 July 1970. His terms of reference were:

- To study the fertilizer requirements of the main crops on important soil types under irrigated and rain-fed conditions.

- To assemble, analyse and report the results of goil fertility experiments, to interpret these results and develop fortilizer use recomendations.

- To correlate experimental yield data with soil tests and soil survey data, as an aid to develop practical tecomendations.

FAO is reatly indebted to the many people who collaborated with the expert during his Essignment, and who offersd their belpful advice and assistance, especially to:

H.E. Eng. Mir Akbar Reza, and H.E. Abdul Hakim, Ministers of Agriculture and Irrigation; H.E. Dr. Ehsan Rafique, Deputy Minister of Agriculture; Mr. Jumma M. Mohamadi, Presiden of Water and Soil Survey Authority; Mr. A. Ghafoor, President of Research and Ertension Department; Dr. Mohammad Baqaie, Fresident of Irrigation; Mor. Dost Mohamad Nouri, General Director of Solls; Mr. Aminullah and Mr. Asadullah Rudwal, Director of Soil Fertility, and Dr. E.J. Rice of USAID.

\section{BACKGROUND INFORMATTON}

\subsection{Area, Produotion and Average Yields of Important Crops}

In the third five-year economic and social plan of Afghanistan, it is stated that: "Agriculture is the mainstay of Afghan economy, forming the besis of most of the economic activity of the country. More than 85 percent of the population obtain their living from agriculture". Yet, food grains have had to be imported into the country for past decades.

The main problems are: ( $i$ ) that only part of the cultivable area is being used for crop production (out of 14 million hectares of cultivable land, only 7.2 million heotares are under crops), and (ii) that the average yield per hectare is low as compared to other parts of the world.

The annual average crop production, area and yield per heotare are given in Table 1. 
TIJISI

CIDP PRODUCTION ARES AND YIFLDS IN 1966/6I I/

\begin{tabular}{|c|c|c|c|}
\hline Crop & Production & Aroa & Yield \\
\hline & $(1000 \mathrm{mP})$ & $\left(\begin{array}{lll}1 & 000 & h a\end{array}\right)$ & $(\mathrm{kg} / \mathrm{ha})$ \\
\hline Wheat & 2158 & 2343 & 920 \\
\hline Corn & 714 & 500 & 1430 \\
\hline Dariey & 378 & 350 & 1050 \\
\hline Rice (Yilled) & 328 & 214 & 1533 \\
\hline Cotton & 80 & 72 & 167 \\
\hline Sugarbets & 52 & 3.7 & 14865 \\
\hline Sugar cane & 47 & 1.9 & 25263 \\
\hline $0 i 1$ secris & $5 \hat{\imath}$ & 150 & 340 \\
\hline muits & 349 & 47 & 520 \\
\hline Vegetabies & 546 & 104 & 5308 \\
\hline
\end{tabular}

There are inang reasons for the lon yieids, but the important ores are: ( $i$ ) insuficient or Iittle effective fiela preparation and mantenance; (ii) poor imigetion methods or insufficient water; (iii) unsuitable seeding methods; (iv) lack of weed controli ( $v$ ) ise of local low yielding varieties, and, (vi) hardly any return of plent food to the soil, neither sufficient manure, nor (and above all), chemical fertilizers.

\subsection{Soils and Soil Fertility Level}

The central and northeastern mountain cores consist of Pre-ordovician phyllite, Paleozoic sehist, slate, gneiss, granite, grenodiorite and diorite, and Cretaceous, Jurrasic and Coenozoic limestones, sandy marls and shales. Terrestrial (Kuree and Siwalic) formations are also represented in geologic colum. Outcrops of volcanic materials and igneous extrusions are scattered through much of the mountaneous area.

The lowlands of the north, south and east are occupied primarily by Quarternary deposits including alluvial fans, playas and sand dunes, with local loess coverings.

Cultivable soils occur scattered throughout some 40 persent of the total area, mostly at elevations of 1500 to 2700 metres. Sixty percent of the country is in high mountain land, and 40 percent in arid wasteland.

Mountain soils of Sierozom (Calciorthid) chestnut (Ustalf, Ustoll) and Podzolic (Udalf), Brown Forest (Eutrochrept), Alpine Forest (Cryochrept) and Meadow (Cryumbrept) zonea have formed from a complex of residual, colluvial and eolin material.

Sierozem (Caloiorthid, Haplargid) and assooiated soils on rolling hill land of lower altitudes near the cities of Baghlan, Kunduz, Mazar, Maimana, Herat and Panjehir are formed from loess derived from glacial and alluvial materials.

\section{1) Source: Ministry of Agriculture and Irrigation.}


Glacio-fluvial, alluvial and eolian (loesial) sediments form the initial materials of soil of (Camborthids, Haplargids, Ustifluvents, Xerofluvents) the major river valleys.

Desert (Camborthids, Haplargids) soils have developed from both alluvium and sedimentary rocks. The major soil zones are shown in Fig. 1.

The soils are mostly stmactureless, alkaline in reaction and the soil fertility level, in general, is very low. The soils are high in calcium, low in organic matter, low in available nitrogen and phosphorus, and medium to high in available potassium.

Wutrient depleting crops such as wheat, corn, rice, and barley, have been grown for many years without replenishing the nutrient removed. Lack of organic matter has resulted in unfavourable physical condition of the soil for high crop production.

\subsection{Fertilizer Imports and Present Consumption, and Estimated Requirements}

The position with respect to jmports of chemical fertilizer in the country is indicated in Table 2, below.

\section{TABLE 2}

AFGHANISTAN FERTIIIZTR DMPORTS - METRIC TONS 1961 to 1970

\begin{tabular}{|l|c|c|c|c|c|c|c|c|c|}
\hline Fertilizer & $1961-62$ & $1962-63$ & $1963-64$ & $1964-65$ & $1965-66$ & $1966-67$ & $1967-68$ & $1968-69$ & $1969-70$ \\
\hline Ammon. & & & & & \\
$\quad$ Nitrate & 5000 & 7500 & 7500 & - & 2000 & 1700 & - & - & - \\
Urea & - & - & - & - & - & 4200 & 19000 & 6000 & 10000 \\
Super & - & & & & & & & & \\
$\quad$ phosphate & - & 7500 & 7500 & - & - & 2600 & 4000 & 9900 & - \\
DAP & - & - & - & - & - & - & 6650 & 183 & - \\
\hline
\end{tabular}

The two principal sources of fertilizer imports for Afghanistan are the U.S.S.R. and the U.S.A.

It is estimated that urea received from the U.S.S.R. costs US $\$ 99.00$, and super phosphate US $\$ 40.00$ per metric ton, while the urea and DAP from the U.S.A. costs US $\$ 138.00$ and US $\$ 144.00$ per metric ton.

While the urea and di-ammonium phosphate from the U.S.A. costs US $\$ 138$ and 144.40 per metric ton respectively at the rail terminal in Chaman. In addition, the cost of transportation is US $\$ 15$ per ton. $1966 / 67$.

Table 3 gives fertilizer consumption per hectare of arable land in a few countries for 


\section{SOILS OF AFGHANISTAN}

Generalized map, provisional (after Subramanion et al.)

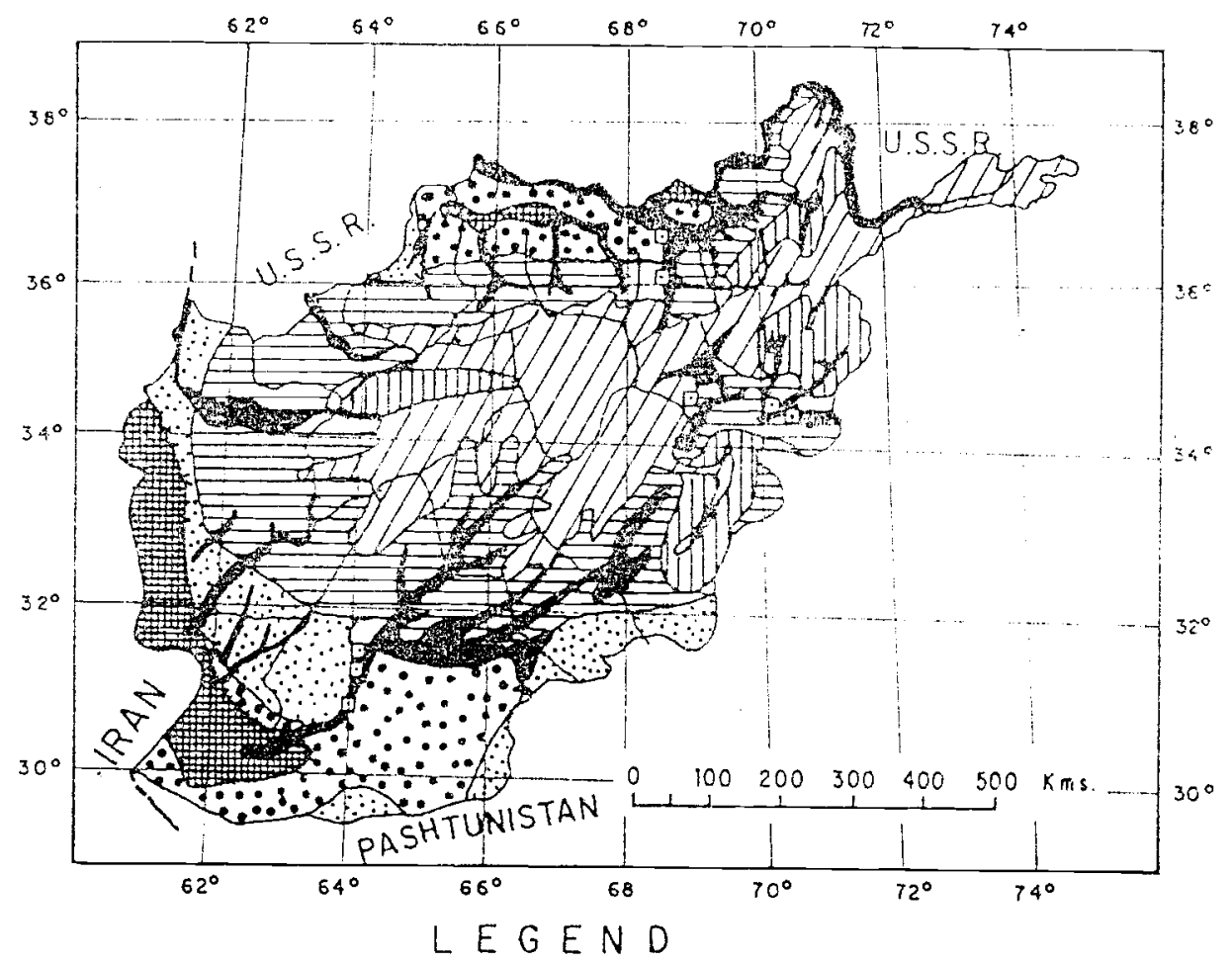

๑: Soil profile site

20. Soils of alluvial plains (Comborthids, Hoplargids \& Ustifluvents)

Soline, Alkoli, \& solt marsh soils (Solorthids, Notrargids \& Haplopuepts)

$\because \because \because \because$ Deserl soils, mostly dunes (Psomments etc.)

Desert soils, with few dunes (Camborthids, etc.)

Sierozem, Brow (Colciorthids \& Hoplorgids) \& Mt. soils with Lithosols \& Regosols (Orthents)

WIIIIII Mountain soils of Chestnut, Podzolic \& Brown Forest zones, with Lithosols (Hoplustolls, Argiustolls, etc.)

ZLD Mountoin soils Forest a Alpine Meadow zones, Glacial Ice a Rock Outcrops (Cryoquepts \& Lilhic groups) 
TABLE 3

FTRTHIZER CONSUMPTION PER HECTARE

OF ARABLE LAND (1966-1967)

\begin{tabular}{|c|c|c|}
\hline Country & Total $\left(\mathrm{N} \cdot \mathrm{P}_{2} \mathrm{O}_{5} \cdot \mathrm{K}_{2} \mathrm{O}\right)$ & Consumption/ha - in $\mathrm{kg}$. \\
\hline Afghanistan & & Less than 1 \\
\hline Belgium & & 620 \\
\hline Netherlands & & 610 \\
\hline United Kingdom & & 221 \\
\hline U.S.S.R. & & 26 \\
\hline U.S.A. & . & 71 \\
\hline India & & 7.6 \\
\hline Japan & & 353 \\
\hline Pakistan & & $7 \cdot 7$ \\
\hline Philippines & & 20 \\
\hline
\end{tabular}

It is estimated that 36000 metric tons of nitrogen and 31000 metric tons of $\mathrm{P}_{2} \mathrm{O}_{5}$ would be required by 1977, for various crops in the country.

At present, all the nitrogeneous and phosphetic fertilizers are imported into the country. The urea plant at Mazar-i-Sharif is expected to be comnissioned between 1971 and 1972 , with a capacity of 105000 tons of urea per year.

\subsection{Problems of Fertilizer Use}

As already indicated, the use of chemical fertilizers has started only recently in Afghanistan. Generally, the farmers have not taken up a too intensive use of chemical fertilizers, due to the following reasons:

a) hesitance of farmers to invest money, or simple lack of cash;

b) non-availability of fertilizers;

c) shortage of irrigation facilities, and

d) lack of knowledge to use this input economically.

Furthermore, it must be remembered that fertilizers are economically effective only if the correct kind and amounts are used for each local kind of soil.

The required basic data to make specifio fertilizer recommendations are not available, therefore the Bxtension Agents find it difficult to convinoe the farmers.

\subsection{Glimatic Conditions}

Afghanistan is located in the low latitude desert and steppe belt of the Asiatio olimatio system. The olimate is aharacterized by a subtropical high pressure zone of subsiding air and influenced by the cold, dry northeast winds from the interior of Asia in the winter, and by warming, dessicating west and northwest winds on the western side 
of the ABiatic low lands in the summer. Yhost of the precipitation in the country results from orographic uplist of air mases. Brtents of eneralized climatic types are shown in Fig. 2 .

The complex amangenents of mountain slopes, valleys and pleins in the country result: in an intricate micro-climatic pattern. Average annual precipitation is given below.

\begin{tabular}{|c|c|c|c|c|}
\hline & $\begin{array}{l}\text { Average Annual } \\
\text { Precipitation }\end{array}$ & & Average & Annual Temp. ${ }^{\circ} \mathrm{C}$ \\
\hline Location & in Inches & No of Years & $\ln 3 x$ & Min. \\
\hline Bagelian & 9.9 & 13 & 24 & -2 \\
\hline Charni & 12.9 & 18 & 20 & -10 \\
\hline Parran & 15.8 & 6 & 25 & -4 \\
\hline vingrahar & 6.2 & $2 \hat{1}$ & 36 & +5 \\
\hline Kavil? & 13.3 & 10 & 24 & -4 \\
\hline Ingsin & 15.9 & 2 & 36 & +5 \\
\hline
\end{tabular}

\section{DEMEN OS FAST HORE}

Recently, a few agronomic fertilizer experiments have been conducted in the country on some important crops, but these experiments havo been: (i) too few in number;

(ii) often not statistically designed, and (iii) have not been repeated for three seasons. Further, the results of these experiments have not been correlated with soil survey and soil testing data.

A short review of fertilizer experimental work done so far for each crop is given in the following section.

\subsection{Review of Fertilizer Trials on Wheat}

In 1951, Dr. Hauser initiated a soil fertility investigation programme in Afghanistan. on the basis of his studies he concluded that: (a) nitrogen is a limiting factor in production in most Afghan solis; (b) soils, if low in nitrogen, application of phosphorus alone will not help; (c) application of phosphorus on solls rich in nitrogen is beneficial; (d) potassium is not a limiting factor in Afghanistan; (e) soils having more than 5ppm of water soluble phosphorus will not respond to applioation of phosphorus, and ( $f$ ) one or two heavy applications of local manure did not correct deficiency of phosphorus.

After 1951, for a period of about nine yeara, no work has been reported on fertilizer responses. Frutchey (1960), reported that on Damal Aman Farm, Kabul, good response to application of high doses of phosphorus is observed. He also found that use of nitrogen alone decreased the yield. There was wide variability in the data reported by him and the results were not conolusive.

Shuman (1960), worked on wheat in Kabul Province, and found that in Paghman, Chilstoon and Slab areas, application of 15 pounds $\mathrm{N}$ and 15 pounds $\mathrm{P}_{2} \mathrm{O}_{5}$ per jerib $1 /$ $(34-34-\mathrm{kg} / \mathrm{ha})$. resulted in 149 percent incresse in jield over control. 5 He reported thet in Chauky and Bagrami areas, by the epplication of $50 \mathrm{~N}+20 \mathrm{P}_{2} \mathrm{O}_{5} \mathrm{~kg} / \mathrm{ha}$, gave a net profit of 242 Afghanis.

\section{$1 / 1$ jerib equals 0.1936 heotere}




\section{FIGURE 2}

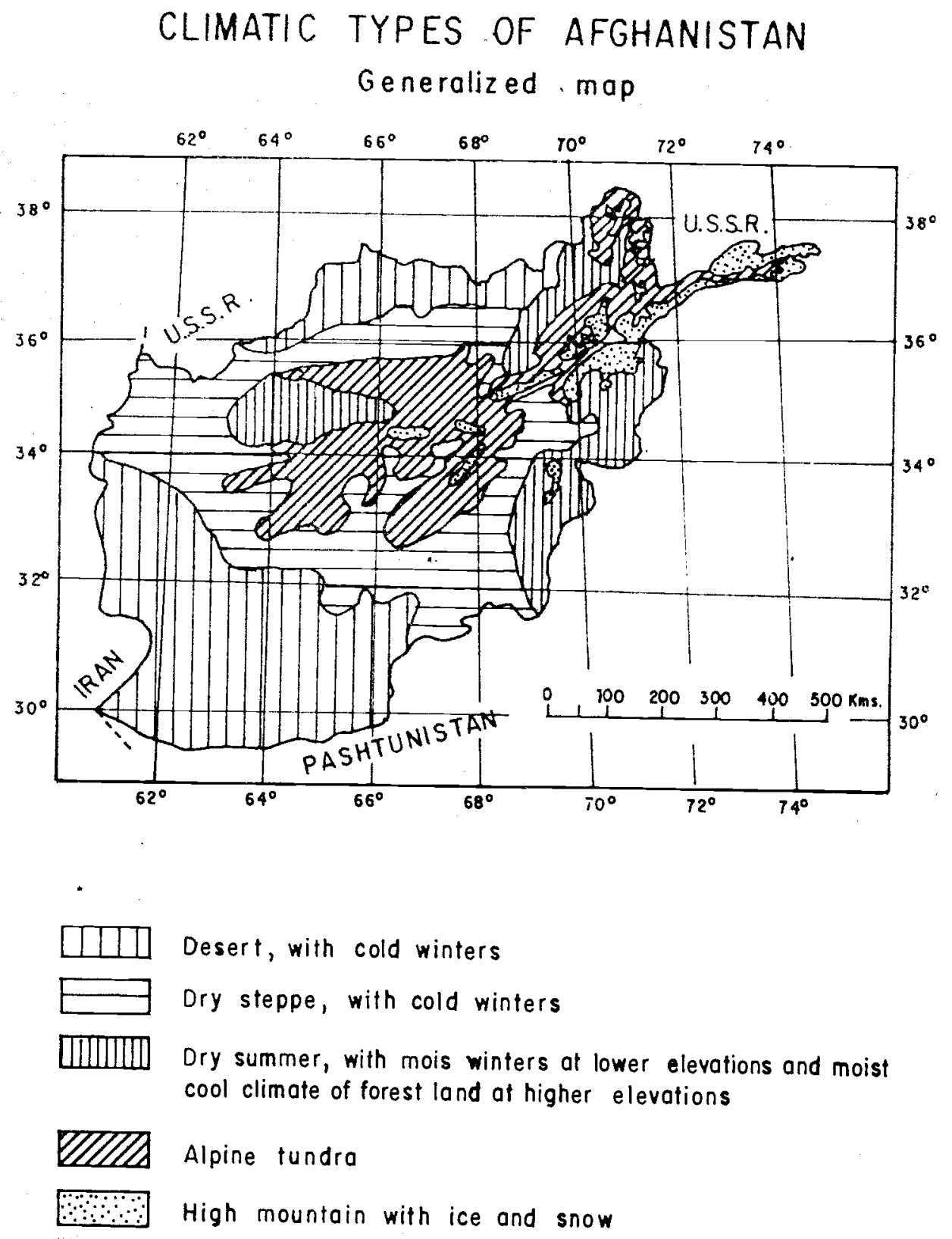

U.G. 
Nelson (1962), found that application of 3 aeers $2 /$ of urea, 6 seers of superphosphate and 2 geers of potassium chloride per jerin $(46-42-42 \mathrm{ke} / \mathrm{ha})$, gave 76 percent increase in wheat yield. In 1965; Kunler conducted pot culture experiments on some soils and found that $40 \mathrm{P}, \mathrm{O}$ pounds per aore $(40-40 \mathrm{~kg} / \mathrm{ha})$, was a most eutable dose. He also found that soils of vary low and medlum fortility responded ecrally to addition of nitrogen and phospherus.

Carter (1966), worked in Heimand Valley Anthority and obtained 150 pounds increase over control (22 percent), by addition of 18 pounds $N$ and 9 pounds $P_{2} O_{5}$ per jerib $(4020 \mathrm{~kg} / \mathrm{ha})$. Samin and Nielsen $(12)$, oonducted about 140 individual frials in the Jalalabed, Baghlan and Kabul area. They found that uree and treble phosphate applied at the rate of 85 kilogrames each of nitrogen and $\mathrm{P}_{2} \mathrm{O}_{5}$ per hectare, increased wheat yiela by 32 and 40 percent at the Hinistry of Agriculture Stations at Jalalahad and Eaghlan, respectively. In the Kabul area, $85 \mathrm{kilogramnes}$ of nitrogen and $165 \mathrm{ki}$ iogrammes of $P_{2} O_{5}$ increased yield by 10 percent. They also reported avidence of P-firstion problem in ${ }^{2}$ the Baghlan area, where soils with only $4.0 \mathrm{ppm}$ available phosphorus did not respond to phosphorus application. Mortensen (1967), reported the higtest yield of $5966 \mathrm{~kg} / \mathrm{ha}$ by application of $90 \mathrm{~N}+45 \mathrm{P}_{2} \mathrm{O}_{5} \mathrm{~kg} / \mathrm{ha}$ at the Jalalabs Research Station.

U:der the Accelerated wheat Progreme (jointly sponsored by the Government and USAID in 1968), uniform fertiliser trials were conducted at the Research Stations of Kabul:

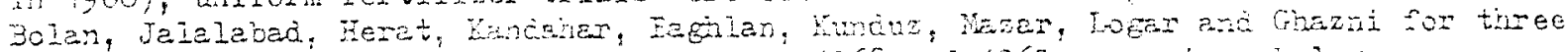
years. The treatment and everage yelds for 1068 and 1969 , are Eiven belon.

\begin{tabular}{|c|c|c|}
\hline & $\because \frac{150 / 4 a}{205}$ & Avarage yiejas/J \\
\hline 1. & $0 \ldots 0$ & 60 \\
\hline 2 . & $0-40$ & 60 \\
\hline 3. & $0-60$ & 69 \\
\hline 4. & $0-80$ & 72 \\
\hline 5. & $0-100$ & 66 \\
\hline 6. & $67-0$ & 79 \\
\hline $7 \cdot$ & $67-40$ & 88 \\
\hline 8. & $67-60$ & 93 \\
\hline 9. & $67-80$ & 90 \\
\hline 10. & $67-100$ & 93 \\
\hline 11. & $133-0$ & 87 \\
\hline 12. & $133-40$ & 102 \\
\hline 13. & $133-60$ & 100 \\
\hline 14 . & $133-80$ & 106 \\
\hline 15. & $133-100$ & 113 (maximum profit) \\
\hline 16. & $200-0$ & 85 \\
\hline 17. & $200-40$ & 104 \\
\hline 18. & $200-60$ & 112 \\
\hline 19. & $200-80$ & 119 \\
\hline 20. & $200-100$ & 120 \\
\hline
\end{tabular}


Since these experiments did not Give consistent resuits for three years, it is proposed to continue them for one more year.

The maximum profit was obtained by treatment No. $15-i . e ., 133-100 \mathrm{~N}-\mathrm{P}_{2} \mathrm{O}_{5} \mathrm{~kg} / \mathrm{ha}$. :

\subsection{Review of Results of Fertilizer Trials on Corn}

The work of fertilizer trials on corm was initiated by Shuman (1969). He conducted non-replicated experiments in the $\mathrm{K}_{\mathrm{Bbul}}$ area and found that chemical fertilizers increased the average yield by about $1331 \mathrm{~kg} / \mathrm{ha}$. The results of his experiments are summarized as follows:

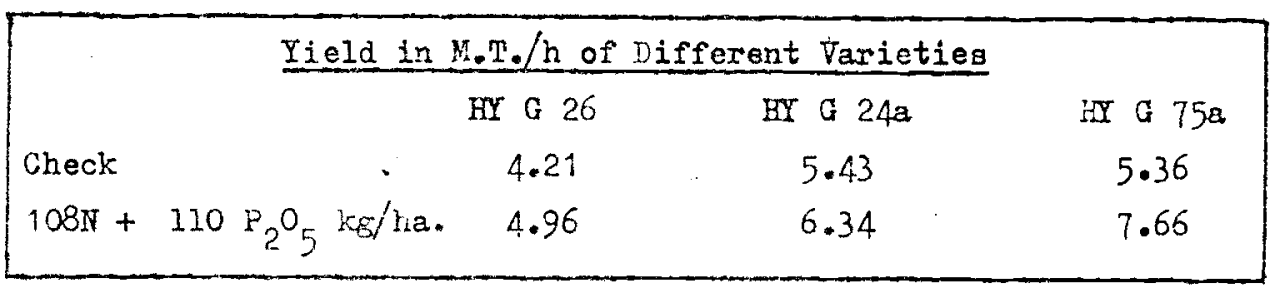

Vaugh (1961) conducted some trials in the Heimand Veiley during 1961 and obtained a yieic of $4.99 \mathrm{M}$.T. $/ \mathrm{h}$ by the use of $100 \mathrm{iN}+67 \mathrm{P}_{2} \mathrm{O}_{5} \mathrm{~kg} / \mathrm{h}$, when the yield of unfertilized plot was onity $2.34 \mathrm{M} . \mathrm{T} . \mathrm{h}$.

Wielsen (1964) tried Mimesota 13 - 13 variety in 1964 and reported that at Aliabad farm, the unfertilized plot gave a yield of $2.98 \mathrm{M.T} . / \mathrm{h}$ of corn as compared to $3.67 \mathrm{M} . \mathrm{T} . / \mathrm{h}$ from the fertilized plots where $207 \mathrm{~N}+207 \mathrm{P}_{2} \mathrm{O}_{5} \mathrm{~kg} / \mathrm{h}$ were added.

The rates used by the authors would prove uneconomical at the present time.

While at Darulaman farm, the yield of check plot was $3.52 \mathrm{M} \cdot \mathrm{T} \cdot / \mathrm{h}$, and the fertilized plot yielded $6.24 \mathrm{M} \cdot \mathrm{T} \cdot / \mathrm{h}$ where $205 \mathrm{~N}+180 \mathrm{P}_{2} \mathrm{O}_{5} \mathrm{~kg} / \mathrm{h}$ was added.

Samin $(1965-66)$ reported a yield of 3.52 and $6.84 \mathrm{M} . \mathrm{T} \cdot / \mathrm{h}$ in the Kabul and Shawaki areas respectively, where 500 donkey loads of manure were added per hectare.

Carter (1967), compared the yields of fertilized and unfertilized plots of local and surcropper variety in the Helmand Valley during 1967. The results reported by him are summarized as follows:

\begin{tabular}{|lcc|}
\hline & Yield in $\mathrm{M.T} . / \mathrm{h}$ of Tho Varieties \\
\cline { 2 - 3 } & Local & Suroropper \\
Check & 1.21 & 2.47 \\
$52 \mathrm{~N}+47 \mathrm{P}_{2} \mathrm{O}_{5} \mathrm{~kg} / \mathrm{h}$ & 1.60 & 3.03 \\
\hline
\end{tabular}

Samin and First (1968), obtained highest yield of 4.7 and $5.95 \mathrm{M.T.} / \mathrm{h}$ on fields 2 and 5 respectively at the Darulaman farm by adding $104 \mathrm{~N}+104 \mathrm{P}_{2} \mathrm{O}_{5} \mathrm{~kg} / \mathrm{h}$. The yields from the contr plots from these two fields being 3.3 and $4.2 \mathrm{M.T} / \mathrm{h}$, respectively.

Nattaqi and Nielsen (1968), compared the looal and Minnesota variety at Darulaman farm. They applied $50.7 \mathrm{~N}+53.5 \mathrm{P}_{2} \mathrm{O}_{5} \mathrm{~kg} / \mathrm{h}$ and obtained highest yield of 3.31 and $3.20 \mathrm{M} . \mathrm{T} . / \mathrm{h}$ of
the local and $\mathrm{M}-13$ variety. 


\subsection{Reviow of Fertilizer Trials on Sugerbeet}

A very valuable pioneering rork was done by Irr. Hauser on sugarbeet cultivation about 16 to 17 years ago. He conducted experiments on plenting mothods and proved that the Juia liethod Gives poor yield as compared to flat soil planting. The advantagos of the latter method being high yield, less work for the farmor, quicker and effective reeding, better $A$ germination etc. He therefore strongly recomended that all the suparibet fields should be levelied in terraces, and planting of beet on sloping ijelds should be prohibited. Fe also conducted replicated fertilizer trials on sugarbet and his resulta are sumarized below:

\begin{tabular}{|c|c|c|c|}
\hline Treatment & Beet Yielde in tons $/ \mathrm{h}$ & Percentafe of Check & $\begin{array}{c}\text { Percentage of Surar } \\
\text { in Reet }\end{array}$ \\
\hline Check & 30.95 & 100 & 18.35 \\
\hline $80-0-0$ & 41.80 & 135 & 17.95 \\
\hline $0-54-0$ & 42.80 & 139 & 18.45 \\
\hline $80-54-0$ & 50.85 & 164 & 18.65 \\
\hline $\begin{array}{l}2450 \mathrm{sg} / \mathrm{h} \\
\text { Cottor care }\end{array}$ & 40.25 & 30 & 18.50 \\
\hline $\begin{array}{l}2450 \mathrm{rgh} \\
3 \mathrm{het}\end{array}$ & 30.25 & ce & 13.10 \\
\hline
\end{tabular}

The results of his experimerts indicated that the averege 3 ield of the experimental fields was 40.70 tons $/ \mathrm{h}(8.14$ tons $/ \mathrm{J})$ whereas the average yield on the farmers field was 7.50 tons $/ \mathrm{h}(1.50$ tons $/ \mathrm{J})$. The average sugar content of the experimentel plots was 18.4 percentas compared to 15.5 percent in the beets from the farmers' fields. It is unfortunate that this work was not followed up. During 1967 an experiment was conducted at the research: farm in Juianfields at Baghlan. The rosults are summarized below:

\begin{tabular}{|lc|}
\hline Treatment & Yields of Beet in tons $/ h$ \\
$0-0-0$ & 4.66 \\
$0-60-0$ & 6.35 \\
$60-90-0$ & 9.50 \\
$60-0-0$ & 7.25 \\
$80-120-0$ & 11.90 \\
$160-150-0$ & 13.20 \\
\hline
\end{tabular}

The soil analysis data for these fields are not available, and the results are for one season only.

This scanty information indicates very clearly that there is no precise knowledge of the effect of fertilizers on sugarbeet. 


\section{ACCOMPLISHMENTS}

\subsection{Fertilizer Trials}

Keeping in view the objectives of the project, sbout 743 fertilizer trials were conducted on important orops in some regions of Afghanistan on farmers' fields. The year, location, crop and number of trials are sumnerized in Table 4 .

TABLE 4

FWRTIITER TRIALS ON IMPORTANT CROPS

\begin{tabular}{|c|c|c|c|}
\hline Tear & Location & Crop & No. of trials \\
\hline $1967-68$ & $\begin{array}{l}\text { Kabul, Logar, Maidan, } \\
\text { Parwen, Ghazni, Paktia }\end{array}$ & Wheat & 25 \\
\hline $1967-68$ & Logar & Corn & 10 \\
\hline $1967-68$ & Baghlan & Sugarbeet & 10 \\
\hline $1967-68$ & Laghman & Ricos & 10 \\
\hline $1968-69$ & $\begin{array}{l}\text { Kabul, Logar, Kaidan, } \\
\text { Kapisa, Parwan, Laghman, } \\
\text { Ningrahar }\end{array}$ & iheat & 192 \\
\hline $1968-69$ & Logar, Paktia & Com & 75 \\
\hline \multirow[t]{2}{*}{$1969-70$} & $\begin{array}{l}\text { Kabul, Logar, Kaidan, } \\
\text { Karisa, Parwan, Baghlan, } \\
\text { Ghazni, Ningrahar, } \\
\text { Laghman and Kunar }\end{array}$ & Wheat & 421 \\
\hline & & \multicolumn{2}{|c|}{ Total 743} \\
\hline
\end{tabular}

Soil samples from exch of the experimentsl plots were collected before the application of chemical fertilizers. They have been analysed for their nutrient oontents (1.e., available $\mathrm{N}, \mathrm{P}_{2} \mathrm{O}_{5}$ and $\mathrm{K}_{2} \mathrm{O}$; $\mathrm{pH}$; conductivity; texture and $\mathrm{CaCO}_{3}$ content). The soil analysis data are presented in Appendix II.

\subsubsection{Experimental details}

In all the experiments, the source of $\mathrm{N}$ was urea ( 46 percent), and that of $\mathrm{P}_{2} \mathrm{O}_{5}$ and $\mathrm{K}_{2} \mathrm{O}$ super-phosphate $\left(18\right.$ peroent $\left.\mathrm{P}_{2} \mathrm{O}_{5}\right)$, and potgssium sulphate ( 60 percent $\left.\mathrm{K}_{2} 0\right)$, respectively. Also the plot size was uniformly th 5 ame $-50^{2} \mathrm{~m}(10 \mathrm{x} \mathrm{m})$, in all the experiments.

a) Results of Wheat Trials - during 1967 and 1968, the following treatments were used: 


\begin{tabular}{|c|c|c|c|}
\hline Thea tment No. & $\begin{array}{c}\text { Dry Land } \\
\text { lrig/ha }\end{array}$ & Thertment No. & $\begin{array}{c}\text { Irrigated } \\
\mathrm{kg} / \mathrm{ha}\end{array}$ \\
\hline & $H-P_{2} O_{5}$ & & $N-P_{2} O_{5}$ \\
\hline 1 & $0-0$ & 1 & $0-0$ \\
\hline 2 & $15-0$ & 2 & $15-0$ \\
\hline 3 & $30-0$ & 3 & $30-0$ \\
\hline 4 & $0-15$ & 4 & $60-0$ \\
\hline 5 & $0-30$ & 5 & $0-15$ \\
\hline 6 & $15-15$ & 6 & $0-30$ \\
\hline 7 & $30-30$ & 7 & $0-60$ \\
\hline & & 8 & $15-15$ \\
\hline & & 9 & $30-30$ \\
\hline & $:$ & 10 & $60-60$ \\
\hline
\end{tabular}

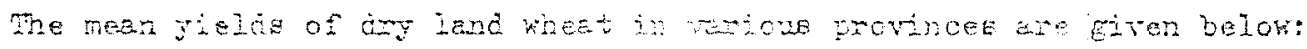

\begin{tabular}{|c|c|c|c|c|c|}
\hline Irestirent No. & Yeru & Eicar & Chang & Detie & Exaran \\
\hline$i$ & 167.5 & 1945 & 333.6 & 237.7 & 257.6 \\
\hline 2 & 381.6 & 313.3 & 459.0 & 402.5 & 484.6 \\
\hline 3 & 566.0 & 352.3 & 663.8 & 560.5 & 552.6 \\
\hline 4 & 240.0 & 394.0 & 337.8 & 355.5 & 556.0 \\
\hline 5 & 343.0 & 476.3 & 402.5 & 354.2 & 566.0 \\
\hline 6 & 610.0 & 718.3 & 747.5 & 740.2 & 793.0 \\
\hline 7 & 815.6 & 1008.0 & 1110.0 & 1035.0 & 872.6 \\
\hline
\end{tabular}

IF Test was signifloant in all the casas.

C.D. $\quad \begin{array}{lllll}56.07 & 55.76 & 88.06 & 129.27 & 62.47\end{array}$

The mean yields of irrigated wheat in Kabul and Logar Provinces are as follows: 


\begin{tabular}{|ccc|}
\hline Treatment & $\frac{\text { Kabul }}{468.6}$ & $\frac{\text { Logar }}{428.3}$ \\
\hline 1 & 568.0 & 455.3 \\
2 & 696.3 & 592.0 \\
3 & 773.3 & 675.6 \\
4 & 398.0 & 442.0 \\
5 & 469.0 & 584.0 \\
6 & 568.0 & 644.0 \\
7 & 1117.3 & 168.6 \\
8 & 1906.6 & 2140.3 \\
9 & 2396.3 & 2390.0 \\
10 & & \\
\hline F' Test Significant & 140.9 & 122.4 \\
CD & & \\
\hline
\end{tabular}

In the year 1968-69 and 1969-70 the following treatments were used, and results are given in Table 5 .

\begin{tabular}{|crcr|}
\hline Treatment No. & $\underline{N}$ & $\frac{\mathrm{kg} / \mathrm{ha}}{\mathrm{P}_{2} \mathrm{O}_{5}}$ & $\underline{\mathrm{K}}_{2} \mathrm{O}$ \\
1 & 0 & 0 & 0 \\
2 & 75 & 0 & 0 \\
3 & 150 & 0 & 0 \\
4 & 0 & 75 & 0 \\
5 & 0 & 150 & 0 \\
6 & 75 & 75 & 0 \\
7 & 75 & 150 & 0 \\
8 & 150 & 75 & 0 \\
9 & 150 & 150 & 0 \\
10 & 0 & 75 & 100 \\
11 & 75 & 75 & 100 \\
12 & 150 & 150 & 100 \\
\hline
\end{tabular}


TABLE 5

YITD OF WHFAT IN VARIOUS PROTINCBS AS INMUUENED BX FERTILIZER TREATHEATS (kg/ha)

\begin{tabular}{|c|c|c|c|c|c|c|c|}
\hline \multicolumn{2}{|r|}{ Treatment } & Lafman & Nangarhar & Kebul & $\begin{array}{c}\text { Kapisa and } \\
\text { Parwan }\end{array}$ & Logar & Maidan \\
\hline & $\mathrm{H}-\mathrm{P}_{2} \mathrm{O}_{5}-\mathrm{K}_{2} \mathrm{O}$ & & & & & & \\
\hline 1. & $0-$ & 1167 & 1087 & 1655 & 1296 & 1378 & 1089 \\
\hline 2. & $0-0$ & 1542 & 1406 & 1814 & $i 574$ & 1555 & 1290 \\
\hline 3. & $0-0$ & 1800 & $1 T 10$ & 2031 & 1738 & 1727 & 1441 \\
\hline 4. & $0-75-0$ & 1.427 & 1310 & 1985 & 570 & 1637 & 1367 \\
\hline 5. & $0-150-0$ & 1540 & 1390 & 2148 & 1580 & $172 \hat{\imath}$ & $: 4 E 0$ \\
\hline 6. & $75-75-0$ & 2281 & 2865 & 3132 & $2 \div 9$ & 2539 & 2776 \\
\hline 7. & $75-150-0$ & 2052 & 1554 & $=550$ & 23.5 & 2024 & 248 \\
\hline 8. & $150-75-0$ & 2292 & 2.30 & 2596 & 2322 & 2168 & 2428 \\
\hline 9. & $150-150-0$ & $\equiv 197$ & 3304 & 5420 & $4 \div 60$ & 4760 & $\therefore 900$ \\
\hline 10. & $0-75-100$ & 1704 & 1321 & 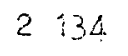 & 20,9 & 1684 & 1407 \\
\hline 11. & $75-75-100$ & 2354 & 2319 & 3158 & 2711 & 2591 & 2487 \\
\hline 12. & $150-150-100$ & 3310 & 3376 & 5573 & 3968 & 4618 & 4690 \\
\hline
\end{tabular}
b) Results of Dry Land wheat experiments in Kabul $1968-691 /$ the results of these

\begin{tabular}{|cc|}
\hline Treatment No. & $\frac{\text { Yield kg/ha }}{440}$ \\
\hline 1 & 540 \\
3 & 624 \\
4 & 560 \\
5 & 546 \\
6 & 891 \\
7 & 744 \\
8 & 699 \\
9 & 1143 \\
10 & 588 \\
11 & 873 \\
12 & 1180 \\
\hline
\end{tabular}

1 In the case of dry land, half the dose of nutrients was used. In 1969/70, no dry land wheat experiments were carried out die to dry weather. 


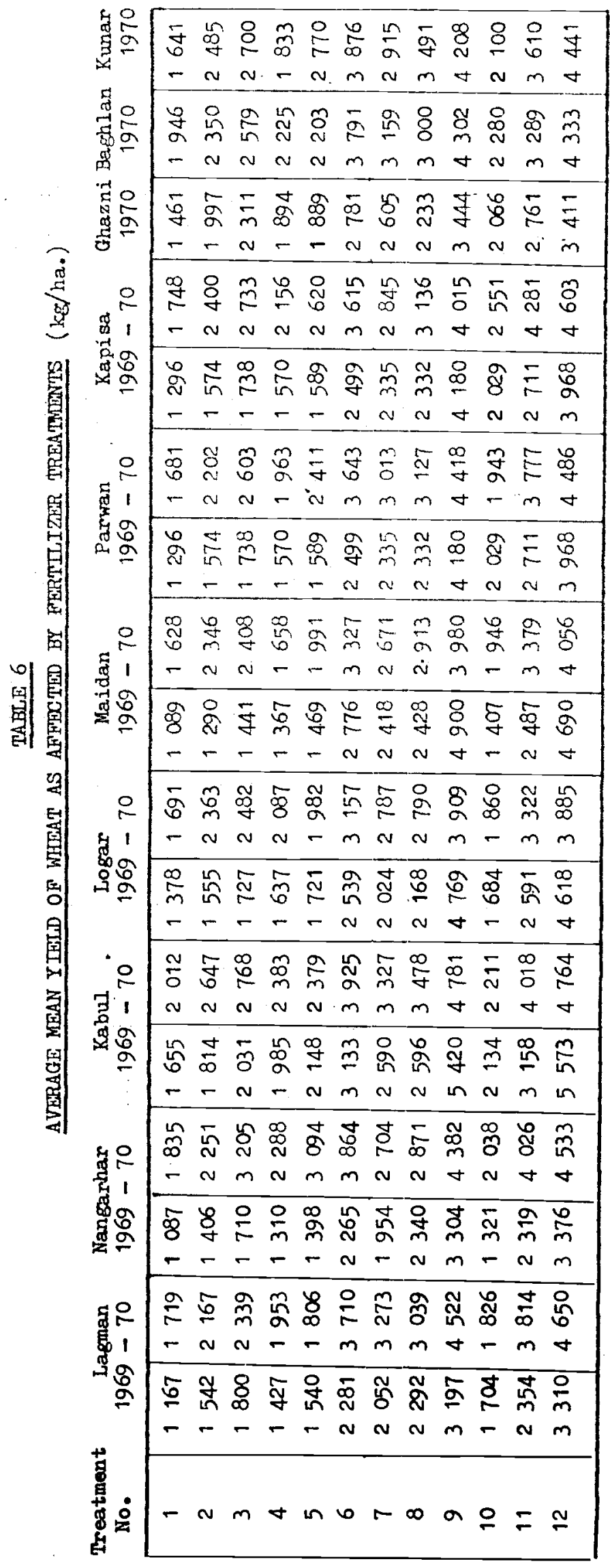



Teble 6.

In 1970-71, the same treatmentg were used as in 1969-70, and the results are given in

\subsection{Digcugsion of Rogults of Wheat Yields}

Ir 1967 to 1968 , application of $30 \mathrm{~N}+30 \mathrm{p} 205 \mathrm{~kg} / \mathrm{h}$ in the Kabul, Maican, Ghani and" Paktia areas, gave highest yields of US\$ 15.6, 1 C08, 1110 and $1035 \mathrm{~kg} / \mathrm{ha}$ respectively, and also gave highest economic return for the money invested in fertilizers. This subject is discussed more fully in Chapter 5 of this report. returns.

In the year 1968 to 1969 , application of 37 on and $37.0 \mathrm{P}_{2} \mathrm{O}_{5}$ gave the highest economic

Since the above data are only for two years, it is recommended that the experiments should be repeated for two more seagona. In the meantime, a tentative recommendation for $37 \mathrm{l}+37 \mathrm{P}_{2} \mathrm{O}_{5} \mathrm{~kg} / \mathrm{h}$ can be made for these areas.

\subsubsection{Irrigated whest}

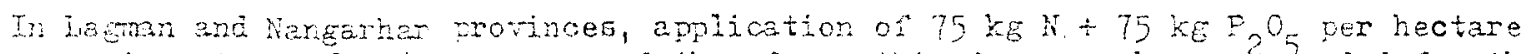
Gave economic returns for two yearn. ard therefore, this dose can be recolmended for the tameris.

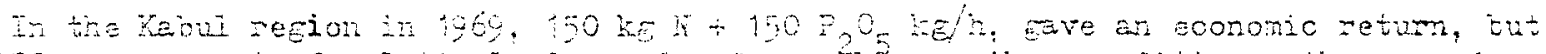

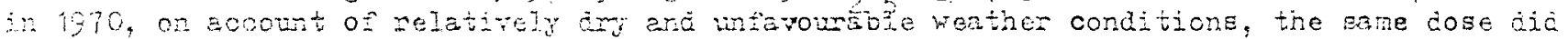
not prove economical. Instesd, $75 \mathrm{k} w+75 \mathrm{~kg} \mathrm{~F}_{2} \mathrm{O}$ gave an economic return. Therefore, it is not possible to draw conclusions. The details are ziscussed in Chapter 5.

For the provinces of Kunar, Eaghlan and Ghazni, the data are for one year only,

\subsection{Fertilizer Triais for Corn}

During 1968, about two fertilizer trials were conducted in the Logar Valley. The treatments and results are as follows:

\begin{tabular}{|c|c|c|c|c|c|}
\hline \multirow{2}{*}{$\begin{array}{l}\text { Theat } \\
\text { ment } \\
\text { No. }\end{array}$} & \multicolumn{2}{|c|}{ Treatment } & \multirow{2}{*}{$\begin{array}{c}\mathrm{kg} / \mathrm{ha} \\
\mathrm{K}_{2} \mathrm{O}\end{array}$} & \multicolumn{2}{|c|}{ Yield in $\mathrm{kg} / \mathrm{ha}$} \\
\hline & $\mathbb{N}$ & $\mathrm{P}_{2} \mathrm{O}_{5}$ & & Kinnesota 13 & Local \\
\hline 1. & 0 & 0 & 0 & 2233 & 1771 \\
\hline 2. & 40 & 0 & 0 & 2425 & 1885 \\
\hline 3. & 80 & 0 & 0 & 2760 & 2151 \\
\hline $4 \cdot$ & 0 & 40 & 0 & 2776 & 2316 \\
\hline 5. & 0 & 80 & 0 & 3084 & 2511 \\
\hline 6. & 40 & 40 & 0 & 3905 & 3225 \\
\hline 7 . & 40 & 80 & 0 & 4126 & 3548 \\
\hline 8. & 80 & 40 & 0 & 4309 & 3945 \\
\hline 9. & 80 & 80 & 0 & 6172 & 5645 \\
\hline 10. & 0 & 40 & 80 & 3890 & 3739 \\
\hline 11. & 40 & 40 & 80 & 5042 & 4. 382 \\
\hline 1?. & 80 & 80 & 80 & 6159 & 5473 \\
\hline
\end{tabular}


During the year 1969, about 71 trials were conducted with local variety. The fertilizer treatment and yielda are given below:

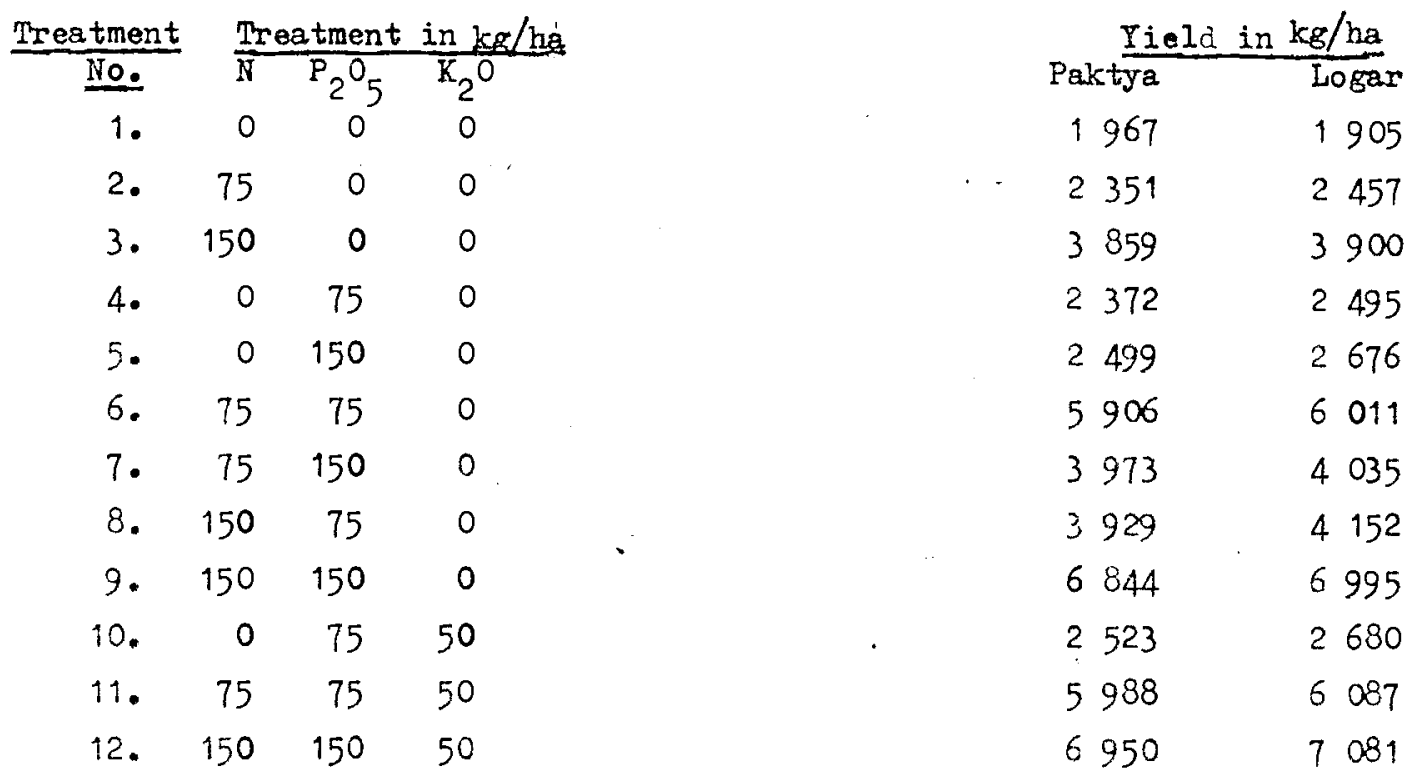

The dose of fertilizers were modified in the second year, so the data for years 1967 to 1968 , and for 1968 to 1969 , are different.

But indications show that for the provinces of Logar and Paktya, a dose of $75 \mathrm{~N}+$ $\mathrm{P}_{2} \mathrm{O}_{5} \mathrm{~kg} / \mathrm{h}$ may be economic, and therefore can be recommended tentatively for the time being.

\subsection{Experiments on Rioe in the Lagman Province}

Ten experiments for rice were conducted from 1967 to 1968 in the Lagman Province. The treatment and results are given belows

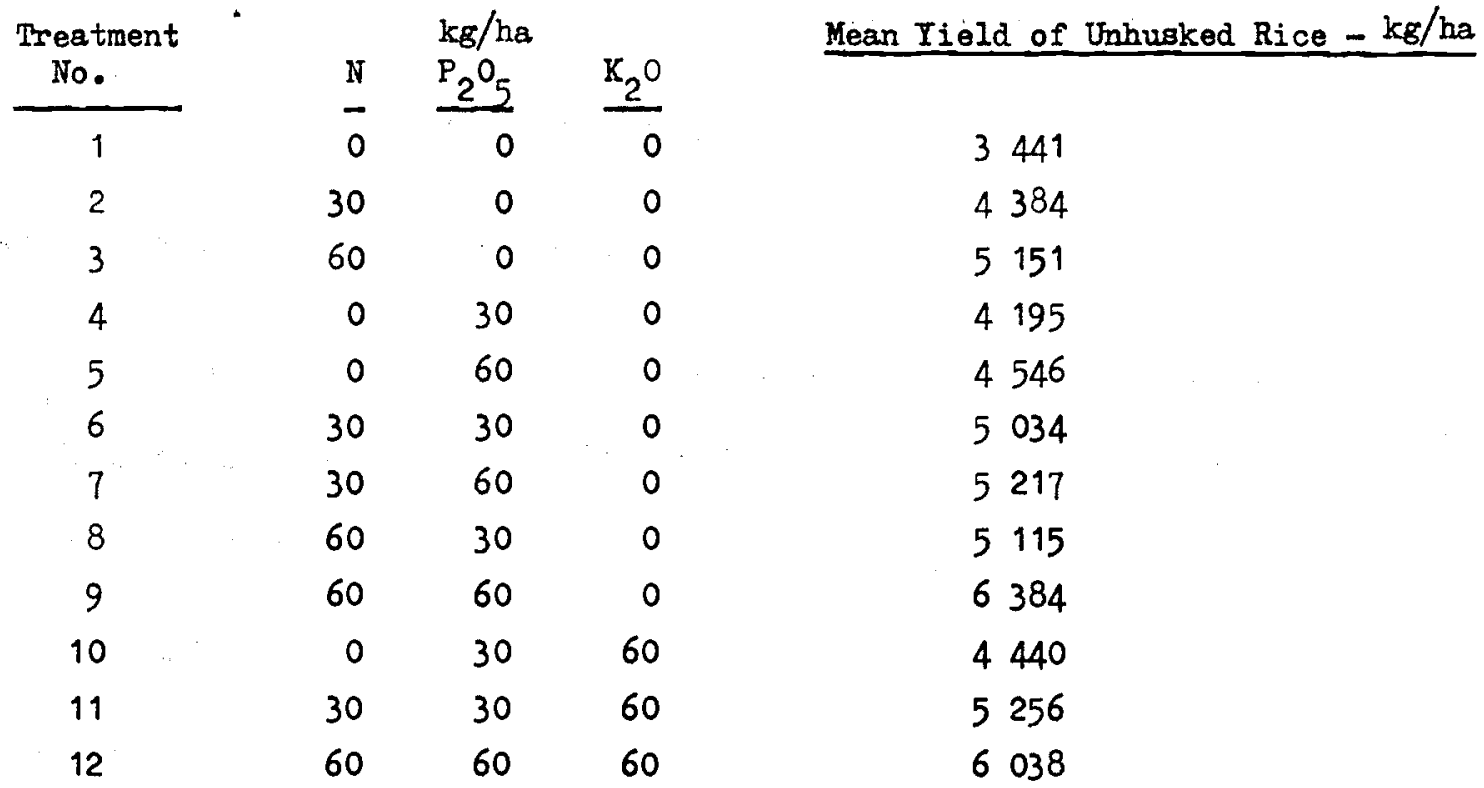

Experiments for rice were conducted for one year only, and the results indioate that application of $30 \mathrm{~N}+30 \mathrm{P}_{2} \mathrm{O}_{5} \mathrm{~kg} / \mathrm{h}$ is economical, and should be recommended for the Lagman
area. 


\subsection{Sugarbeet Fertilizer Trials}

Ten fertilizer trials on augarbet wore 21 so conducted during 1967 and 1968. The results are sumbarized belon:

\begin{tabular}{|c|c|c|c|}
\hline $\begin{array}{c}\text { Treatment } \\
\text { No. }\end{array}$ & IT & $\begin{array}{l}\mathrm{kg} / \mathrm{ha} \\
\mathrm{P}_{2} \mathrm{O}_{5}\end{array}$ & $K_{2}$ \\
\hline 1 & 0 & 0 & 0 \\
\hline 2 & 60 & 0 & 0 \\
\hline 3 & 120 & 0 & 0 \\
\hline 4 & 0 & 60 & 0 \\
\hline 5 & 0 & 120 & 0 \\
\hline 6 & 60 & 60 & 0 \\
\hline 7 & 60 & 120 & 0 \\
\hline 8 & 120 & 60 & 0 \\
\hline 9 & 120 & 120 & 0 \\
\hline 10 & 0 & $\therefore 0$ & 50 \\
\hline $1 i$ & 60 & 60 & 60 \\
\hline 12 & 120 & 120 & 60 \\
\hline
\end{tabular}

\begin{tabular}{l} 
hverage yield $\mathrm{kg} / \mathrm{ha}$ \\
\hline 22666 \\
35333 \\
33666 \\
24145 \\
25594 \\
51536 \\
51471 \\
63600 \\
70637 \\
40705 \\
53345 \\
72633
\end{tabular}

The results of one year show that $60 \mathrm{H}+60 \mathrm{P}, \mathrm{O}-\mathrm{kg} / \mathrm{h}$ is the economical dose. Soil tests were also conducted for the samples recoived fiom experimental plote, anc soil analysis deta are presented in kppendix III.

Most of the soils are low to medium in available nitrogen and phosphorus, and mediun to bigh in potassium.

A close relationship has been observed in soil test values and responses in percent of the control yield (see Appendix IV).

5. ECONOMICS OF FERTILIZER USE IN AFCHANISTAN

Average prices of crops, fertilizers and CIF prices of fertilizer material imported, are indicated below.

\begin{tabular}{|c|c|c|c|c|c|}
\hline \multicolumn{2}{|c|}{$\begin{array}{c}\text { Average Farm Prices } \\
\text { of Some Crops }\end{array}$} & \multicolumn{2}{|c|}{ Fertilizer Prices } & \multicolumn{2}{|l|}{$\begin{array}{c}\text { CIF Prices of } \\
\text { Imported Fertilizers }\end{array}$} \\
\hline \multicolumn{2}{|c|}{$\mathrm{Afa} / \mathrm{kg}$} & \multicolumn{2}{|c|}{$\mathrm{Afg} / \mathrm{kg}$} & US $\$ /$ M.T. & . \\
\hline Wheat & 6.00 & Urea & 8 & Urea from U.S.A. & 138 \\
\hline Corn & 4.25 & $\begin{array}{l}\text { Superm } \\
\text { phosphate }\end{array}$ & 3.50 & DAP from U.S.A. & 144 \\
\hline Sugarbeet & 0.60 & DAP & 9.00 & Urea from U.S.S.R. & 99 \\
\hline Rice & 4.00 & $\begin{array}{l}\text { Potsssium } \\
\text { Sulphate }\end{array}$ & 10.00 & $\begin{array}{l}\text { Super-phosphate } \\
\text { from U.S.S.R. }\end{array}$ & 40 \\
\hline
\end{tabular}


From the results of the fertilizer trials conducted in the country, the economics of fertilizer application have been worked out and presented in Tables 7, 8 and 9 for wheat; Tables 10 and 11 for com, and Tables 12 and 13 for rice and sugarbeet, respectively.

These calculations clearly indicate that by the application of correct amounts, farmer can make a net profit of 300 to 500 Afghanis for every 100 Afghanis spent on fertilizers.

The results of these experiments can also be used to project the expected increase in crop production of the country, by the application of the optimum dose of chemical fertilizer, and thus save on foreign exchange on imports of food grains. 
FABLE 7

ECONOMICS OF FER MILIZER APPLICATION - WHEAM $(1967-68)$

A. Fertilizer Response and Economic Return Under Dryland Conditions

\begin{tabular}{|c|c|c|c|c|c|c|c|}
\hline \multirow[b]{2}{*}{ Location } & \multirow{2}{*}{$\begin{array}{l}\text { Dose of } \\
\text { Nutrient } \\
\begin{array}{c}\mathrm{kg} / \mathrm{ha} \\
\mathrm{N}-\mathrm{P}\end{array}\end{array}$} & \multirow{2}{*}{\multicolumn{2}{|c|}{$\begin{array}{cl}\text { Tield in } \\
\text { Seers/J } & \mathrm{kg} / \mathrm{ha}\end{array}$}} & \multicolumn{2}{|c|}{$\begin{array}{l}\text { Increase in Yield } \\
\text { Over Control }\end{array}$} & \multicolumn{2}{|c|}{ Net Return in Afs } \\
\hline & & & & $\begin{array}{l}\text { Amount } \\
\mathrm{kg} / \mathrm{hat}\end{array}$ & Percentage & per ha & $\begin{array}{l}\text { per } 100 \text { Afs } \\
\text { Invested }\end{array}$ \\
\hline Kabul & $0-0$ & 4.7 & 167.3 & - & - & - & - \\
\hline Paiminar & $\begin{array}{r}15=0 \\
30-0 \\
0-15 \\
0-30 \\
15=15 \\
30-30\end{array}$ & $\begin{array}{r}10.9 \\
16.1 \\
0.9 \\
9.8 \\
17.4 \\
23.3\end{array}$ & $\begin{array}{r}381.6 \\
566.0 \\
240.0 \\
343.0 \\
610.0 \\
815.6\end{array}$ & $\begin{array}{r}214.3 \\
398.7 \\
72.7 \\
175.7 \\
442.7 \\
648.3\end{array}$ & $\begin{array}{r}128.0 \\
238.3 \\
43.4 \\
105.0 \\
264.5 \\
387.5\end{array}$ & $\begin{array}{r}985.8 \\
1792.1 \\
166.2 \\
514.2 \\
2086.2 \\
2743.8\end{array}$ & $\begin{array}{l}420 \\
290 \\
166.2 \\
190 \\
460 \\
340\end{array}$ \\
\hline Haiden & $0-0$ & $5 \cdot 4$ & 99.6 & - & - & - & - \\
\hline Durani & $\begin{array}{r}15-0 \\
30-0 \\
0-15 \\
0-30 \\
15-15 \\
30-30\end{array}$ & $\begin{array}{l}8.0 \\
10.0 \\
11.2 \\
13.6 \\
20.5 \\
28.8\end{array}$ & $\begin{array}{r}313 . \\
3=2.3 \\
39.0 \\
470.3 \\
718.3 \\
1003.0\end{array}$ & $\begin{array}{l}121.7 \\
160.7 \\
202.4 \\
284.7 \\
526.7 \\
816.4\end{array}$ & $\begin{array}{r}63.5 \\
83.3 \\
105.6 \\
148.5 \\
274.8 \\
426.1\end{array}$ & $\begin{array}{r}430.2 \\
364.2 \\
944.4 \\
1 \quad 168.2 \\
2590.2 \\
3785.0\end{array}$ & $\begin{array}{l}140 \\
160 \\
440 \\
310 \\
550 \\
420\end{array}$ \\
\hline Parwan & $\begin{array}{r}0-0 \\
15-0 \\
30-0 \\
60-0 \\
0-15 \\
0-30 . \\
0=60 . \\
15-15 \\
30-30 \\
60-60\end{array}$ & $\begin{array}{l}7.3 \\
13.8 \\
15.7 \\
11.9 \\
15.8 \\
16.1 \\
18.9 \\
22.6 \\
24.9 \\
31.4\end{array}$ & $\begin{array}{r}257.6 \\
484.6 \\
552.6 \\
417.0 \\
556.0 \\
566.0 \\
662.0 \\
793.0 \\
872.6 \\
1102.0 \\
\\
\text { Sprin }\end{array}$ & $\begin{array}{l}22 \overline{7} .0 \\
295.0 \\
159.4 \\
298.4 \\
308.4 \\
404.4 \\
535.4 \\
615.0 \\
844.4 \\
\text { Wheat }\end{array}$ & $\begin{array}{r}- \\
88.1 \\
114.5 \\
61.8 \\
115.8 \\
119.7 \\
156.9 \\
207.8 \\
238.7 \\
327.7\end{array}$ & $\begin{array}{lc} & - \\
1 & 062.0 \\
1 & 170.0 \\
& 243.6 \\
1 & 520.4 \\
1 & 310.4 \\
1 & 346.4 \\
2 & 642.4 \\
2 & 550.0 \\
2 & 786.4\end{array}$ & $\begin{array}{l}- \\
450 \\
290 \\
- \\
660 \\
340 \\
220 \\
560 \\
320 \\
230\end{array}$ \\
\hline Ghazni & $\begin{array}{r}0-0 \\
15-0 \\
30-0 \\
0-15 \\
0-30 \\
15-15 \\
30-30\end{array}$ & $\begin{array}{r}9.5 \\
13.1 \\
18.9 \\
9.6 \\
11.4 \\
21.3 \\
31.7\end{array}$ & $\begin{array}{r}333.8 \\
459.0 \\
663.8 \\
337.8 \\
402.5 \\
747.5 \\
1110.0\end{array}$ & $\begin{array}{r}-\overline{125.2} \\
270.0 \\
4.0 \\
68.7 \\
413.7 \\
776.2\end{array}$ & $\begin{array}{r}- \\
37.5 \\
80.0 \\
- \\
20.6 \\
122.3 \\
232.5\end{array}$ & $\begin{array}{cc} & - \\
& 451.2 \\
1 & 020.0 \\
- & \\
142.2 \\
1 & 912.2 \\
3 & 517.2\end{array}$ & $\begin{array}{l}- \\
250 \\
270 \\
- \\
150 \\
430 \\
400\end{array}$ \\
\hline Paktia & $\begin{array}{r}0-0 \\
15-0 \\
30=0 \\
0-15 \\
0-30 \\
15-15 \\
30-30\end{array}$ & $\begin{array}{r}6.7 \\
11.4 \\
16.0 \\
10.1 \\
10.0 \\
21.1 \\
29.5\end{array}$ & $\begin{array}{r}237.7 \\
402.5 \\
560.5 \\
355.5 \\
354.2 \\
740.2 \\
1035.0\end{array}$ & $\begin{array}{l}16 \overrightarrow{4} .8 \\
322.8 \\
117.8 \\
116.5 \\
502.5 \\
797.3\end{array}$ & $\begin{array}{r}- \\
69.3 \\
135.8 \\
49.5 \\
49.5 \\
211.4 \\
335.4\end{array}$ & 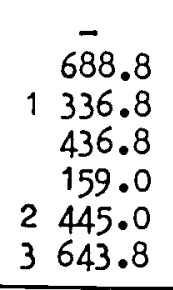 & $\begin{array}{l}- \\
320 \\
320 \\
260 \\
120 \\
520 \\
420\end{array}$ \\
\hline
\end{tabular}


Note: For calculatione, prices used are as follows:

Price in Afghanis per $\mathrm{kg} \mathrm{N}=20$

$$
\text { per } \begin{aligned}
\mathrm{k}_{B} \mathrm{P}_{2} \mathrm{O}_{5} & =18 \\
\text { wheat } & =06
\end{aligned}
$$

\begin{tabular}{|c|c|c|c|c|c|c|c|}
\hline \multirow{3}{*}{$\frac{\text { Location }}{\text { Kabul }}$} & \multirow{2}{*}{$\begin{array}{l}\text { Dose of } \\
\frac{\text { Nutrient }}{\mathrm{kg} / \mathrm{ha}} \\
\mathrm{N}-\mathrm{P}\end{array}$} & \multirow{2}{*}{\multicolumn{2}{|c|}{$\begin{array}{ll}\text { Yield in } & \text { Seers/J } \\
\text { Sg/ha }\end{array}$}} & \multirow{2}{*}{\multicolumn{2}{|c|}{$\begin{array}{l}\text { Increase in Yield } \\
\text { Over Control } \\
\text { Amount } \\
\mathrm{kg} / \mathrm{ha} \text { Percentage }\end{array}$}} & \multicolumn{2}{|c|}{ Net Return in Afs } \\
\hline & & & & & & per ha. & $\begin{array}{l}\text { fer } 100 \text { ifs } \\
\text { invested }\end{array}$ \\
\hline & $0-0$ & $13 \cdot 3$ & 468.6 & - & - & - & - \\
\hline Paiminar & $\begin{array}{r}15=0 \\
30=0 \\
60-0 \\
0=15 \\
0=30 \\
0-60 \\
5=15 \\
30=30\end{array}$ & $\begin{array}{l}16.2 \\
19.8 \\
22.0 \\
11.3 \\
13.4 \\
16.2 \\
31.9 \\
54.4\end{array}$ & $\begin{array}{r}568.0 \\
696.3 \\
773.0 \\
398.0 \\
469.0 \\
568.0 \\
1117.3 \\
1906.6\end{array}$ & $\begin{array}{r}99.4 \\
227.7 \\
304.4 \\
70.6 \\
0.4 \\
99.4 \\
684.7 \\
1437.8\end{array}$ & $\begin{array}{c}21.2 \\
48.8 \\
64.0 \\
- \\
- \\
21.2 \\
146.1 \\
307.0\end{array}$ & $\begin{array}{c}296.4 \\
766.2 \\
626.4 \\
- \\
- \\
483.6 \\
3322.2 \\
7.486 .8\end{array}$ & $\begin{array}{l}190 \\
120 \\
152 \\
- \\
- \\
\overline{682} \\
756\end{array}$ \\
\hline $\begin{array}{l}\text { Logar } \\
\text { Shikarikala }\end{array}$ & $\begin{array}{r}0-0 \\
15-0 \\
30-0 \\
60-0 \\
0-15 \\
0-30 \\
0-60 \\
15-15 \\
30=30 \\
60-60\end{array}$ & $\begin{array}{l}12.2 \\
13.0 \\
16.9 \\
19.3 \\
12.6 \\
16.5 \\
18.4 \\
50.5 \\
61.1 \\
68.2\end{array}$ & $\begin{array}{r}428.3 \\
455.3 \\
592.0 \\
675.6 \\
442.0 \\
584.0 \\
644.0 \\
1768.6 \\
2140.3 \\
2390.0\end{array}$ & $\begin{array}{r}\overline{2} \\
27.0 \\
163.7 \\
247.3 \\
13.7 \\
155.7 \\
215.7 \\
1340.3 \\
1712.0 \\
1962.0\end{array}$ & $\begin{array}{l}\overline{6} .3 \\
38.3 \\
57.7 \\
3.2 \\
36.3 \\
50.3 \\
312 \\
399.7 \\
458.0\end{array}$ & $\begin{array}{ll} & - \\
& 138 \\
382.2 \\
283.8 \\
187.8 \\
394.2 \\
\\
214 \\
7471.8 \\
9 & 132.0 \\
9 & 492.0\end{array}$ & $\begin{array}{r}- \\
\overline{160} \\
120 \\
- \\
170 \\
120 \\
1410 \\
900 \\
510\end{array}$ \\
\hline
\end{tabular}

B. Fertilizer Response and Economio Return Under Irrigated Conditions 
TABLE

ENOIOMICS OF HERTHLIZER APPLICATION - WHE $1 T$ T (1968-60)

\begin{tabular}{|c|c|c|c|c|c|c|c|}
\hline Province & Treatment & $\begin{array}{l}\text { hverage } \\
\text { yiala } \\
\mathrm{kg} / \mathrm{ha}\end{array}$ & $\begin{array}{l}\text { Increase } \\
\text { in yielò } \\
\text { over con- } \\
\text { trol } \mathrm{kg} / \mathrm{ha}\end{array}$ & $\begin{array}{l}\text { Axtra pro- } \\
\text { int due to } \\
\text { increased } \\
y \text { ield } \\
\text { Afo./ha }\end{array}$ & $\begin{array}{l}\text { Cost of } \\
\text { fertilizer } \\
\text { Afgianis } \\
\quad / h a\end{array}$ & $\begin{array}{l}\text { Net pro- } \\
\text { fit } \\
\text { Afghanis } \\
\text { /he }\end{array}$ & $\begin{array}{l}\text { Profit for } \\
\text { every } 100 \\
\text { Afghanis } \\
\text { invested }\end{array}$ \\
\hline$i$ & 2 & 3 & 4 & 5 & 6 & 7 & 8 \\
\hline \multirow[t]{12}{*}{ Lagman } & $0-0-0$ & 1167 & - & - & - & - & - \\
\hline & $75-0-$ & 1542 & 375 & 2250 & 1500 & 750 & 150.0 \\
\hline & $50-0-0$ & 1800 & $\cdot 633$ & 3798 & 3000 & 798 & 127.0 \\
\hline & $0-75-$ & 1427 & 260 & 1560 & 1350 & 210 & 116.0 \\
\hline & $0-150 \ldots$ & 1520 & 373 & 2230 & 2700 & - & - \\
\hline & $75-75-$ & 20 & $i 114$ & 6604 & 2850 & 3636 & 235.0 \\
\hline & $5-150-0$ & 205 & 834 & 5304 & 4200 & 1.04 & 125.0 \\
\hline & $150-75-0$ & 202 & $\therefore 125$ & 6750 & 4300 & 2800 & 155.0 \\
\hline & $50-150-0$ & 397 & 2030 & 12180 & 5700 & 6480 & 214.0 \\
\hline & $0-75-100$ & 1704 & 537 & 3222 & 3350 & - & - \\
\hline & $75-75-100$ & 2354 & 1187 & 7122 & 4850 & 2272 & 147.0 \\
\hline & $150-150-100$ & 3310 & 2143 & 12858 & 7700 & 5158 & 167.0 \\
\hline \multirow{12}{*}{ Nangarhar } & $=0-0-0$ & 1087 & - & - & - & - & - \\
\hline & $75-0-0$ & 1406 & 319 & 1914 & 1500 & 414 & 128 \\
\hline & $50-0-.0$ & 1710 & 623 & 3738 & 3000 & 738 & 125 \\
\hline & $0-75-0$ & 1310 & 223 & 1338 & 1350 & - & - \\
\hline & $0-150-0$ & 1398 & 311 & $\hat{1} 866$ & 2700 & - & - \\
\hline & $75-75-0$ & 2265 & 1178 & 7068 & 2850 & 4218 & 248.0 \\
\hline & $75-150-0$ & 1954 & 867 & 5202 & 4200 & 1002 & 124.0 \\
\hline & $50-75-0$ & 2340 & 1253 & 7518 & 4350 & 3168 & 173.0 \\
\hline & $150-150-0$ & 3304 & 2217 & 13302 & 5700 & 7602 & 235.0 \\
\hline & $0-75-100$ & 1321 & 234 & 1404 & 3350 & - & - \\
\hline & $75-75-100$ & 2319 & 1232 & 7392 & 4850 & 2542 & 152.0 \\
\hline & $150-150-100$ & 3376 & 2289 & 13734 & 7700 & 6.034 & 179.0 \\
\hline
\end{tabular}

Note: Cost of fertilizer $-\mathrm{N}=20, \mathrm{P}_{2} \mathrm{O}_{5}=18, \mathrm{~K}_{2} \mathrm{O}=20 \mathrm{Afs} / \mathrm{kg}$; Wheat $=6 \mathrm{Afs} . / \mathrm{kg}$. 


\begin{tabular}{|c|c|c|c|c|c|c|c|}
\hline Province & Theatment & $\begin{array}{c}\text { Average } \\
\text { yield } \\
\mathrm{kg} / \mathrm{ha}\end{array}$ & $\begin{array}{l}\text { Iner6ase } \\
\text { in jield } \\
\text { over con- } \\
\text { trol kg/ha }\end{array}$ & $\begin{array}{l}\text { Extra pro- } \\
\text { fit due to } \\
\text { increased } \\
\text { yield } \\
\text { Afs./ha }\end{array}$ & $\begin{array}{l}\text { Cost of } \\
\text { fertilizer } \\
\text { Afghanis } \\
\text { /ha }\end{array}$ & $\begin{array}{l}\text { Net pro- } \\
\text { fit } \\
\text { Afginanis } \\
\text { /ha }\end{array}$ & $\begin{array}{l}\text { Profit fur } \\
\text { every } 100 \\
\text { Afghanis } \\
\text { invested } \\
i\end{array}$ \\
\hline 1 & 2 & 3 & 4 & 5 & 6 & 7 & 8 \\
\hline Kabul & $\begin{array}{r}0-0-0 \\
75-0-0 \\
150-0-0 \\
0-75-0 \\
0-150-0 \\
75-75-0 \\
75-150-0 \\
150-75-0 \\
150-150-0 \\
0-75-100 \\
75-75-100 \\
150-150-100\end{array}$ & $\begin{array}{ll}1 & 655 \\
1 & 814 \\
2 & 031 \\
1 & 985 \\
2 & 148 \\
3 & 133 \\
2 & 590 \\
2 & 596 \\
5 & 420 \\
2 & 134 \\
3 & 158 \\
5 & 573\end{array}$ & $\begin{array}{r}- \\
159 \\
376 \\
330 \\
493 \\
1478 \\
935 \\
941 \\
3765 \\
479 \\
1503 \\
3918\end{array}$ & $\begin{array}{rl} & - \\
& 954 \\
2 & 256 \\
1 & 980 \\
2 & 958 \\
2 & 868 \\
5 & 610 \\
5 & 446 \\
22 & 590 \\
2 & 874 \\
9 & 018 \\
23 & 508\end{array}$ & $\begin{array}{ll} & - \\
1 & 500 \\
3 & 000 \\
1 & 350 \\
2 & 700 \\
2 & 850 \\
4 & 200 \\
4 & 350 \\
5 & 700 \\
3 & 350 \\
4 & 850 \\
7 & 700\end{array}$ & $\begin{array}{c}- \\
- \\
- \\
630 \\
258 \\
6018 \\
1410 \\
1096 \\
16890 \\
- \\
4168 \\
13808\end{array}$ & $\begin{array}{l}- \\
- \\
- \\
147.0 \\
110.0 \\
311.0 \\
137.0 \\
125.0 \\
396.0 \\
- \\
189.0 \\
305\end{array}$ \\
\hline $\begin{array}{l}\text { Kapisa } \\
\text { and } \\
\text { Parwan }\end{array}$ & $\begin{array}{r}0-0-0 \\
75-0-0 \\
150-0-0 \\
0-75-0 \\
0-150-0 \\
75-75^{\circ}-0 \\
75-150-0 \\
150-75-0 \\
150-150-0 \\
0-75-100 \\
75-75-100 \\
150-150-100\end{array}$ & $\begin{array}{ll}1 & 296 \\
1 & 574 \\
1 & 738 \\
1 & 570 \\
1 & 589 \\
2 & 499 \\
2 & 335 \\
2 & 332 \\
4 & 180 \\
2 & 029 \\
2 & 711 \\
3 & 968\end{array}$ & $\begin{array}{ll} & - \\
& 278 \\
& 442 \\
& 274 \\
& 293 \\
1 & 203 \\
1 & 039 \\
1 & 036 \\
2 & 884 \\
& 733 \\
1 & 415 \\
2 & 672\end{array}$ & $\begin{array}{rl} & - \\
1 & 668 \\
2 & 652 \\
1 & 644 \\
1 & 758 \\
7 & 218 \\
6 & 234 \\
6 & 216 \\
17 & 304 \\
4 & 398 \\
8 & 490 \\
16 & 032\end{array}$ & $\begin{array}{ll} & - \\
1 & 500 \\
3 & 000 \\
1 & 350 \\
2 & 700 \\
2 & 850 \\
4 & 200 \\
4 & 350 \\
5 & 700 \\
3 & 350 \\
4 & 850 \\
7 & 700\end{array}$ & $\begin{array}{ll} & - \\
& 168 \\
& - \\
& 294 \\
& - \\
4 & 368 \\
2 & 034 \\
1 & 866 \\
11 & 604 \\
1 & 048 \\
3 & 640 \\
8 & 332\end{array}$ & $\begin{array}{c}- \\
111.0 \\
- \\
122.0 \\
- \\
253.0 \\
148.0 \\
143.0 \\
304.0 \\
131.0 \\
175.0 \\
208.0\end{array}$ \\
\hline
\end{tabular}

Note: Cost of fertilizer $-\mathrm{N}=20, \mathrm{P}_{2} \mathrm{O}_{5}=18, \mathrm{~K}_{2} \mathrm{O}=20 \mathrm{Afs} . / \mathrm{kg}$; Wheat $=6 \mathrm{Afs} . / \mathrm{kg}$. 


\begin{tabular}{|c|c|c|c|c|c|c|c|}
\hline Province & Treatment & $\begin{array}{l}\text { Average } \\
\text { yield } \\
\mathrm{kg} / \mathrm{ha}\end{array}$ & $\begin{array}{l}\text { Increase } \\
\text { in yield } \\
\text { over con- } \\
\text { trol } \mathrm{kg} / \mathrm{h} / \mathrm{f}\end{array}$ & $\begin{array}{l}\text { Mxtre pro- } \\
\text { fit due to } \\
\text { increased } \\
\text { yield } \\
\text { Afs./ha }\end{array}$ & $\begin{array}{l}\text { Cost of } \\
\text { fertilizer } \\
\text { Afghanis } \\
\text { /ha }\end{array}$ & $\begin{array}{l}\text { Net pro } \\
\text { fit } \\
\text { Af ghanis } \\
\text { /ha }\end{array}$ & $\begin{array}{l}\text { Profit for } \\
\text { overy } 100 \\
\text { afghanis } \\
\text { invested }\end{array}$ \\
\hline 1 & 2 & 3 & 4 & 5 & 6 & 7 & 8 \\
\hline Logar & $\left|\begin{array}{r}0-0-0 \\
75-0-0 \\
150-0-0 \\
0-75-0 \\
0-150-0 \\
75-75-100 \\
75-150-0 \\
150-75-0 \\
150-150-0 \\
0-75-100 \\
75-75-100 \\
150-150-100\end{array}\right|$ & 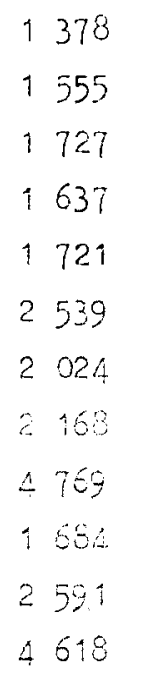 & $\begin{array}{l}- \\
177 \\
349 \\
259 \\
343 \\
116 i \\
646 \\
790 \\
339 \\
306 \\
1213 \\
3240\end{array}$ & $\begin{array}{c}- \\
1062 \\
2094 \\
1554 \\
2058 \\
6966 \\
3876 \\
4740 \\
20346 \\
1836 \\
7275 \\
19440\end{array}$ & $\begin{array}{ll} & \cdots \\
1 & 500 \\
3 & 000 \\
1 & 350 \\
2 & 700 \\
2 & 850 \\
4 & 200 \\
4 & 350 \\
5 & 700 \\
3 & 350 \\
4 & 650 \\
7 & 700\end{array}$ & 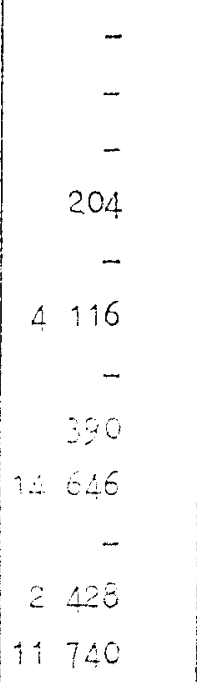 & $\begin{array}{c}- \\
- \\
- \\
115.0 \\
- \\
244.0 \\
- \\
10.0 \\
207.0 \\
- \\
150.0 \\
253.0\end{array}$ \\
\hline Maican & $\left|\begin{array}{r}0-0-0 \\
75-0-0 \\
150-0-0 \\
0-75-0 \\
0-150-0 \\
75-75-0 \\
75-150-0 \\
150-75-0 \\
150-150-0 \\
0-75-100 \\
75-75-100 \\
150-150-100\end{array}\right|$ & $\begin{array}{ll}1 & 089 \\
1 & 290 \\
1 & 441 \\
1 & 367 \\
1 & 469 \\
2 & 776 \\
2 & 418 \\
2 & 428 \\
4 & 900 \\
1 & 407 \\
2 & 487 \\
4 & 690\end{array}$ & 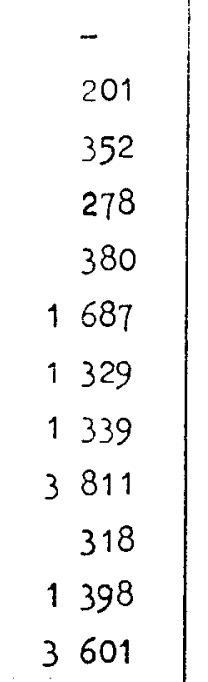 & $\begin{array}{rl} & - \\
1 & 206 \\
2 & 112 \\
1 & 668 \\
2 & 280 \\
10 & 122 \\
7 & 974 \\
8 & 034 \\
22 & 866 \\
1 & 908 \\
8 & 388 \\
21 & 606\end{array}$ & $\begin{array}{ll} & - \\
1 & 500 \\
3 & 000 \\
1 & 350 \\
2 & 700 \\
2 & 850 \\
4 & 200 \\
4 & 350 \\
5 & 700 \\
3 & 350 \\
4 & 850 \\
7 & 700\end{array}$ & 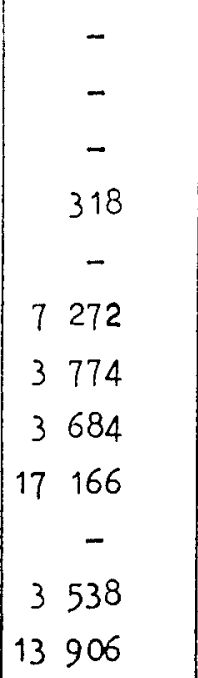 & $\begin{array}{c}- \\
- \\
- \\
124.0 \\
- \\
355.0 \\
190.0 \\
185.0 \\
401.0 \\
- \\
173.0 \\
281.0\end{array}$ \\
\hline
\end{tabular}

Note: Cost of fertilizer $-\mathrm{N}=20, \mathrm{P}_{2} \mathrm{O}_{5}=18, \mathrm{~K}_{2} \mathrm{O}=20 \mathrm{Afs} . / \mathrm{kg}$; Wheat $=6 \mathrm{Afs} . / \mathrm{kg}$. 


\begin{tabular}{|c|c|c|c|c|c|c|c|}
\hline Province & Treatment & $\begin{array}{c}\text { Average } \\
\text { yield } \\
\mathrm{kg} / \mathrm{ha}\end{array}$ & $\begin{array}{l}\text { Increase } \\
\text { in yield } \\
\text { over con- } \\
\text { trol kg/ha }\end{array}$ & $\begin{array}{l}\text { Extra pro- } \\
\text { fit due to } \\
\text { increased } \\
\text { yield } \\
\text { Afs./ha }\end{array}$ & $\begin{array}{l}\text { Cost of } \\
\text { fertilizer } \\
\text { Afghanis } \\
\text { /ha }\end{array}$ & $\begin{array}{l}\text { Net pro- } \\
\text { fit } \\
\text { Afghanis } \\
\text { /ha }\end{array}$ & $\begin{array}{l}\text { Profit for } \\
\text { every } \\
\text { Afghanis } \\
\text { invested }\end{array}$ \\
\hline 1 & 2 & 3 & 4 & 5 & 6 & 7 & 8 \\
\hline Kabul & $0-0-0$ & 440 & - & - & - & - & - \\
Dry Land & $37-0-0$ & 540 & 100 & 600 & 740 & - & - \\
& $75-0-0$ & 624 & 184 & 1104 & 1500 & - & - \\
& $0-37-0$ & 560 & 120 & 720 & 666 & 54 & 108.0 \\
& $0-75-0$ & 546 & 106 & 636 & 1350 & - & - \\
& $37-37-0$ & 891 & 451 & 2706 & 1406 & 1300 & 193.0 \\
& $37-75-0$ & 744 & 304 & 1824 & 2090 & - & - \\
& $75-37-0$ & 699 & 259 & 1554 & 2166 & - & - \\
& $75-75-0$ & 143 & 703 & 4218 & 2850 & 1368 & 148.0 \\
& $0-37-50$ & 588 & 148. & 888 & 1666 & - & - \\
& $39-37-50$ & 873 & 433 & 2598 & 2406 & 192 & 108.0 \\
$75-75-50$ & 180 & 740 & 4440 & 3850 & 590 & 115.0 \\
\hline
\end{tabular}

Note: Cost of Fertilizer $-N=20, P_{2} O_{5}=18 . K_{2} O=20 \mathrm{Afs} . / \mathrm{kg} ;$ theat $=6 \mathrm{Afs} . / \mathrm{kg}$. 
TABLE 9

EXONOMICS OF FERTIIIZER APPLICATION = WHEAT $(1969-1970)$

\begin{tabular}{|c|c|c|c|c|c|c|}
\hline Province & $\begin{array}{l}\text { Fertilizer } \\
\text { Preatment }\end{array}$ & $\begin{array}{l}\text { Increate in } \\
\text { yield } \\
\text { over oon- } \\
\text { trol in } \\
\mathrm{kg} / \mathrm{ha}\end{array}$ & $\begin{array}{l}\text { Extra Pro } \\
\text { fit due to } \\
\text { increase } \\
\text { yield in Afs. } \\
\text { /ha }\end{array}$ & $\begin{array}{l}\text { Cost of } \\
\text { Eertilizer } \\
\text { in Afs./ } \\
\text { ha }\end{array}$ & $\begin{array}{l}\text { Net Profit } \\
\text { in Afs./ } \\
\text { ha }\end{array}$ & $\begin{array}{l}\text { Profit for } \\
\text { every } 100 \\
\text { Afs. } \\
\text { invested }\end{array}$ \\
\hline 1 & 2 & 3 & 4 & 5 & 6 & 7 \\
\hline \multirow[t]{12}{*}{ Lagman } & N. $\mathrm{P}_{2} \mathrm{O}_{5} \mathrm{~K}_{2} \mathrm{O}$ & & & & & \\
\hline & $75-0-0$ & 448 & 2688 & 1500 & 1188 & 79 \\
\hline & $0-150-0$ & 620 & 3720 & 3000 & 720 & 26 \\
\hline & $0-75-0$ & 234 & i40\% & 1350 & 54 & - \\
\hline & $0-150-0$ & 87 & 522 & 2700 & - & -- \\
\hline & $75-75-0$ & 991 & 1.946 & 2850 & 006 & 39 \\
\hline & $75-150-0$ & 1534 & 9324 & $A 200$ & 5122 & $12 \vdots$ \\
\hline & $150-75-0$ & 320 & 7920 & 4356 & 3570 & $8 z$ \\
\hline & $150-150-0$ & 2803 & 16818 & 5700 & 11918 & 195 \\
\hline & $0-75-100$ & 107 & 642 & 3350 & - & - \\
\hline & $75-75-100$ & 2095 & 12570 & 4850 & 7720 & 159 \\
\hline & $150-150-100$ & 2931 & 17586 & 7700 & 9886 & 128 \\
\hline \multirow{12}{*}{ Nangarhar } & N. $\mathrm{P}_{2} \mathrm{O}_{5} \cdot \mathrm{K}_{2} \mathrm{O}$ & & & & & \\
\hline & $75-0-0$ & 416 & 2496 & 1500 & 996 & 66 \\
\hline & $0-150-0$ & 1370 & 8220 & 3000 & 5220 & 174 \\
\hline & $0-75-0$ & 453 & 2718 & 1350 & 1358 & 101 \\
\hline & $0-150-0$ & 1259 & 7554 & 2700 & 4854 & 180 \\
\hline & $75-75-0$ & 2029 & 12174 & 2850 & 9324 & 327 \\
\hline & $75-150-0$ & 817 & 5232 & 4200 & 1032 & 25 \\
\hline & $150-75-0$ & 1036 & 6216 & 4359 & 1866 & 43 \\
\hline & $150-150-0$ & 2547 & 15282 & 5700 & 9582 & 168 \\
\hline & a- 75-100 & 203 & 1218 & 3350 & - & - \\
\hline & $75-75-103$ & 2191 & 13146 & 4850 & 8296 & 171 \\
\hline & $150-150-100$ & 2703 & 16218 & 7700 & 8518 & 111 \\
\hline
\end{tabular}




\begin{tabular}{|c|c|c|c|c|c|c|}
\hline Province & $\begin{array}{l}\text { Fertilizer } \\
\text { Treatment }\end{array}$ & $\begin{array}{l}\text { Increase in } \\
\text { Yield } \\
\text { over con- } \\
\text { trol in } \\
\mathrm{kg} / \mathrm{ha}\end{array}$ & $\begin{array}{l}\text { Extra Pro- } \\
\text { fit due to } \\
\text { increase } \\
\text { Yield in Afs. } \\
\text { /ha }\end{array}$ & $\begin{array}{l}\text { Cost of } \\
\text { Fertilizer } \\
\text { in Afs. } \\
\text { /ha }\end{array}$ & $\begin{array}{l}\text { Net Profit } \\
\text { in Afs. } \\
\text { /ha }\end{array}$ & $\begin{array}{l}\text { Profit for } \\
\text { every } 1 \text { jo } \\
\text { Afs. } \\
\text { invested }\end{array}$ \\
\hline 1 & 2 & 3 & 4 & 5 & 6 & 7 \\
\hline \multirow[t]{12}{*}{ Kapisa } & N. $P_{2} O_{5} K_{2} \mathrm{O}$ & & & & & \\
\hline & $75-0-0$ & 652 & 3912 & 1500 & 2412 & 160 \\
\hline & $0-150-0$ & 985 & 5910 & 3000 & 2910 & 97 \\
\hline & $0-75-0$ & 402 & 2412 & 1350 & 1062 & 78 \\
\hline & $0-150-0$ & 872 & 5232 & 2700 & 2532 & 94 \\
\hline & $75-75-0$ & 1867 & 11202 & 2850 & 8352 & 293 \\
\hline & $75-150-0$ & $i 100$ & 6600 & 4200 & 2400 & 57 \\
\hline & $150-75-0$ & 1388 & 8328 & 4350 & 3978 & 91 \\
\hline & $150-150-0$ & 2267 & 13602 & 5700 & 7902 & 139 \\
\hline & $0-75-100$ & 803 & 4818 & 3350 & 1468 & 44 \\
\hline & $75-75-100$ & 2533 & 15198 & 4850 & 10348 & 213 \\
\hline & $150-150-100$ & 2855 & 17130 & 7700 & 9430 & 122 \\
\hline \multirow[t]{12}{*}{ Parwan } & N. $\mathrm{P}_{2} \mathrm{O}_{5} \mathrm{~K}_{2} \mathrm{O}$ & & & & & \\
\hline & $75-a-0$ & 521 & 3126 & 1500 & 1626 & 108 \\
\hline & $0-150-0$ & 922 & 5532 & 3000 & 2532 & 84 \\
\hline & $0-75-0$ & 282 & 1692 & 1350 & 342 & 25 \\
\hline & $0-150-0$ & 730 & 4380 & 2700 & 1680 & 62 \\
\hline & $75-75-0$ & 1962 & 11772 & 2850 & 8922 & 313 \\
\hline & $75-150-0$ & 1332 & 7992 & 4200 & 3792 & 90 \\
\hline & $150-75-0$ & 1446 & 8676 & 4350 & 4326 & 99 \\
\hline & $150-150-0$ & 2737 & 16422 & 5700 & 10722 & 188 \\
\hline & $0-75-100$ & 262 & 1572 & 3350 & - & - \\
\hline & $75-75-100$ & 2096 & 12576 & 4850 & 7726 & 159 \\
\hline & $150-150-100$ & 2805 & 16830 & 7700 & 9130 & 118 \\
\hline
\end{tabular}




\begin{tabular}{|c|c|c|c|c|c|c|}
\hline Province & $\begin{array}{l}\text { Fertilizer } \\
\text { Treatment }\end{array}$ & $\begin{array}{l}\text { Inorcase in } \\
\text { yield } \\
\text { over con- } \\
\text { trol in } \\
\mathrm{kg} / \mathrm{h}\end{array}$ & $\begin{array}{l}\text { Extra Pro-- } \\
\text { fit due to } \\
\text { incroase } \\
\text { yield in Afs } \\
\text { /hs }\end{array}$ & $\left\{\begin{array}{l}\text { Cost of } \\
\text { Fertilizer } \\
\text { in Afs. } \\
\text { /ha }\end{array}\right.$ & $\begin{array}{l}\text { Net Profit } \\
\text { in AfB. } \\
\text { /ha }\end{array}$ & $\begin{array}{l}\text { Frofit for } \\
\text { every } 100 \\
\text { Afs. } \\
\text { invested }\end{array}$ \\
\hline 1 & 2 & 3 & 4 & 5 & 6 & 7 \\
\hline \multirow[t]{12}{*}{ Kunar } & i. $\mathrm{P}_{2} \mathrm{O}_{5} \mathrm{~K}_{2} \mathrm{O}$ & & & & & \\
\hline & $75-0$ & 8.14 & 5064 & 1500 & 3564 & $2: 3$ \\
\hline & $0-150-0$ & 1059 & 6354 & 3000 & 3354 & 112 \\
\hline & $0-75-0$ & 192 & 1152 & 1350 & - & - \\
\hline & $0-150-0$ & 1129. & 6774 & 2700 & 4074 & 150 \\
\hline & $75-75-0$ & $223 \overline{3}$ & 13410 & 2350 & 10560 & 371 \\
\hline & $75-150-0$ & $\div 274$ & 7644 & 4200 & 314 & 82 \\
\hline & $150-75-0$ & 1850 & $1+100$ & 2250 & 6750 & 55 \\
\hline & $150-15000$ & 2567 & 15402 & 5700 & 9702 & 90 \\
\hline & $0-75-100$ & 459 & 3204 & 3350 & - & - \\
\hline & $75-75-100$ & 1969 & 论 814 & 4350 & 6964 & $1 \%$ \\
\hline & $150-150-100$ & 2800 & 16600 & 7700 & 9100 & 118 \\
\hline \multirow[t]{12}{*}{ KabuI } & N. $\mathrm{P}_{2} \mathrm{O}_{5} \mathrm{~K}_{2} \mathrm{O}$ & & & & & \\
\hline & $75-0-0$ & 635 & 3810 & 1500 & 2310 & .155 \\
\hline & $0-150-0$ & 756 & 4536 & 3000 & 1536 & 84 \\
\hline & $0-75-.0$ & 371 & 2226 & 1350 & 876 & 65 \\
\hline & $0-150 \cdot 0$ & 367 & 2202 & 2700 & - & - \\
\hline & $75-75-0$ & 1913 & 11478 & 2850 & 8628 & 302 \\
\hline & $75-150-0$ & 1315 & 7890 & 4200 & 3690 & 87 \\
\hline & $150-75-0$ & 1. 466 & 8796 & 4350 & 4446 & 102 \\
\hline & $15 a-15 a-0$ & 2769 & 16614 & 5700 & 10914 & 191 \\
\hline & $a-75-100$ & 199 & 1194 & 3350 & - & - \\
\hline & $75-75-100$ & 2006 & 12036 & 4850 & 7186 & 148 \\
\hline & $150-150-100$ & 2752 & 16512 & 7700 & 8812 & 114 \\
\hline
\end{tabular}




\begin{tabular}{|c|c|c|c|c|c|c|}
\hline Province & $\begin{array}{l}\text { Fertilizer } \\
\text { Treatment }\end{array}$ & $\begin{array}{l}\text { Inorease in } \\
\text { yield. } \\
\text { over oon- } \\
\text { trol in } \\
\mathrm{kg} / \mathrm{ha}\end{array}$ & $\begin{array}{l}\text { Fxtra Pro- } \\
\text { fit due to } \\
\text { increase } \\
\text { yield in Afs } \\
\quad / h a\end{array}$ & $\begin{array}{l}\text { Cost of } \\
\text { Fertilizer } \\
\text { in Afs. } \\
\text { /ha }\end{array}$ & $\begin{array}{c}\text { Net Profit } \\
\text { in Afs. } \\
/ \mathrm{ha}\end{array}$ & $\begin{array}{l}\text { Profit for } \\
\text { every } 100 \\
\text { Afs. } \\
\text { invested } \\
\end{array}$ \\
\hline 1 & 2 & 3 & 4 & 5 & 6 & 7 \\
\hline \multirow[t]{12}{*}{ Maidan } & N. $\mathrm{P}_{2} \mathrm{O}_{5} \mathrm{~K}_{2} \mathrm{O}$ & & & & & \\
\hline & $75-a$ & 718 & 4308 & 1500 & 2808 & 187 \\
\hline & $0-150-0$ & 780 & 4680 & 3000 & 1680 & 56 \\
\hline & $0-75-0$ & 30 & 180 & 1350 & - & - \\
\hline & $0-150-0$ & 363 & 2178 & 2700 & - & - \\
\hline & $75-75-0$ & 1699 & 10194 & 2850 & 7344 & 258 \\
\hline & $75-150-0$ & 1043 & 6258 & 4200 & 2058 & 49 \\
\hline & $150-75-0$ & 1285 & 7710 & 4350 & 3360 & 77 \\
\hline & $150-150-0$ & 2352 & 14112 & 5700 & 8412 & 147 \\
\hline & a $75-100$ & 328 & 1968 & 3350 & - & - \\
\hline & $75-75-100$ & 1751 & 10506 & 4850 & 5656 & 117 \\
\hline & $150-150-100$ & 2728 & 14568 & 7700 & 6868 & 89 \\
\hline \multirow[t]{12}{*}{ Iogar } & N. $\mathrm{P}_{2} \mathrm{O}_{5} \mathrm{~K}_{2} \mathrm{O}$ & & & & & \\
\hline & $75-0-0$ & 672 & 4032 & 1500 & 2532 & 168 \\
\hline & $0-150-0$ & 791 & 4746 & 3000 & 17.76 & 58 \\
\hline & $0-75-0$ & 396 & 2376 & 1350 & 1026 & 76 \\
\hline & $0-150-0$ & 296 & 1776 & 2700 & - & - \\
\hline & $75-75-0$ & 1466 & 8796 & 2850 & 5946 & 209 \\
\hline & $75-150-0$ & 1096 & 6576 & 4200 & 2376 & 59 \\
\hline & $150-75-0$ & 1099 & 6594 & 4350 & 2244 & 52 \\
\hline & $150-150-0$ & 2218 & 13308 & 5700 & $76 n 8$ & 133 \\
\hline & a $75-100$ & 169 & 1014 & 3350 & - & - \\
\hline & $75-75-100$ & 1631 & 9786 & 4850 & 3136 & 65 \\
\hline & $150-150-100$ & 2194 & 13164 & 7700 & 5464 & 71 \\
\hline
\end{tabular}




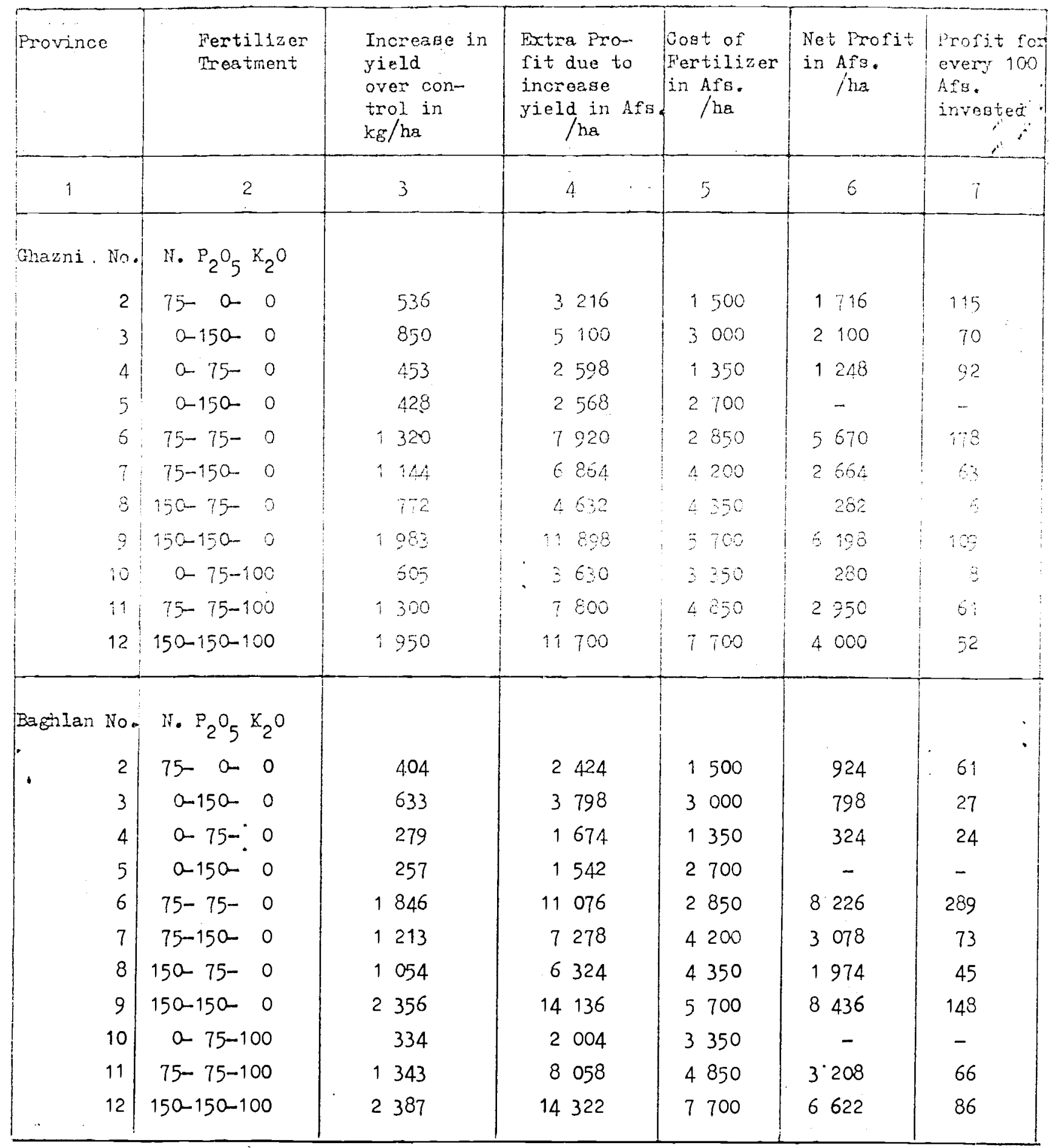

Note: Costs:- $N=20 \mathrm{Afs} / \mathrm{kg}$

$$
\begin{aligned}
& \mathrm{P}_{2} \mathrm{O}_{5}=18 \mathrm{Afs} / \mathrm{kg} \\
& \mathrm{K}_{2} \mathrm{O}=20 \mathrm{Afs} / \mathrm{kg} \\
& \text { Wheat }=6 \mathrm{Afs} / \mathrm{kg}
\end{aligned}
$$


TABLE 10

BCONOMICS OF FERTIIIZER APPLICATTON - CORN (1968-1969)

\begin{tabular}{|c|c|c|c|c|c|c|c|c|c|}
\hline \multicolumn{3}{|c|}{$\begin{array}{l}\text { Treatment } \\
\mathrm{N} \mathrm{P}_{2} \mathrm{O}_{5} \quad \mathrm{x}_{2} \mathrm{O}\end{array}$} & \multicolumn{2}{|c|}{$\begin{array}{l}\text { Average jield } \\
\mathrm{kg} / \mathrm{ha} \text { Seer } / \mathrm{j} .\end{array}$} & \multirow{2}{*}{$\begin{array}{l}\text { Increase } \\
\text { over con- } \\
\text { trol } \\
\mathrm{kg} / \mathrm{hg} \\
-\end{array}$} & \multirow{2}{*}{$\begin{array}{c}\text { Pex } \\
\text { cent }\end{array}$} & \multirow{2}{*}{$\begin{array}{l}\text { Cost of } \\
\text { Fertilizer } \\
\text { in Afs } \\
\text { /ha } \\
\end{array}$} & \multirow{2}{*}{$\begin{array}{l}\text { Net return } \\
\text { in Afs } \\
/ \mathrm{ha}\end{array}$} & $\begin{array}{l}\text { Gain or loss } \\
\text { per } 100 \mathrm{Afs} \\
\text { invested }\end{array}$ \\
\hline 1. 0 & 0 & 0 & 2233 & 63.8 & & & & & - \\
\hline 2. 40 & 0 & 0 & 2425 & 69.2 & 192 & 8.5 & 800 & 16 & + \\
\hline 3. 80 & 0 & 0 & 2760 & 78.8 & 527 & 23.6 & 1600 & 639.75 & 40 \\
\hline 4. 0 & 40 & 0 & 2776 & 79.3 & 543 & $24 \cdot 3$ & 720 & 1597.75 & +220.50 \\
\hline 5. 0 & 80 & 0 & 3084 & 88.1 & 851 & 381 & 1440 & 2176.75 & +157.1 \\
\hline 6. 40 & 40 & 0 & 3905 & 111.5 & 1672 & 74.8 & 1520 & 5586.00 & +367.50 \\
\hline 7.40 & 80 & 0 & 4126 & 117.8 & 1893 & 84.6 & 2240 & 5805.25 & +259.3 \\
\hline 8. 80 & 40 & 0 & 4309 & 123.1 & 2076 & 92.9 & 2320 & 6503.00 & +290.4 \\
\hline 9.80 & 80 & 0 & 6172 & 176.3 & 3939 & 176.4 & 3040 & 13700.75 & +450.6 \\
\hline 10. 0 & 40 & 80 & 3890 & 111.1 & 1657 & 74.3 & 2320 & 4722.25 & +203.5 \\
\hline 11. 40 & 40 & 80 & 5042 & 144.0 & 2809 & 125.7 & 3120 & 8818.25 & +282.6 \\
\hline 12.80 & 80 & 80 & 6159 & 175.9 & 3926 & 175.8 & 4640 & 11093 & +239.0 \\
\hline
\end{tabular}

Note: For above calculations prices used are as follows:

$$
\begin{aligned}
& 1 \mathrm{kgN}=20 \mathrm{AfB} \text {. } \\
& 1 \mathrm{kgP}_{2} \mathrm{O}_{5}=20 \mathrm{Afs} \text {. } \\
& 1 \mathrm{~kg} \mathrm{~K} \mathrm{~K}_{2} \mathrm{O}=20 \mathrm{Afs} \text {. } \\
& 1 \mathrm{~kg} \text { Corn }=4.25 \mathrm{Afs} \text {. }
\end{aligned}
$$




\begin{tabular}{|c|c|c|c|c|c|c|c|c|c|}
\hline $\mathrm{N} \quad \mathrm{P}_{2} \mathrm{O}_{5}$ & $\mathrm{~K}_{2} \mathrm{O}$ & & $\begin{array}{l}\text { Averag } \\
\text { ke/hat }\end{array}$ & $\begin{array}{l}\text { Seer/jold } \\
\text { seer }\end{array}$ & $\begin{array}{l}\text { Increase } \\
\text { in view } \\
\text { over control } \\
\mathrm{kg} / \mathrm{ha}\end{array}$ & $\begin{array}{l}\text { Per- } \\
\text { cent }\end{array}$ & $\begin{array}{l}\text { Cost of } \\
\text { Fertilizer } \\
\text { in Afs } \\
\text { /ha }\end{array}$ & $\begin{array}{l}\text { Net retum } \\
\text { in Afs } \\
\text { / ha }\end{array}$ & $\begin{array}{l}\text { Gain or loss } \\
\text { per } 100 \text { Afs } \\
\text { invested }\end{array}$ \\
\hline 0 & 0 & 0 & 1771 & 50.6 & - & - & - & - & - \\
\hline 2. 40 & 0 & 0 & 1885 & 53.8 & 114 & 6.4 & 800 & -315 & -39 \\
\hline 3. 80 & 0 & 0 & 2151 & 6.4 & 380 & 21.4 & 1600 & +15 & +1.0 \\
\hline 4. 0 & 40 & 0 & 2316 & 66.1 & 545 & 30.7 & 720 & 1596 & +221.6 \\
\hline 5.0 & 80 & 0 & $25 \quad 11: 6 \mid$ & $7 i .7$ & 740 & 41.7 & 1440 & 1805 & +125.3 \\
\hline 6. 40 & 40 & 0 & 3225 & 92.1 & 1454 & 82.1 & 15.20 & 4059 & +267.0 \\
\hline 7. 40 & 80 & 80 & 3548 & 101.3 & 1777 & 100.3 & 2240 & $53: 2$ & +237.1 \\
\hline 8. 80 & 40 & 0 & 3945 & 112.7 & 2174 & 122.7 & 2320 & 6919 & 4298.0 \\
\hline 9.80 & 80 & 0 & 5645 & 161.2 & 3874 & 218.7 & 3000 & 13424 & $+44: 5$ \\
\hline 10. & 40 & 80 & 3739 & 105.8 & $\div 968$ & 111.1 & 2320 & 6044 & +255.6 \\
\hline ii. 40 & 40 & 40 & 4332 & 125.2 & 2611 & 1.47 .2 & 3120 & 7976 & +655.6 \\
\hline 12. 80 & 80 & 80 & 5473 & 156.3 & 3702 & 200.0 & 4640 & 11093 & +239.0 \\
\hline
\end{tabular}

Note: For above calculations pricesused are as follows:

$$
\begin{aligned}
& 1 \mathrm{~kg}=20 \mathrm{Afs} \\
& 1 \mathrm{kgP}_{2} \mathrm{O}_{5}=18 \mathrm{Afs} \\
& \mathrm{K}_{2} \mathrm{O} \div 20 \mathrm{Afs}
\end{aligned}
$$

$1 \mathrm{~kg}$ Come 4.25 Afs. 
TABLE 11

ECONOMICS OF FERTILIZER APPLICATION - CORN (1969-1970)

Paktya

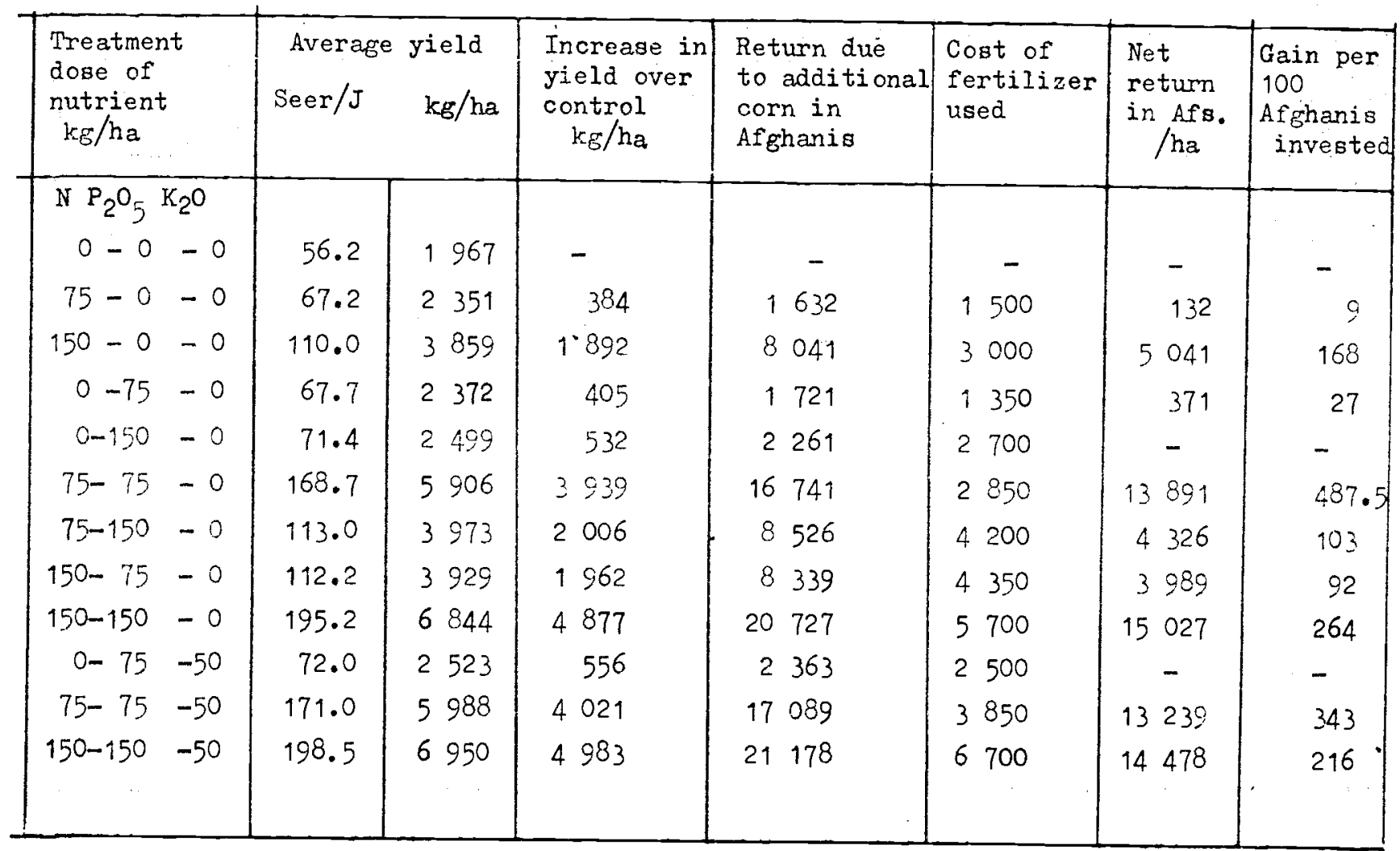

Price in Afghanis per $\mathrm{kg}$

$$
\begin{aligned}
\mathrm{N} & =20 \\
\mathrm{P}_{2} \mathrm{O}_{5} & =18 \\
\mathrm{~K}_{2} \mathrm{O} & =20 \\
\text { Corn } & =4.25
\end{aligned}
$$


Logar

\begin{tabular}{|c|c|c|c|c|c|c|c|}
\hline $\begin{array}{l}\text { Theatment } \\
\text { dose of } \\
\text { nutrient } \\
\mathrm{kg} / \mathrm{ha}\end{array}$ & $\begin{array}{l}\text { Averae } \\
\text { Seer/J }\end{array}$ & $\begin{array}{l}\text { yield } \\
\mathrm{kg} / \mathrm{ha}\end{array}$ & $\begin{array}{l}\text { Increase in } \\
\text { yield over } \\
\text { control } \\
\mathrm{kg} / \mathrm{ha}\end{array}$ & $\begin{array}{l}\text { Retum due } \\
\text { to additional } \\
\text { com in } \\
\text { Afghania }\end{array}$ & $\begin{array}{l}\text { Cost of } \\
\text { fertilizen } \\
\text { used }\end{array}$ & $\begin{array}{l}\text { Net } \\
\text { return } \\
\text { in Afs. } \\
\text { /ha }\end{array}$ & $\begin{array}{l}\text { Gain } \\
\text { per } 100 \\
\text { Afghanis } \\
\text { invested }\end{array}$ \\
\hline $\mathrm{NP}_{2} \mathrm{O}_{5} \cdot \mathrm{K}_{2} \mathrm{O}$ & & & & & & & \\
\hline $0-0-0$ & $54 \cdot 4$ & 1905 & - & - & - & - & - \\
\hline $75-0-0$ & 70.4 & 2457 & 552 & 2346 & 1500 & 846 & 56.4 \\
\hline $150-0-0$ & 111.4 & 3900 & 1995 & 8479 & 3000 & 5479 & $149 \cdot 3$ \\
\hline $0-75-0$ & 71.2 & 2495 & 590 & 2508 & 1350 & 1158 & 85.7 \\
\hline $0-150-0$ & 76.4 & 2676 & 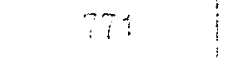 & 3277 & 2700 & $5 \%$ & $2+3$ \\
\hline $75-75-0$ & 171.7 & 6013 & 4706 & i7 450 & $\therefore 630$ & $1 \div 600$ & $51 \%$. \\
\hline $75-150-0$ & 115.2 & 4025 & 2130 & 8052 & $\therefore 200$ & $\therefore 252$ & $45: 5$ \\
\hline $150-75-0$ & 118.6 & 4152 & 2247 & 950 & $\therefore 350$ & 5200 & $119=$ \\
\hline $150-150-0$ & 199.8 & 6995 & 5000 & 21632 & 5700 & 15932 & 279.5 \\
\hline $0-75-50$ & 76.5 & $? 680$ & 775 & 3204 & 2500 & 794 & 32.0 \\
\hline $75-75-50$ & 173.9 & 6087 & 4182 & 17774 & 3850 & 13.924 & 261.6 \\
\hline $150-150-50$ & 202.3 & 7081 & 5176 & 21998 & 6700 & 15298 & 228.3. \\
\hline
\end{tabular}

Price in Afghanis per $\mathrm{kg}$

$$
\begin{aligned}
\mathrm{N} & =20 \\
\mathrm{P}_{2} \mathrm{O}_{5} & =18 \\
\mathrm{~K}_{2} \mathrm{O} & =20 \\
\text { Corn } & =4.25
\end{aligned}
$$

From the data of economic return, it is clear that the most economical dose is $75 \mathrm{~N}+$ $75 \mathrm{P}_{2} \mathrm{O}_{5} \mathrm{~kg} / \mathrm{h}$. For every 100 Afghanis invested in the fertilizer, the farmer would make a net profit of 487 and 512 Afghanis in Paktya and Logar provinces respectively. 
TABLE 12

ECONOMICS OF FERTILIZER APPLICATION - RICE (1967-1968)

\begin{tabular}{|c|c|c|c|c|c|c|c|c|c|c|}
\hline $\begin{array}{l}\text { Treat- } \\
\text { ment } \\
\text { No. }\end{array}$ & N P & ${ }_{2} \mathrm{O}_{5} \mathrm{~K}$ & & $\begin{array}{l}\text { Average } \\
\text { Seer/J }\end{array}$ & $\begin{array}{l}\text { yield } \\
\mathrm{kg} / \mathrm{ha}\end{array}$ & $\begin{array}{l}\text { Increase in } \\
\text { yield over } \\
\text { control } \\
\mathrm{kg} / \mathrm{ha}\end{array}$ & $\begin{array}{l}\text { Gain due } \\
\text { to incr- } \\
\text { ease over } \\
\text { control }\end{array}$ & $\begin{array}{l}\text { Cost of } \\
\text { fertilizer } \\
\text { Afs. }\end{array}$ & $\begin{array}{l}\text { Net return } \\
\text { in } \\
\text { Afs./ha }\end{array}$ & $\begin{array}{l}\text { Gain per } \\
100 \text { Afs } \\
\text { invested } \\
\text { Afs }\end{array}$ \\
\hline 1 & 0 & -0 & -0 & 98.3 & 3441 & - & - & - & - & - \\
\hline 2 & 30 & -0 & -0 & 125.2 & 4384 & 943 & 3772 & 600 & 3172 & 528 \\
\hline 3 & 60 & -0 & - & 147.1 & 5151 & 1710 & 6840 & 1200 & 5640 & 470 \\
\hline 4 & 0 & -30 & -0 & 119.8 & 4195 & 754 & 3016 & 540 & 2476 & 458 \\
\hline 5 & 0 & -60 & -0 & 129.8 & 4546 & 1105 & 4420 & 1080 & 3340 & 300 \\
\hline 6 & 30 & -30 & -0 & 143.8 & 5034 & 1593 & 6372 & 1140 & 5232 & 458 \\
\hline 7 & 30 & -60 & -0 & 149.0 & 5217 & 1776 & 7104 & 1680 & 5424 & 323 \\
\hline 8 & 60 & -30 & -0 & 146.1 & 5115 & 1674 & 6696 & 1740 & 4956 & 284 \\
\hline 9 & 60 & -60 & -0 & 182.4 & 6384 & 2943 & 11772 & 2280 & 9492 & 416 \\
\hline 10 & 0 & -30 & -60 & 126.9 & 4440 & 999 & 3996 & 1740 & 2256 & 129 \\
\hline 11 & 30 & -30 & -60 & 150.2 & 5256 & 1815 & 7260 & 2340 & 4920 & 210 \\
\hline 12 & 60 & -60 & -60 & 172.5 & 6038 & 2597 & 10388 & 3480 & 6908 & 198 \\
\hline
\end{tabular}

Note: For these calculations, the following prices were used:

Price per kg :

$$
\begin{aligned}
\mathrm{N} & =20 \mathrm{Afs} \\
\mathrm{P}_{2} \mathrm{O}_{5} & =18 \\
\mathrm{~K}_{2} \mathrm{O} & =20 \\
\text { Unhusked rice } & =4
\end{aligned}
$$


TABLE 13

ECONOHICS OP FERTILIZER APPLICATION - SURAREEET (1967-1968)

\begin{tabular}{|c|c|c|c|c|c|c|c|c|c|c|}
\hline $\begin{array}{l}\text { Treat- } \\
\text { ment } \\
\text { No. }\end{array}$ & in & $2^{0} 5^{K}$ & $2^{0}$ & $\begin{array}{l}\text { Average } \\
\text { Seer/J }\end{array}$ & $\begin{array}{l}\text { yielda } \\
\mathrm{kg}_{\mathrm{B}} / \mathrm{ha}\end{array}$ & $\begin{array}{c}\text { Increase } \\
\text { in over } \\
\text { control } \\
\mathrm{kg} / \mathrm{ha}\end{array}$ & $\begin{array}{l}\text { Gain due } \\
\text { to in- } \\
\text { crease in } \\
\text { yield afs }\end{array}$ & $\begin{array}{l}\text { Cost of } \\
\text { fertilizer } \\
\text { Afs. }\end{array}$ & $\begin{array}{l}\text { Net return } \\
\text { in Afs. } \\
/ \text { hs }\end{array}$ & $\begin{array}{l}\text { Gain or } \\
\text { loss in } \\
\text { Afs per } \\
\text { ioo } \\
\text { Afs in- } \\
\text { vested }\end{array}$ \\
\hline$i$ & 0 & -0 & -0 & 647.6 & 22666 & - & - & - & - & - \\
\hline 2 & 60 & -0 & -0 & 1009.5 & 353.33 & 12667 & 7600 & 1200 & 6400 & 530 \\
\hline 3 & $4 \geq 0$ & -0 & - & 1104.7 & 38666 & 16000 & 9600 & 2400 & 7200 & 300 \\
\hline 4 & 0 & -60 & -0 & 680.8 & $24 \quad 145$ & 1479 & 897 & 1080 & $10:$ & $\cdots$ \\
\hline 5 & $o$ & -120 & -0 & 731.2 & $25 \quad 594$ & 220 & 175 & 2160 & $40:$ & $\cdots$ \\
\hline 6 & 60 & -60 & -0 & 1472.4 & $5+536$ & 2067 & 798 & 2280 & 15042 & 65 \\
\hline$i$ & 60 & -120 & -0 & 1470.6 & 5441 & 28005 & 17263 & 3300 & 13923 & 210 \\
\hline 8 & $h=0$ & -60 & -0 & 1817.1 & 63600 & 40943 & 24566 & 3480 & 21086 & 620 \\
\hline 9 & 120 & -120 & -0 & 2018.2 & 70637 & 47971 & 23783 & 4560 & 24223 & 520 \\
\hline 10 & 0 & -60 & -60 & 1165.2 & 40785 & 18119 & 10871 & 2400 & 8471 & 350 \\
\hline 11 & 60 & -60 & -60 & 1667.0 & 58346 & 35680 & 21408 & 3480 & 17928 & 510 \\
\hline 12 & 120 & -120 & -60 & 2075.2 & 72633 & 49967 & 29980 & 5760 & 24220 & 420 \\
\hline
\end{tabular}

Note: For these calculations, the following prices were used:

Price in Afghanis per kg:

$$
\begin{aligned}
N & =20 \\
\mathrm{P}_{2} \mathrm{O}_{5} & =18 \\
\text { Sugarbeet } & =0.6
\end{aligned}
$$




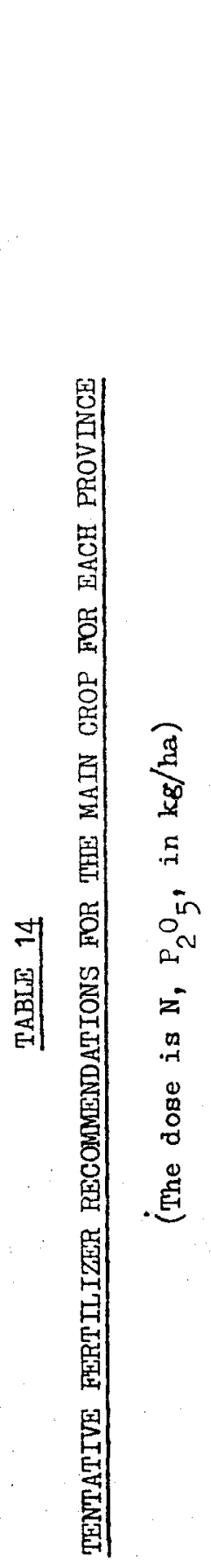

\begin{tabular}{|c|c|c|c|c|}
\hline 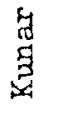 & $\stackrel{n}{\stackrel{n}{\mathfrak{n}}}$ & 1 & 1 & 1 \\
\hline 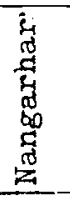 & $\stackrel{n}{\frac{n}{1}}$ & 1 & - & 1 \\
\hline 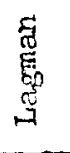 & $\begin{array}{l}\frac{9}{n} \\
\frac{1}{1} \\
\frac{1}{2}\end{array}$ & 1 & 유 & 1 \\
\hline 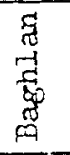 & $\underset{\substack{n \\
\stackrel{n}{n}}}{\stackrel{n}{r}}$ & 1 & 1 & $\begin{array}{l}8 \\
8 \\
8\end{array}$ \\
\hline 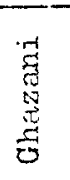 & $\stackrel{5}{m} \stackrel{\substack{n \\
m}}{1}$ & 1 & 1 & 1 \\
\hline 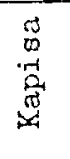 & $\frac{0}{1}$ & 1 & 1 & 1 \\
\hline 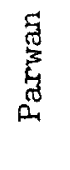 & 옴 & 1 & $\mathbf{I}$ & 1 \\
\hline 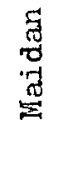 & $\frac{}{i n}$ & 1 & 1 & 1 \\
\hline 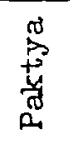 & $\frac{\hat{n}}{m}$, & $\frac{n}{n}$ & $\mathbf{I}$ & 1 \\
\hline 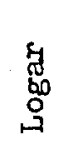 & 윰 & $\stackrel{n}{n}$ & 1 & 1 \\
\hline 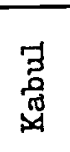 & $\begin{array}{ll}\stackrel{n}{m} & \stackrel{\circ}{n} \\
\frac{1}{m} & \stackrel{d}{n}\end{array}$ & 1 & 1 & 1 \\
\hline 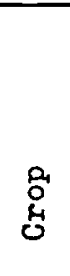 & 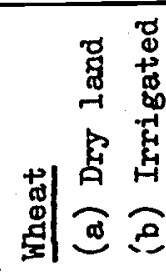 & 뎡 & $\begin{array}{l}\text { : } \\
\text { : } \\
\text { | }\end{array}$ & $\begin{array}{l}0 \\
0 \\
0 \\
\vdots \\
\vdots \\
0 \\
0 \\
0\end{array}$ \\
\hline
\end{tabular}




\title{
RECOMENDATIONS
}

\subsection{Future Outline of hork}

It is strongly recominended that a project on Soil fertility and Fertilizer Use Development in Afehanistan be initiated.

A suggested description of the activities of the project is given belor:

6.1 .1 General

a) The project should have a duration of five years, and should be carried out in co-operation with the Ministry of Agriculture and Irrifation, with the participation of other divisions and organiationg, such as:

\author{
Soils Department \\ - Faculty of Agriculture \\ Bxtension Department
}

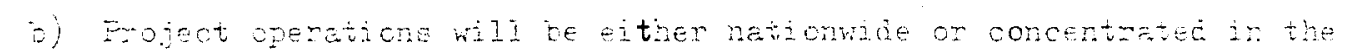

selected aneze.

c) Dat: on the response of crope to fentilizer and verious asioulura: practices that are of importance to project activities, should be aseeribled and anelysec if possible according to soil groups and Eeological conditions, to form a basiffor the pleming of the experimental work.

\subsection{Field Programmes to Promote Fertilizer Use}

Fertilizer trials will be carried out on farmers' fields. The responses to nitrogen, phosphorus and potash will be recorded in terms of yield increases and economic effects. The efficiency of different forms of fertilizer materials will be compared. The best time and method of fertilizer application; interaction of fertilizer with organic manuring; improved and high yielding varieties; cultural practices and plant protection measures will be investigated. Relationship between soil type, soil conditions, drainage, aeration and fertilizer responses will be established.

Fertilizer demonstrations will be carried on farmers' fields in order to show the farmers the effects of fertilizers, and to advise them how to apply fertilizers correctly. Contests between farmers for the highest yields (on a village, province and country basis), should be held in order to show them the importance of the use of fertilizers. These activities will be carried out in co-operation with the extension service after special training on fertilizer use extension work has been given to the extension staff.

For establishing a soil testing service, the most appropriate methods for routine analysis will be tested and selected. A central and several regional laboratories will be re-equipped, and the routine analytical work will be standarized and rationalized. The analytical results will be calibrated in relation to the results of the field experiments.

In order to facilitate comordination of countrywide results, the soil types of all experimental and demonstration sites will be identified, and yield results grouped in accordance with these findings, or, with existing soil maps if they are available.

The correlations between soil types, soll analysis, and fertilizer responses will be 
establiahed in comperation between the project and the appropriate Research Institute involved in soils work.

Benefit/cost studies should be undertaken on fertilizer use and improved soil management, and cultivation practices based on data of previous experiments and those," collected by the project.

Fertilizer recommendations for farmers will be worked out, based on field experiments, greenhouse and laboratory work. The recommendations for the various crops will be reviewed yearly in the light of experimental results.

Co-operation with the extension service with regard to fertilizer demonstrations, will be intensified by joint meetings, lectures, seminars and publications. This will enable the extension to : a) show the farmers the physical and economic effects of fertilizers; b) help the farmers to buy fertilizers, and c) to teach them how to apply the fertilizers correctly.

\subsection{Applied Research Programmes on Specific Soil Fertility and Fertilizer \\ Problems}

Research and experimental work on more specific soil fertility problems will be carried out in co-operation with the Soil Research Institute or other appropriate Research Stations.

Exact field experiments will be carried out to determine:

a) residual and cumulative effects of $N, P$ and $K$ and their interaction on the quantity and quality of the produce;

b) comparison of the relative efficiency of different nitrogen and phosphorus carriers and of compound fertilizers;

c) experiments on time and methods of fertilizer application;

d) effects of organic manures in combination with fertilizers, and

e) study on low phosphate availability.

Greenhouse studies will be made to determine:

a) effects of secondary and micronutrient elements, and

b) calibration of soil tests.

A programme of research and demonstration on soil management and related cultural practices will be established covering the following topics:
a) high yielding varieties;
b) row planting versus broadcasting methods;
c) increased plant population;
d) weed control, and
e) plant protection. 
Besed on the technical and economic information obtajned by the field experimental work, advice should be given to the Government on fertilizer, price policy, subsidy and taxation.

The project should provide the Govermment with realiatic technical and economic datai for the formulation of its long term fertilizer policy.

A study should be prepared on the quantities and types of fertilizers, and the economic aspects as a basis for the plannine of local fertilizer production. Depending on the conclusions reached, a technological feasibility study on fertilizer manufacturing should be carried out.

Fertilizer legislation should cover the following:

a) licensing requirements regarding manufacture, import and sale of fertilizers;

b) declaration to be made as to plant nutrient content;

c) analytical methods to be used for determining the various nutrients;

d) official inspection and control measures, and

e) penalties for noncompliance with the provisions of legislation.

\subsection{Training in Soil Fertility and Fertilizer Work}

The training of local personnel should consist of on the job training; training courses in the country, and fellowship and study trips outside the country. It should also cover all activities of a project.

Counterpart staff at all levels should take part in the training. Extension service staff should be trained in fertilizer use practices, and know the suitable means for publicizing these practices and supervising their application. Senior officers, responsible for the planning of long term fertilizer use development in the country, should have the possibility of familiarizing themselves with the most efficient and up to date methods in this field.

\subsection{Fertilizer Use}

The work done so far in the project on soil fertility and fertilizer use in Afghanistan, is not sufficient to make specific recommendations for fertilizer use for all regions and all crops of the country. However, the results obtained serve as very useful indicators of the possible magnitude of responses to some crops in some regions of Afghanistan. These results may also form the basis for planning a long-term programme of soil fertility and fertilizer use for the entire country.

Therefore, it is strongly recommended that the work of the existing project be expanded and extended to other regions of Afghanistan. It is also suggested that a long term programe of establishing a Soll Fertility Unit, should be adopted to assist the Government in planning the use of chemical fertilizers and in developing sound economic and agricultural policy.

\section{7 .1 Objectives}

It is suggested that the Soil Fertility Unit be set up with the following 
objectives:

- To study responses to nitrogen, phosphorus and potassium appized alone, and in comoinstion with al the important cereal, industrial, horticultural and vegetebie crope. of the country under irrigated and un-iriketed conditions of farming, and to work out the $:$ feritizor requirements of eil the crope.

- To carry out a thorough soil fertility survey of important soil types of the coutry, and work out soil test-crop response correlation as an ajd to develop practical recomendations to the farmers.

- Mo collect economio appraisai of fertilizer use and to demonstrate to the fermere economics of fertilizer lise in increasing crop production.

- To assist the Goverment in making policies regarding the kinds and anounts of fertilizers to be imported, and explore the possibilities of manufacturing fertiizers.

- To assist tre Govemment in carying out the programme for fertilizer storase, inatribution, marketing and crecit, and in formulating fentilizer control.

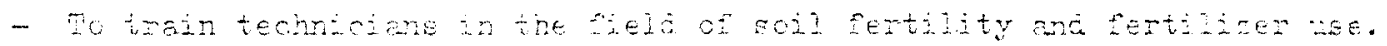

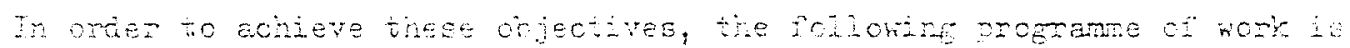

angestas:

e) to plar and conduc a roring progneme to coliect information from the comolex fertilizer experiments to be carriod out for three years (1970 to 1973 ), at selected research farms in the regions of:

$$
\begin{aligned}
& \text { - Käbul } \\
& \text { - Kandahar } \\
& \text { - Herat } \\
& \text { - Balkh } \\
& \text { - Kunduz } \\
& \text { - Nengarhar }
\end{aligned}
$$

These experiments would provide solutions to the following problems:

(i) the kinds of nitrogenous, phosphorus and potassic fertilizers which would be best for the country;

(ii) the optimum rate of their application, and the best method and time to apply them to various crops, and

(iii) the residual values of phosphatic fertilizers.

In order to know how much fertilizer to apply under irrigated and unirrigated conditions, the interaction of moisture levels and fertilizers will have to be studied. Also the interaction of variety and fertilizers will have to be studied to find out the fertilizer needs of high yielding varieties of various crops.

b) To plan and conduct a working programme to collect information from the simple 12 plot $(3 \times 3$ factorial for nitrogen and phosphorus +3 checkplots for $K$ ), experiments on farmers' fields (irrigated and dry land), in order to work out the responses under the field conditions in the following regions:

Region No. Is Comprising Kabul, Ghazani, Maidan, Logar, Parwan, Kapisa, Bamyan, Ruzgan and Paktia Provinces. 
Region No. II: Comprising Kandahar, Helmand, Zabul, Farah and Chakansur Provinces.

Region No. IIT: Comprising Herat, Badhis and Ghor Provinces

Region No. IV: Comprising Balkh, Faryab, Samanghan and Jozjan Provinces

Region No. V: Comprising Kunduz, Baghlan, Badakshan and Takhar Provinces.

Region No. VI: Comprising Nangarhar, Lagman and Kunar Provinces.

The following progranme is suggested for each region:

\begin{tabular}{|c|c|c|c|}
\hline Region No. & Crop & $\begin{array}{l}\text { Number of trials } \\
\text { per year }\end{array}$ & Year \\
\hline I & $\begin{array}{l}\text { Wheat } \\
\text { Corn }\end{array}$ & $\begin{array}{l}500 \\
200 \\
\end{array}$ & $\begin{array}{r}1970-1971 \\
1970-1972 \\
\end{array}$ \\
\hline II & $\begin{array}{l}\text { Wheat } \\
\text { Corn } \\
\text { Rice }\end{array}$ & $\begin{array}{l}300 \\
200 \\
100 \\
\end{array}$ & $\begin{array}{l}1970-1973 \\
1970-1973 \\
1970-1973 \\
\end{array}$ \\
\hline III & $\begin{array}{l}\text { Wheat } \\
\text { Corn } \\
\text { Rice }\end{array}$ & $\begin{array}{l}300 \\
100 \\
100 \\
\end{array}$ & $\begin{array}{l}1970-1973 \\
1970-1973 \\
1970-1973 \\
\end{array}$ \\
\hline IV & $\begin{array}{l}\text { Wheat } \\
\text { Cotton } \\
\text { Sugarbeet }\end{array}$ & $\begin{array}{r}300 \\
200 \\
50 \\
\end{array}$ & $\begin{array}{r}1970-1973 \\
1970-1973 \\
1970-1973 \\
\end{array}$ \\
\hline$v$ & $\begin{array}{l}\text { Wheat } \\
\text { Rice } \\
\text { Cotton } \\
\text { Sugarbeet }\end{array}$ & $\begin{array}{l}300 \\
200 \\
200 \\
200 \\
\end{array}$ & $\begin{array}{l}1970-1973 \\
1970-1973 \\
1970-1973 \\
1970-1973\end{array}$ \\
\hline VI & $\begin{array}{l}\text { Wheat } \\
\text { Rice } \\
\text { Sugarcane }\end{array}$ & $\begin{array}{r}300 \\
300 \\
50 \\
\end{array}$ & $\begin{array}{c}1970-1972 \\
1970-1973 \\
1970-1973 \\
\end{array}$ \\
\hline
\end{tabular}

c) To set up a modern and efficient soil testing service laboratory.

The existing laboratory of the Ministry should be strengthened and reoriented to work as an efficient soil testing service for the Afghan farmer, where he can get the soils of his farm tested, and can obtain fertilizer recomendations on the basis of
soil tests. The responsibility of the laboratory would be to: (1) oolleot large numbers of representative soil samples for
analysis; 
(ii) onopt nopire and accurate proodures for doil anaigris;

(iij) interpret goil analyeis data in regpect to availability of nutrients to the crope;

(iv) corry out soil test-orop zemporse cormelation rork;

(v) recommend fertilizer use on the basis of ail teste; and

(vi) prepare soil fertility mape of different regions of the country and report fertility status changes fron time to tine.

(1) To set up a sub-uit'to carry out the etuag of the problene of storage, distribution, marketing and credit facilities for the fertilizers, and auggegt ways and means of streaminirig theat procedures.

e) Organize and concuct training courses for Arehan technicians in planning, conducting and data collectirig fror: .

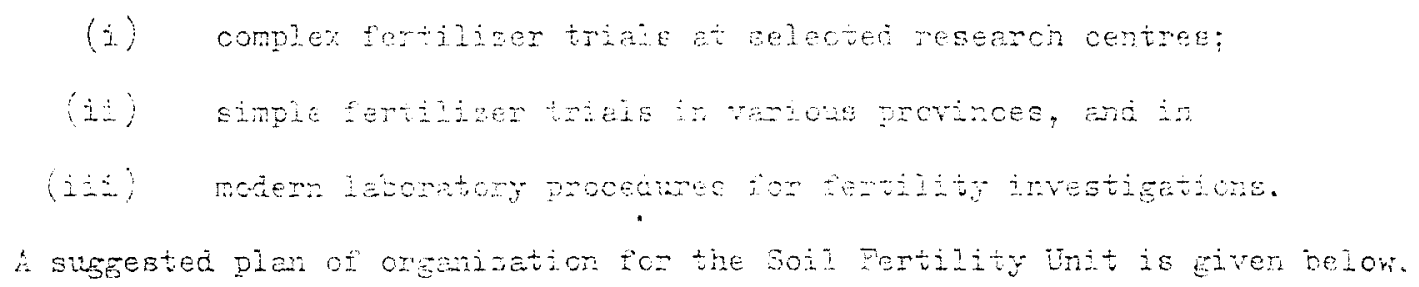

SOII PRTTHITY UNTT

\begin{tabular}{|l|l|l|l|l|}
\hline $\begin{array}{l}\text { Section for } \\
\text { complex trials } \\
\text { at } 6 \text { research } \\
\text { stations }\end{array}$ & $\begin{array}{l}\text { Section for } \\
\text { simple trials } \\
\text { on farmers'fields } \\
\text { in 6 regions }\end{array}$ & $\begin{array}{l}\text { Soil testing } \\
\text { service } \\
\text { laboratory }\end{array}$ & $\begin{array}{l}\text { section for } \\
\text { study of fertilizer } \\
\text { storage, distribution } \\
\text { marketing and credit } \\
\text { etc. }\end{array}$ \\
\hline
\end{tabular}

The field work at the Research Station should be done by the special staff assigned for this work in collaboration with the Director of the Research Station.

The Directors-general should be responsible for carrying out the field work in the provinces with the help of the special staff to be stationed in these provinces.

The Director of the Soil Testing Laboratory should be responsible to the Chief of the Soil Fertility Unit.

\subsubsection{Advice to farmers} to the farmers:

On the basis of the work done by the expert, the following advice may be given

a) The improved and high yielding varieties should be used since they yield much higher than the local varieties.

b) Although the application of nitrogen and phosphorus alone gives significant increase over the oontrol ylelds in most cases, the farmers should never use nitrogen 
or phosphorus alone since it is not economical.

c) Highest yields are always obtained when $\mathrm{N}$ and $\mathrm{P}_{2} \mathrm{O}_{5}$ have been used in the ratio of 1:1; other ratios $(2: 1$ or $1: 2)$ did not give economic yields. Therefore, the, ratio $1: 1$ is recommended. 
APFENDIX I

PEFERENCES

\begin{tabular}{|c|c|}
\hline 亿́ & $\begin{array}{c}\text { Alierson, G. } \\
1967\end{array}$ \\
\hline 2. & $\begin{array}{c}\text { Carter, D.P. } \\
1966\end{array}$ \\
\hline s. & $\begin{array}{r}\text { Carter, C.P. } \\
1967\end{array}$ \\
\hline 4 & $\begin{array}{c}\text { Frutchey, G.id. } \\
1962\end{array}$ \\
\hline$\therefore$, & Ho, Some \\
\hline & $\begin{array}{r}340 / 35 \\
1965\end{array}$ \\
\hline 7. & $\begin{array}{c}\text { Hauser, G. } \\
1953\end{array}$ \\
\hline 8. & $\begin{array}{l}\text { Hauser, } \mathbf{G} . \\
1952-1953\end{array}$ \\
\hline 9. & $\begin{array}{r}\text { Hauser, } \\
1953^{\circ}\end{array}$ \\
\hline 10. & $\underset{1965}{\text { Kumler, }}$ \\
\hline
\end{tabular}

11. Mortensen, E. 1967

12. Mortensen, E. $1966-1968$

13. Natiaqui, H.A.
1968
The Place of Cotton and Sugarbet in Afghat Economy, Kaiul, Britiah Embassy, 69 p. mineo.

Hervest Results of Extension. Farmer Demonstration in Helmand Valley Authority. USAID Agriculture, Bogt, Af phanietar.

Felmand-Arghandab Velley Authority. Extendion Spongored Com Demonstration Report. USAD, Boat, Afgharietan. 8 p. ditto and mimeo.

Semi-Amual Report - USOM, hyoning, Kroul, Afghanistan. $17 \mathrm{j}$.

Production Year Eoom, VoI. Ẑ́.

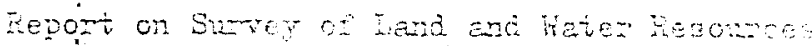
ffghenisten, Vol. Ir, $9 /$ AKr. Rome.

Report of Laboratory Hork. Ninistry of Agriculture, Kabul, Afghanistan. $12 p$.

Report about the General Sugerbeet Cultivation at Baghlan, and results of Beet Experiments in Field. 9 p. ditto.

Sugarbeet Cultivation in Afghanistan and its Improvement. Empire Joumal of Experiment and Agriculture.

Fertilizer Trials using Six Samples taken from Plots where Levelling Occurred. Faculty of Agriculture Report. Kabul University, Afghanistan. 8 p. mimeo.

Monthly Report for June 1967. USAD, Agriculture Jalalabad, Afghanistan.

Review of Rice Research Conducted at Shisam Bagh Experiment Station, Jalalabad, MAI and USATD, Kabul, Afghanistan. Mimeo.

Effect of Fertilizer and Cultaral Methods upon Local and Introduced Variet1es. Tech. Bull. No. 4. Kabul University, Faoulty of Agriculture, Afghantatan. $16 \mathrm{p}$. 
14. Nelson, E.M.
1962
15. Nielson, G.A.
1964

16. Royal Afghan Govermment Ministry of Planning 1967

17. Salem, M.Z.

18. Semin, A.Q. 1965-1966

19. Samin, A.Q. 1967

20. Samin, A.Q. 1968

21. Shuman, F.H. 1959

22. Shuman, F.H. 1959-1960

23. Shuman, F.H. 1960

24. US Agriculture Review Team 1967

25. USAID - Ministry of Agriculture and Irrigation 1969

26. Vaughn, J.R. 1961
Wheat Fertilizer Mrial Results, Kabul Area. USADl Agriculture, Kabul, Afghanigtan, 2 p. ditto.

Progress Report - Fertility Studies on Corn. Faculty of Agricuiture, Kabul Univeraity, Afghanistan. 4 p. mimeo.

The Third Five Year Economic and Social Plan of Afghanistan.

Soil Geography and Factors of Soil Formation in Afghanistan. Social Science Vol. 197.

Corn Yields in Logar and Kabul Provinces. Faculty of Africulture, Kabul University, Afghanistan.

Fertilizer Triels on wheat in Afghanistan. Tech. Bull. No. 1. Kabul University, Afghanistan.

Fertilizer Trials on Corn. Teoh. Bull. No. 10, Kabul University, Faculty of Agriculture, Afghanistan.

Report of the Responses to Wheat to Fertilizers, Village Trials. ICA Report. Kabul, Afghanistan.

Extension with Wheat. ICA Report. Kabul, Afghanistan.

Com needs Improvement, a Guide for Future. USOM, Afghanistan.

Agrioultural Development in Afghanistan with Speoial Emphasis on theat Problem.

Fourth Annual Wheat Seminar Proceedings Kabul, Afghanistan.

Semi-Annual Report. University of Wyoming Contract. Kabul, Afghanistan. 
APFENDIX II

ANALYSIS OF VARIAHCE

Lagminan

(a) Wheat - 1968-69

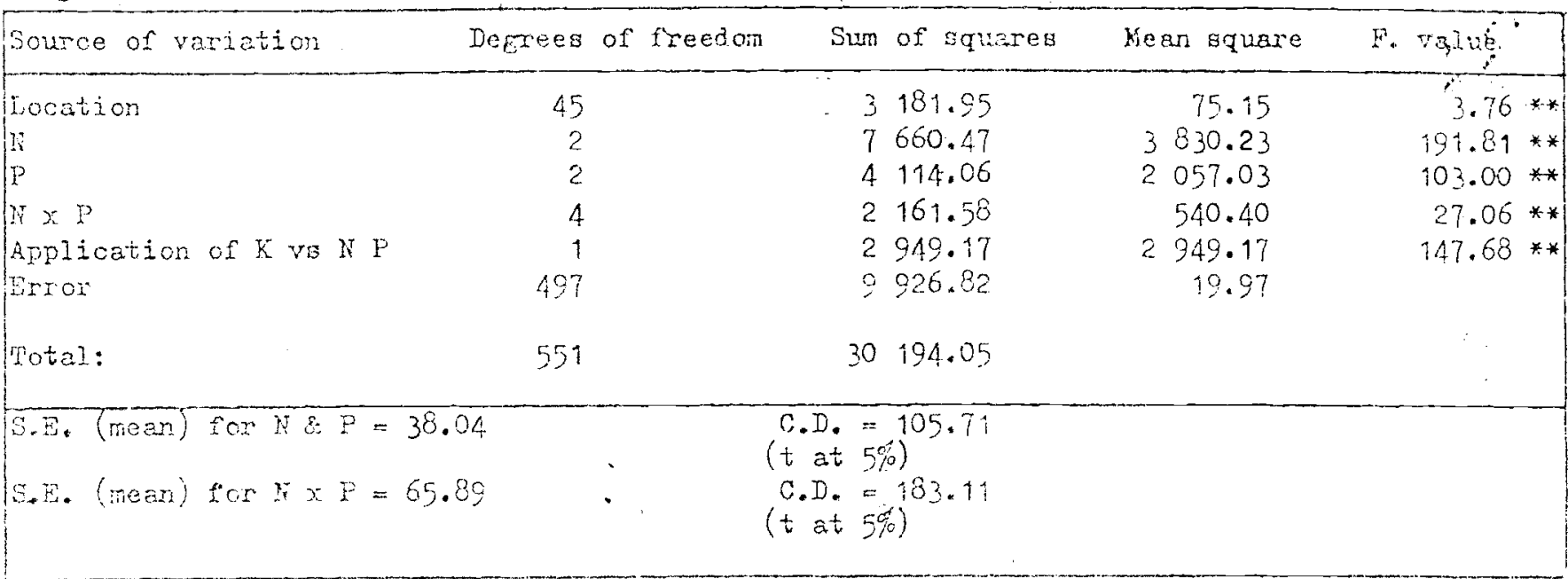

\section{Mangarisan}

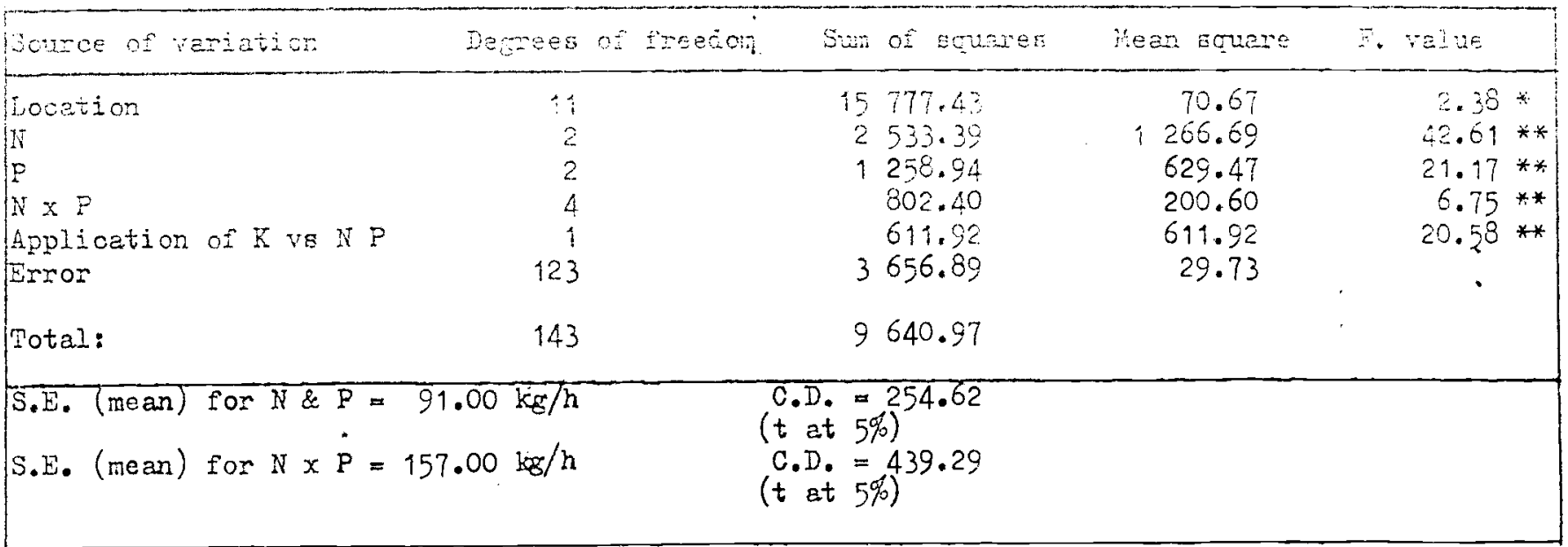

Kabul

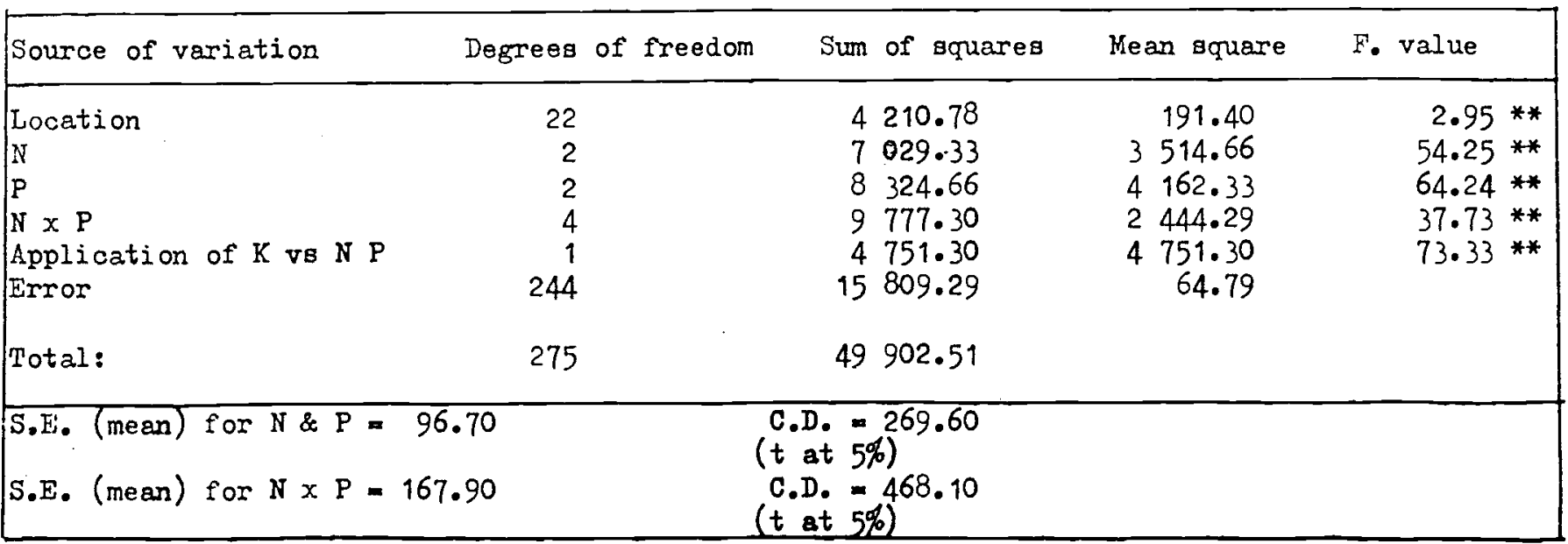


Kapisa end Parwan

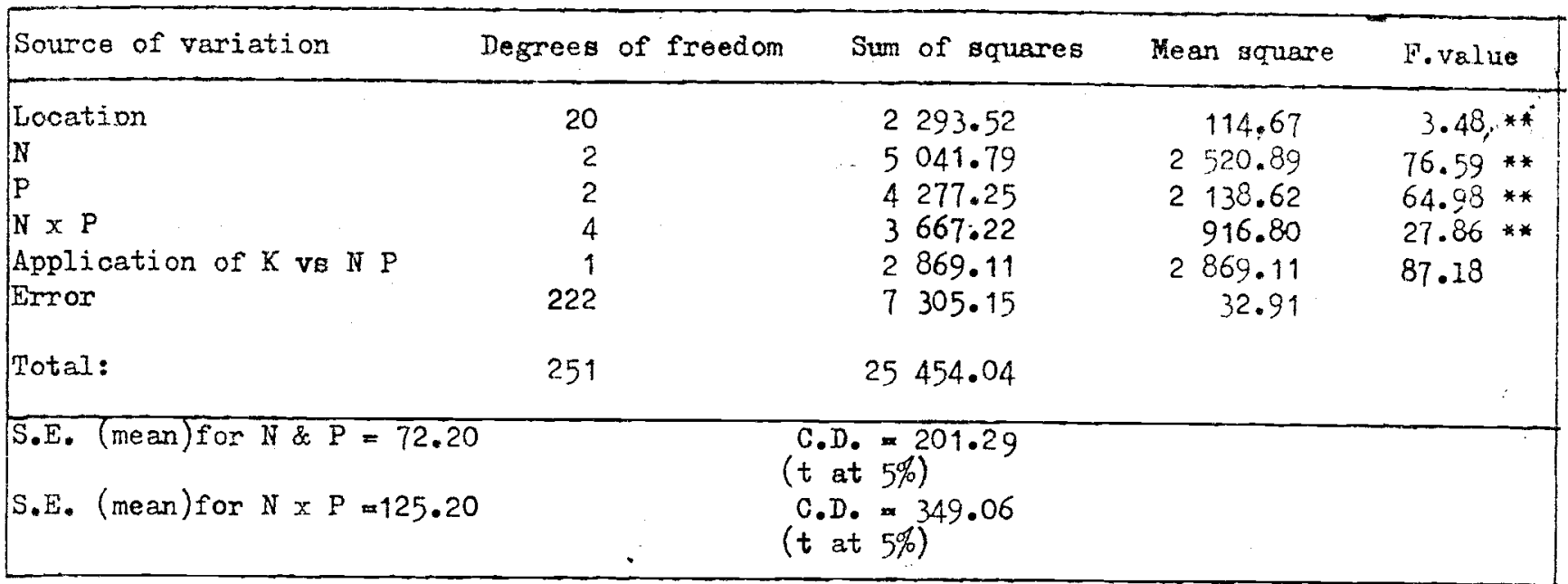

Logar

\begin{tabular}{|c|c|c|c|c|}
\hline Source of variation & Degrees of freedom & Sum of squares & Hean square & F. value \\
\hline $\begin{array}{l}\text { Location } \\
\mathrm{N} \\
\mathrm{P} \\
\mathrm{N} \times \mathrm{P} \\
\text { Application of } \mathrm{K} \text { ve N P } \\
\text { Error }\end{array}$ & $\begin{array}{r}12 \\
2 \\
2 \\
4 \\
1 \\
134\end{array}$ & $\begin{array}{ll} & 930.64 \\
3 & 435.38 \\
3 & 241.42 \\
4 & 510.01 \\
1 & 852.93 \\
6 & 530.16\end{array}$ & $\begin{array}{rr} & 77.55 \\
1 & 717.69 \\
1 & 625.71 \\
1 & 127.50 \\
1 & 852.93 \\
& 48.73\end{array}$ & $\begin{array}{l}1.59 \text { Sig } \\
35.25 * * \\
33.36 * * \\
23.14 * * \\
38.02 * *\end{array}$ \\
\hline Total: & 155 & 20500.54 & & \\
\hline $\begin{array}{l}\text { S.E. (mean) for } N \& P= \\
\text { S.E. (mean) for } N \times P .=\end{array}$ & & $\begin{array}{l}\text { D. }=311.42 \\
\text { st } 5 \%) \\
\text { D. }=541.41 \\
\text { at } 5 \%)\end{array}$ & & \\
\hline
\end{tabular}

\section{Maidan}

\begin{tabular}{|c|c|c|c|c|}
\hline Source of variation & Degrees of freedom & Sum of squares & Mean square & F. value \\
\hline $\begin{array}{l}\text { Location } \\
N \\
P \\
N \\
N \text { P } \\
\text { Application of } \mathrm{K} \text { va N P } \\
\text { Error }\end{array}$ & $\begin{array}{r}9 \\
2 \\
2 \\
4 \\
1 \\
101\end{array}$ & $\begin{array}{ll} & 361.04 \\
3 & 914.93 \\
4 & 128.60 \\
3 & 503.40 \\
1 & 200.15 \\
6 & 036.68\end{array}$ & $\begin{array}{rr} & 40.11 \\
1 & 957.46 \\
2 & 064 \cdot 30 \\
& 875.85 \\
1 & 200.15 \\
& 59.77\end{array}$ & $\begin{array}{l}\text { Not Sig. } \\
32.75^{* *} \\
34.54 * * \\
14.65^{* *} \\
20.08 * *\end{array}$ \\
\hline T'otal: & 119 & 19144.80 & & \\
\hline $\begin{array}{l}\text { S.E. (mean) for } N \& P= \\
\text { S.E. (mean) for } N \times P=\end{array}$ & \multicolumn{2}{|c|}{$\begin{array}{l}\text { C.D. }=395.78 \\
(t \text { at } 5 \%) \\
\text { C.D. }=687.23 \\
(t \text { at } 5 \%)\end{array}$} & & \\
\hline
\end{tabular}


Kabul Dry-Länd

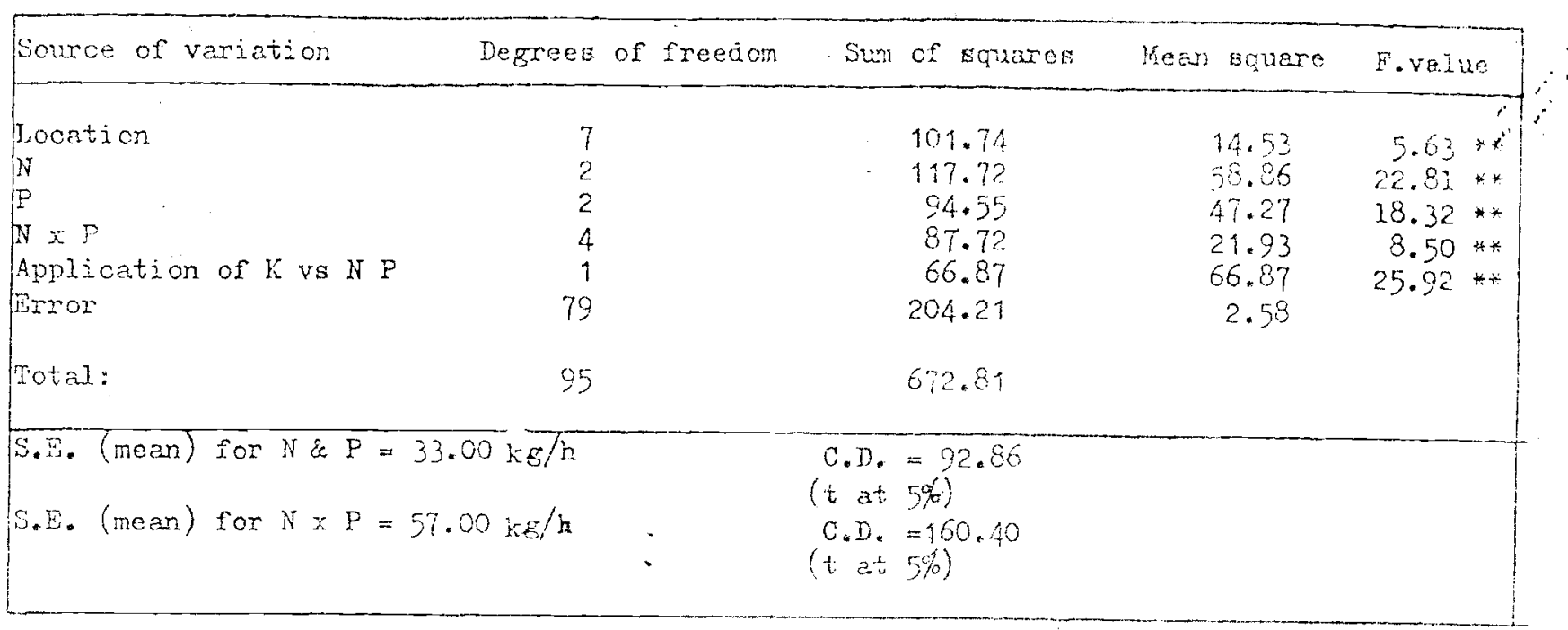


APPENDIX II

ANALYSIS OF VARIAHCE

(b) Wheat - 1969-70

Lagman

\begin{tabular}{|c|c|c|c|c|c|}
\hline Source of variation & Degrees of freedom & Sum of squares & Mean square & Obs. & $\begin{array}{l}\text { value } \\
\text { Actual }\end{array}$ \\
\hline $\begin{array}{l}\text { Location } \\
\text { N } \\
P \\
N \text { X } \\
\text { Application of } K \text { va NP } \\
\text { Error }\end{array}$ & $\begin{array}{r}44 \\
2 \\
2 \\
4 \\
1 \\
486\end{array}$ & $\begin{array}{rl}5 & 775.18 \\
9 & 173.00 \\
1 & 678.90 \\
7 & 828.90 \\
5 & 045.00 \\
60 & 248.71\end{array}$ & $\begin{array}{ll} & 131.12 \\
4 & 585.00 \\
& 839.4 \\
1 & 957.0 \\
5 & 045.0 \\
124 \cdot 0\end{array}$ & $\begin{array}{r}1.0 \\
36.98 \\
6.76 \\
15.78 \\
40.68\end{array}$ & $\begin{array}{l}1.60 \\
4.60 \\
4.60 \\
3.35 \\
6.68\end{array}$ \\
\hline Total: & 539 & 89785.69 & & & \\
\hline
\end{tabular}

Nangarhar

\begin{tabular}{|c|c|c|c|c|c|}
\hline \multirow[t]{2}{*}{ Source of variation } & \multirow[t]{2}{*}{ Degrees of freedom } & \multirow[t]{2}{*}{ Sun of squares } & \multirow{2}{*}{ Mean square } & \multicolumn{2}{|c|}{ F. value } \\
\hline & & & & Obs. & Actual \\
\hline Location & 48 & 4645.02 & 96.7 & 1.09 & 1.55 \\
\hline $\mathrm{N}$ & 2 & 8578.90 & 4289.0 & 48.67 & 4.64 \\
\hline$P$ & 2 & 6888.20 & 3444.0 & 39.14 & 4.64 \\
\hline $\mathrm{N} \times \mathrm{P}$ & 4 & 10050.50 & 2513.0 & 28.56 & 3.35 \\
\hline Application of $\mathrm{K}$ vs.NP & 1 & 3841.00 & 3841.0 & 43.64 & 6.68 \\
\hline Error & 530 & 46824.55 & 88.34 & - & - \\
\hline Total: & 587 & 80828.17 & - & - & - \\
\hline $\begin{array}{ll}\text { S.E. }(\text { mean }) & =59.00 \\
\text { C.D. } & =152.22\end{array}$ & & & & & \\
\hline
\end{tabular}

KabuI

\begin{tabular}{|c|c|c|c|c|c|}
\hline Source of variation & Degrees of freedom & Sum of squares & Mean Square & $\begin{array}{r}F . \\
\text { Obs. }\end{array}$ & $\begin{array}{l}\text { value } \\
\text { Actual I }\end{array}$ \\
\hline $\begin{array}{l}\text { Location } \\
N \\
\mathrm{P} \\
\mathrm{N} \text { x P } \\
\text { Application of K vo N P } \\
\text { Error }\end{array}$ & $\begin{array}{r}28 \\
2 \\
2 \\
4 \\
1 \\
310\end{array}$ & $\begin{array}{rr}2 & 930.52 \\
9 & 285.10 \\
4 & 868.70 \\
3 & 811.60 \\
1 & 344.80 \\
28 & 894.24\end{array}$ & $\begin{array}{rr} & 104.66 \\
4 & 642.55 \\
2 & 434.35 \\
952.90 \\
1 & 344.80 \\
& 93.20\end{array}$ & $\begin{array}{r}1.12 \\
49.81 \\
26.12 \\
10.22 \\
14.42 \\
-\end{array}$ & $\begin{array}{l}1.72 \\
4.68 \\
4.68 \\
3.36 \\
6.70 \\
-\end{array}$ \\
\hline Total & 347 & $51 \quad 134.96$ & - & - & - \\
\hline
\end{tabular}


Kapiea

\begin{tabular}{|c|c|c|c|c|c|}
\hline Source of veriation & Degrees of freedom & Sum of aquares & Mean square & $\begin{array}{c}F \cdot \\
\text { Obas. }\end{array}$ & $\begin{array}{l}\text { Value } \\
\text { kctual ' } 19\end{array}$ \\
\hline Location & 41 & 3649.11 & 89.00 & 1.24 & $\therefore 6$ \\
\hline$y^{i}$ & 2 & $83.36 \cdot 30$ & 4168.15 & 67.55 & 4.66 \\
\hline P & 2 & 5212.90 & 2606.45 & 42.24 & 4.66 \\
\hline IN $x \mathrm{P}$ & 4 & 3171.10 & 792.77 & 12.84 & 3.36 \\
\hline Application of $K$ vs N P & 1 & 9540.50 & 9540.50 & 154.60 & 6.66 \\
\hline Wrror & 453 & 27950.99 & 69.70 & - & - \\
\hline Total: & 503 & 57860.90 & $\hat{=}$ & - & - \\
\hline $\begin{aligned}\text { S.E. (mesn }) & =65.38 \\
\text { C.D. } & =168.68\end{aligned}$ & & & & & \\
\hline
\end{tabular}

\section{Parwan}

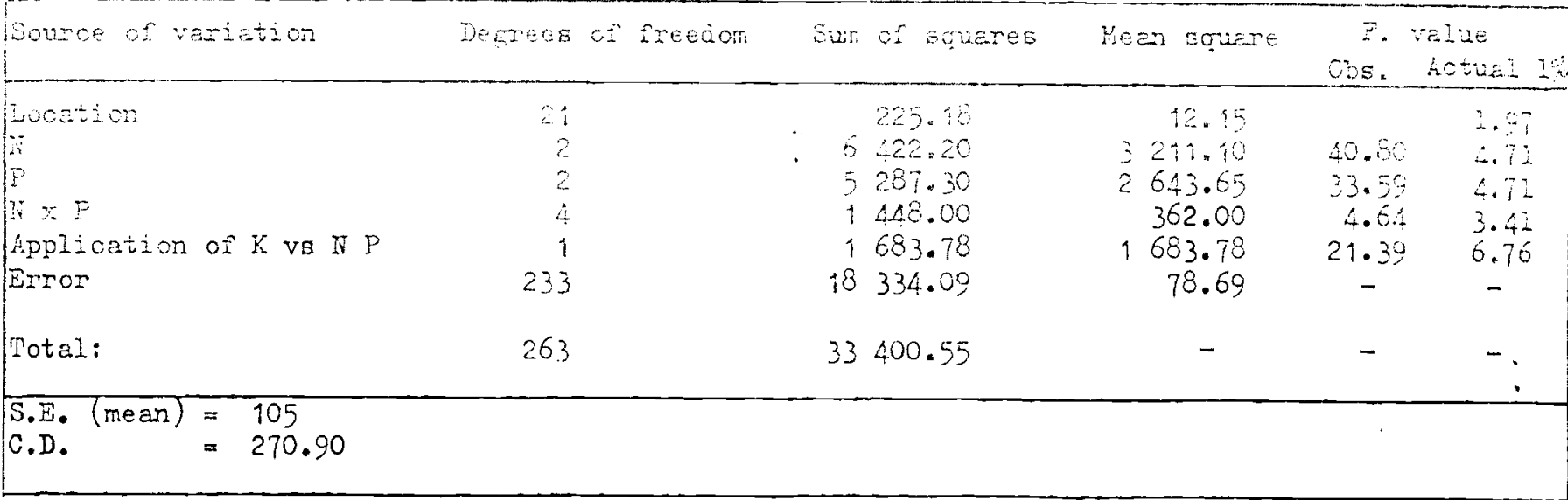

Logar

\begin{tabular}{|c|c|c|c|c|c|}
\hline Source of variation & Degrees of freedon & Sum of squares & Mean square & $\begin{array}{l}\text { F. V } \\
\text { Obs. }\end{array}$ & $\begin{array}{l}\text { value } \\
\text { Actual } 1 \%\end{array}$ \\
\hline $\begin{array}{l}\text { Location } \\
N \\
P \\
N \text { x } P \\
\text { Application of } K \text { vB N P } \\
\text { Error }\end{array}$ & $\begin{array}{r}34 \\
2 \\
2 \\
4 \\
1 \\
377\end{array}$ & $\begin{array}{rr} & 218.84 \\
7 & 371.13 \\
2 & 818.24 \\
2 & 528.58 \\
& 251.92 \\
24 & 097.29\end{array}$ & $\begin{array}{rr} & 6.43 \\
3 & 685.50 \\
1 & 409.12 \\
632.14 \\
251.92 \\
63.91\end{array}$ & $\begin{array}{c}57.66 \\
22.04 \\
9.89 \\
3.94 \\
-\end{array}$ & $\begin{array}{l}1.64 \\
4.66 \\
4.66 \\
3.36 \\
3.86 \\
-\end{array}$ \\
\hline Total: & 420 & 37286.00 & - & - & - \\
\hline $\begin{array}{ll}\text { S.E. }(\text { mean }) & =67.16 \\
\text { C.D. } & =173.27\end{array}$ & & & & & \\
\hline
\end{tabular}


Maidan

\begin{tabular}{|c|c|c|c|c|c|}
\hline Source of variation & Degrees of freedom & Sun of squares & Mean Bouare & $\begin{array}{r}\text { F. } \\
\text { Obe. } \\
\end{array}$ & $\begin{array}{l}\text { alue } \\
\text { Aotual } 1\end{array}$ \\
\hline $\begin{array}{l}\text { Location } \\
N \\
P \\
N \times P \\
\text { Application of } K \text { vs } N P \\
\text { Error } \\
\text { Total: }\end{array}$ & $\begin{array}{r}15 \\
2 \\
2 \\
4 \\
1 \\
168 \\
192\end{array}$ & $\begin{array}{rr}1 & 462.29 \\
4 & 713.77 \\
1 & 415.00 \\
1 & 575.22 \\
1 & 207.32 \\
10 & 843.08 \\
21 & 016.68\end{array}$ & $\begin{array}{r}84.15 \\
2 \quad 356.88 \\
707.50 \\
398.80 \\
1207.32 \\
64.54\end{array}$ & $\begin{array}{r}1.30 \\
36.51 \\
10.96 \\
6.10 \\
18.70\end{array}$ & $\begin{array}{l}2.10 \\
4.72 \\
4.72 \\
3.42 \\
6.80\end{array}$ \\
\hline
\end{tabular}

S.E. (mean) $=114$

C.D. $\quad=294.12$

Baghlen

\begin{tabular}{|c|c|c|c|c|c|c|}
\hline Source of variation & Degreeg of & freadon & Sum of squares & Mean aquare & $\begin{array}{l}P . V \\
\mathrm{Ob} \text {. }\end{array}$ & $\begin{array}{l}\text { Alue } \\
\text { Actual } 2 \%\end{array}$ \\
\hline $\begin{array}{l}\text { Location } \\
N \\
P \\
N \text { x } \\
\text { Application K v8 N P } \\
\text { Error } \\
\text { Total: }\end{array}$ & $\begin{array}{r}30 \\
2 \\
2 \\
4 \\
1 \\
333 \\
372\end{array}$ & - & $\begin{array}{ll}2 & 370.54 \\
9 & 443.30 \\
1 & 240.58 \\
5 & 004.20 \\
& 371.05 \\
45 & 502.83 \\
63 & 932.50\end{array}$ & $\begin{array}{r}79.00 \\
4721.65 \\
620.29 \\
1251.05 \\
371.05 \\
136.64\end{array}$ & $\begin{array}{r}0.58 \\
34.55 \\
4.54 \\
9.15 \\
2.72\end{array}$ & $\begin{array}{l}1.75 \\
4.68 \\
3.02 \\
3.38 \\
3.88\end{array}$ \\
\hline
\end{tabular}

$\begin{array}{ll}\text { S.E. (mean) } & =92.00 \\ \text { C.D. } & =237.36\end{array}$ 

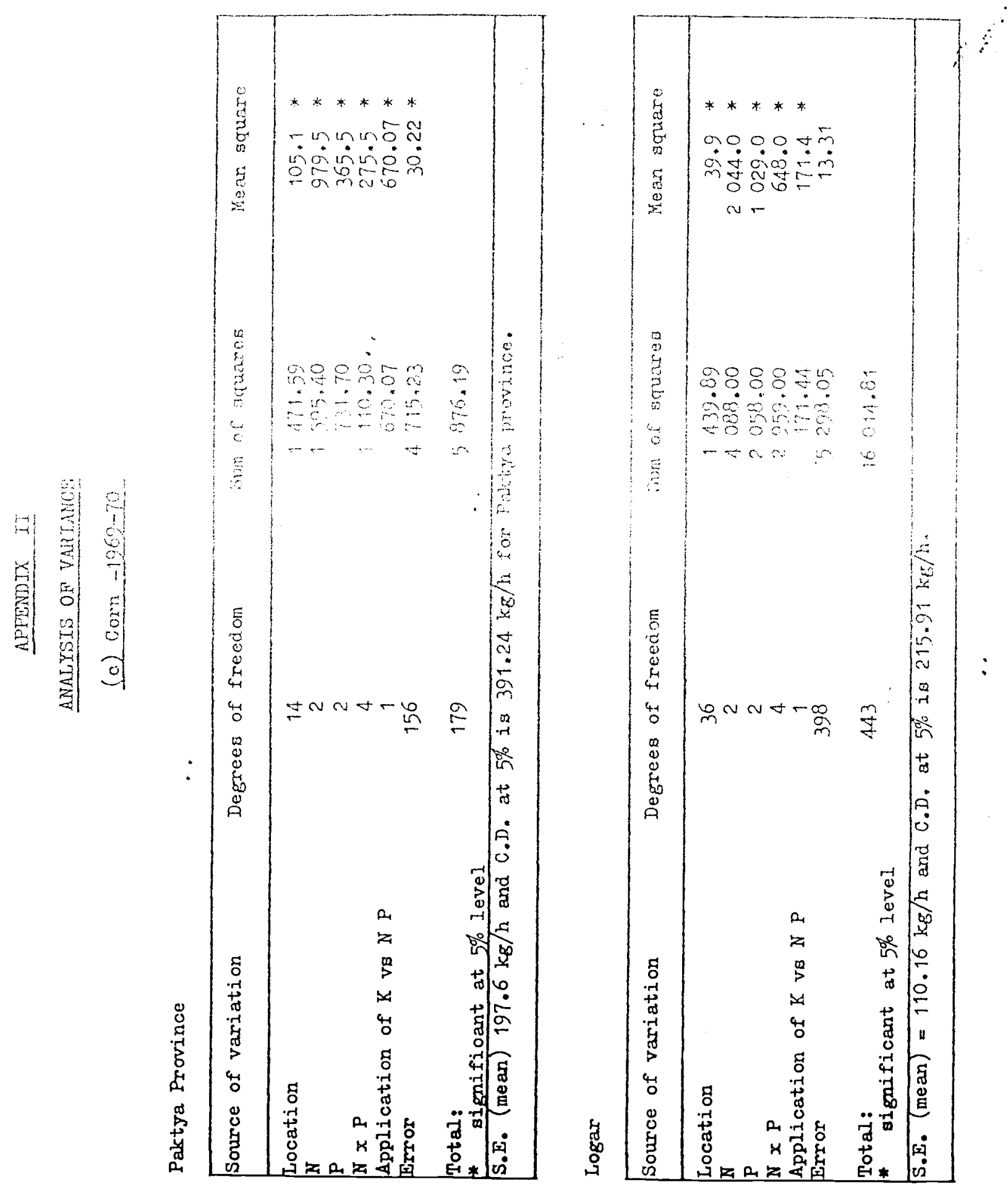


\begin{tabular}{|c|c|c|c|c|c|c|c|c|c|}
\hline \multirow{7}{*}{ 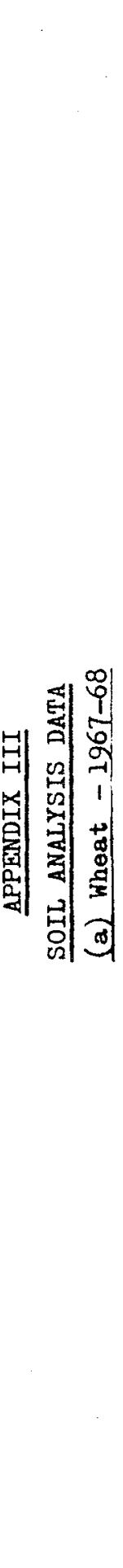 } & 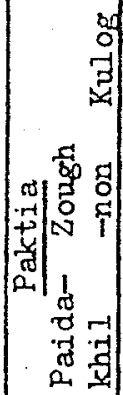 & 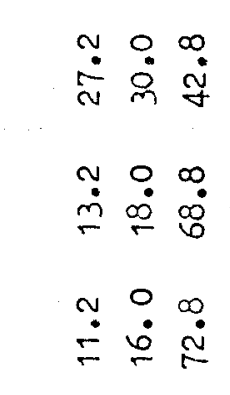 & 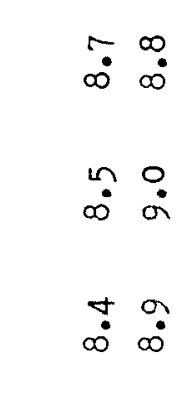 & $\begin{array}{l}\dot{0} \\
\dot{0} \\
m \\
\dot{0} \\
\dot{0} \\
\dot{0}\end{array}$ & $\begin{array}{l}\stackrel{n}{0} \\
0 \\
\dot{0} \\
+ \\
\dot{0}\end{array}$ & 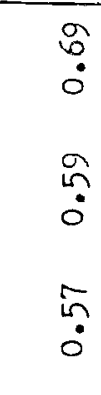 & $\begin{array}{l}\stackrel{\circ}{\circ} \\
\stackrel{+}{\circ}\end{array}$ & $\begin{array}{l}\AA^{\prime} \\
\stackrel{N}{\mathbb{N}}\end{array}$ & 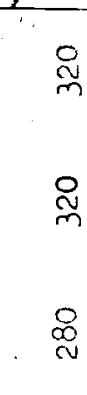 \\
\hline & 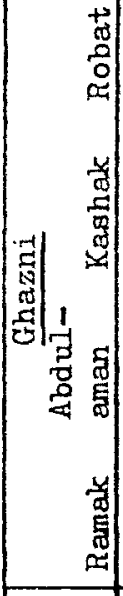 & 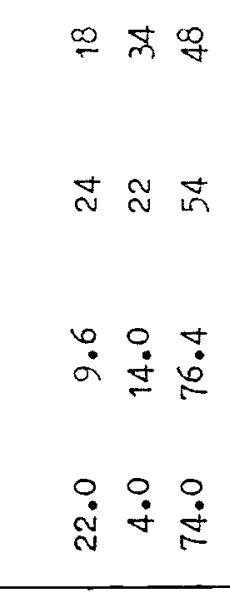 & 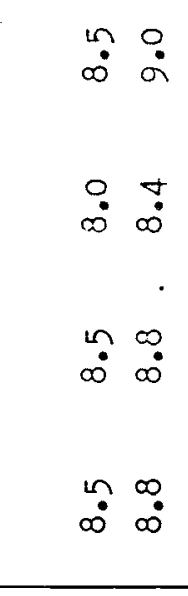 & $\begin{array}{l}\dot{m} \\
\dot{0} \\
\dot{0} \\
\dot{0} \\
\hat{0} \\
\dot{0} \\
\hat{0} \\
\dot{0}\end{array}$ & $\begin{array}{l}\stackrel{\dot{0}}{-} \\
\dot{\square} \\
\dot{\sim} \\
\dot{0} \\
\infty\end{array}$ & $\begin{array}{l}m \\
\ddot{0} \\
\stackrel{0}{\dot{0}} \\
\dot{m} \\
\dot{0} \\
\ddot{m} \\
\dot{0}\end{array}$ & $\begin{array}{l}\stackrel{\infty}{N} \\
\stackrel{\infty}{\infty}\end{array}$ & $\begin{array}{l}\stackrel{\oplus}{ } \\
\cong\end{array}$ & $\begin{array}{l}\stackrel{8}{m} \\
\stackrel{\circ}{\sim} \\
\stackrel{\circ}{\circ} \\
\frac{\circ}{m}\end{array}$ \\
\hline & 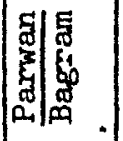 & $\begin{array}{lll}\infty & 0 & 0 \\
\dot{m} & \stackrel{0}{\sigma}\end{array}$ & $\leftarrow \infty$ & סי & $\stackrel{n}{\stackrel{n}{+}}$ & $\underset{\square}{\check{\sigma}}$ & 空 & $\stackrel{+}{\circ}$ & $\stackrel{\infty}{\infty}$ \\
\hline & 利热 & 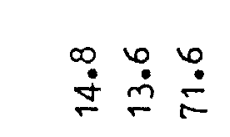 & $\begin{array}{l}\infty \\
\infty\end{array}$ & $\stackrel{N}{\mathscr{0}}$ & $\begin{array}{l}\dot{m} \\
\dot{m}\end{array}$ & $\stackrel{\llcorner}{\dddot{n}}$ & న్ & $\stackrel{0}{\infty}$ & 워 \\
\hline & 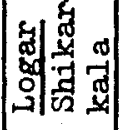 & $\stackrel{\circ}{\stackrel{\sim}{\sim}} \stackrel{\infty}{\stackrel{8}{\circ}}$ & $\ddot{\infty}$ & $\stackrel{0}{0}$ & $\stackrel{\infty}{\stackrel{0}{\prime}}$ & $\stackrel{\text { m }}{\dddot{\bullet}}$ & $\stackrel{\infty}{\circ}$ & $\stackrel{?}{\infty}$ & \& \\
\hline & F|c|c| & $\begin{array}{lll}\infty & 0 & 0 \\
\stackrel{i}{N} & \dot{0}\end{array}$ & $\stackrel{\text { in }}{\infty}$ & : & $\ddot{0}$ & $\stackrel{\text { ֻn }}{\check{0}}$ & $\stackrel{\circ}{2}$ & $\stackrel{+}{\circ}$ & $\underset{\sim}{\mathbb{N}}$ \\
\hline & 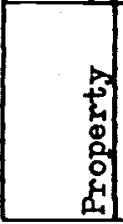 & 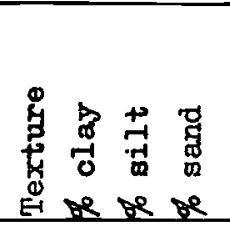 & 蜔 & 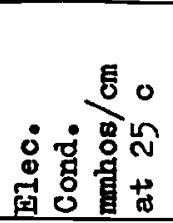 & 冬 & 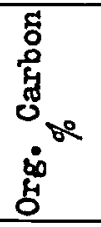 & 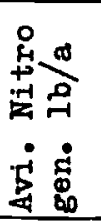 & 造密 & 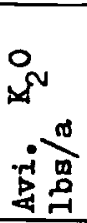 \\
\hline
\end{tabular}


APPEHDIX IIX

SOIL ANALYSIS DANA

Lagman Province:

(b) hingat $-2963-69$

\begin{tabular}{|c|c|c|c|c|c|c|c|}
\hline \multirow[t]{2}{*}{ S. No } & \multirow{2}{*}{ Villase } & \multirow{2}{*}{$\mathrm{pH}$} & \multirow{2}{*}{$\operatorname{Con} \dot{\alpha}$} & \multirow{2}{*}{$\mathrm{CaCO}_{3}$} & \multicolumn{3}{|c|}{ Nwalebie Futriente loofa } \\
\hline & & & & & Ii & $P$ & 1 \\
\hline 1 & Chelmatty & 7.5 & 0.50 & 1.63 & 175 & 11.2 & 200 \\
\hline 2 & Chelinatty & 3.0 & 0.45 & 1.38 & 200 & 9.0 & 395 \\
\hline 3 & Chelinatty & 8.0 & 0.64 & 1.76 & 120 & 7.6 & 23 \\
\hline 4 & Chelmatty & 7.8 & 0.50 & 1.50 & 336 & 6.4 & 180 \\
\hline 5 & Thendi & 8.5 & 0.50 & 5.50 & 420 & 8.0 & 135 \\
\hline 6 & Bazkhale & 8.2 & 0.45 & 2.13 & 420 & 0.0 & 140 \\
\hline 7 & Bazkhale & 8.3 & 0.50 & 2.25 & 100 & 0.0 & 120 \\
\hline 8 & Sheer Gar & 8.4 & 0.85 & 9.63 & 610 & $1 \% .2$ & $2 \div 0$ \\
\hline 9 & Sheer fort & 8.3 & 0.50 & 7.63 & 79 & $2, A$ & 250 \\
\hline 10 & Onaratai & 8.4 & 0.20 & 4.63 & 26 & 2.0 & 285 \\
\hline 11 & Goemer: & 8.4 & $0.2 ?$ & 2.3 & +0 & 2.2 & $x$ \\
\hline 12 & Demosien & $\Gamma \cdot 5$ & 0.40 & 1.25 & $\ldots$ & 10.0 & 7 \\
\hline 13 & Demosieri & 7.5 & 0.45 & 1.76 & $44 \hat{0}$ & 10.4 & 50 \\
\hline 14 & Nanakzai & 7.3 & 0.74 & 1.50 & 350 & 20.0 & 135 \\
\hline 15 & Pashea & $7 \cdot 5$ & 0.40 & 1.76 & 418 & 12.3 & 200 \\
\hline 16 & Alingar & 7.7 & 0.75 & 0.25 & 612 & 18.8 & 150 \\
\hline 17 & Alingar & 7.2 & 0.50 & 1.13 & 392 & 24.2 & 180 \\
\hline 18 & Chelmatty & 7.6 & 0.55 & 2.63 & 125 & 8.0 & 65 \\
\hline 19 & Sesadah. & 7.6 & 0.50 & 2.75 & 812 & 28.0 & 190 \\
\hline 20 & CheImatty: & 8.0 & 1.65 & 2.73 & 190 & 10.3 & 200 \\
\hline 21 & Chelmatty & 8.3 & 1.00 & 2.25 & 400 & 6.3 & 102 \\
\hline 22 & Chelmatty & 7.7 & 0.50 & 1.50 & 170 & 11.2 & 200 \\
\hline 23 & Che Imatty & 7.0 & 0.50 & 1.63 & 411 & 13.7 & 219 \\
\hline 24 & Chelmatty & 8.0 & 0.40 & 1.63 & 180 & 25.3 & 180 \\
\hline 25 & Chelmatty & 7.5 & 1.27 & 6.63 & 312 & 10.3 & 192 \\
\hline 26 & Alishang & 8.0 & 1.5 & 1.50 & 300 & 3.4 & 200 \\
\hline 27 & Hasanzar & 7.8 & 1.5 & 1.13 & 300 & 2.5 & 175 \\
\hline 28 & Alishang & 7.0 & 2.0 & 1.25 & 319 & 10.2 & 195 \\
\hline 29 & Tirgari & 7.8 & 0.25 & 1.25 & 411 & 6.1 & 70 \\
\hline 30 & Pashea & 8.0 & 0.45 & 0.88 & 172 & 6.5 & 87 \\
\hline 31 & Kanda & 7.8 & 0.45 & 1.50 & 462 & 8.0 & 50 \\
\hline 32 & Murderwar & 7.3 & 0.55 & 1.00 & 500 & 7.2 & 150 \\
\hline 33 & Agrabad & 7.6 & 0.50 & 1.13 & 432 & 6.5 & 310 \\
\hline 34 & Q. Sherullah & 8.0 & 0.95 & 1.63 & 420 & 22.8 & 411 \\
\hline 35 & Pashae & 8.2 & 0.80 & 2.5 & 462 & 23.5 & 178 \\
\hline
\end{tabular}


Lagnaan Province (continued)

\begin{tabular}{|c|l|c|c|c|c|c|c|}
\hline S.No. & Village & $\mathrm{pH}$ & Cond. & $\mathrm{CaCO}_{3}$ & \multicolumn{3}{|c|}{ Available Nutrients los/a } \\
\hline 36 & Murderwar & 8.6 & 0.90 & 0.88 & 448 & 4.2 & 200 \\
37 & Q. Kedce & 8.2 & 0.90 & 3.25 & 420 & 3.6 & 125 \\
38 & Q. Kazi & 7.9 & 0.50 & 0.63 & 350 & 4.5 & 60 \\
39 & Dehmyslem & 7.9 & 0.50 & 1.00 & 630 & 23.5 & 117 \\
40 & Kalatie & 8.1 & 0.80 & 1.75 & 128 & 11.5 & 129 \\
41 & Q. Syda & 7.2 & 0.60 & 1.25 & 200 & 10.3 & 200 \\
42 & Nagra & 8.2 & 0.60 & 1.00 & 175 & 3.4 & 120 \\
43 & Kanda & 7.1 & 1.30 & 1.13 & 218 & 4.6 & 150 \\
44 & Kanda & 8.1 & 0.90 & 6.38 & 476 & 3.7 & 101 \\
45 & Kanda & 7.9 & 0.72 & 0.0 & 387 & 2.5 & 97 \\
46 & Kanda & 7.3 & 2.1 & 0.88 & 150 & 5.2 & 213 \\
& & & & & & & \\
\hline
\end{tabular}

Nangarhar Province:

\begin{tabular}{|r|l|r|l|r|r|r|r|}
\hline 1 & Chappari Khail & 8.4 & 0.6 & 9.4 & 200 & 0.0 & 260 \\
2 & Chappari Khai & 8.3 & 0.7 & 10.63 & 250 & 0.0 & 340 \\
3 & Sangeen Kala & 9.2 & 0.8 & 14.5 & 400 & 1.3 & 370 \\
4 & Kasmabad & 8.4 & 0.7 & 8.25 & 100 & 10.1 & 160 \\
5 & Kasmabad & 8.0 & 1.3 & 11.75 & 358 & 0.0 & 375 \\
6 & Kasmabad & 8.0 & 1.3 & 9.75 & 200 & 11.2 & 370 \\
7 & Kasmabad & 7.5 & 0.7 & 6.50 & 170 & 10.4 & 260 \\
8 & Kasmabad & 7.9 & 0.6 & 7.75 & 180 & 10.4 & 340 \\
9 & K.Hakim Zaida & 8.0 & 0.6 & 12.63 & 210 & 0.0 & 417 \\
10 & Tan Wat & 8.5 & 0.4 & 12.87 & 179 & 0.0 & 250 \\
11 & Besood & 8.0 & 0.9 & 7.13 & 317 & 10.4 & 260 \\
12 & Kasmabad & 8.2 & 0.6 & 8.3 & 189 & 2.1 & 412
\end{tabular}


PaIris end Kapiad Province:

\begin{tabular}{|c|c|c|c|c|c|c|c|c|}
\hline S.No & Village & $\mathrm{pH}$ & Cond. & $\mathrm{CaOO}_{3}$ & \multicolumn{4}{|c|}{ AYridabla Nutrienth $1 \mathrm{ba} / \mathrm{a}$} \\
\hline & & & & & $N$ & $P$ & $K$ & \\
\hline 1 & Rahameni Lhyl & 8.0 & 0.6 & .63 & 200 & 10.4 & 400 & \\
\hline 2 & Rahamani Khyl & 7.0 & 0.5 & .37 & 175 & 4.0 & 400 & \\
\hline 3 & Rahamani Khyl & 8.4 & 1.1 & 3.62 & 572 & 12.0 & 500 & \\
\hline 4 & Ghasur Khyall & 7.2 & 1.0 & $1 . \infty$ & 300 & 6.4 & 270 & \\
\hline 5 & Togak & 8.0 & 1.5 & 0.63 & 317 & 10.2 & 340 & \\
\hline 6 & Ghafur Kryail & 7.2 & 0.45 & 0.25 & 322 & 10.0 & 400 & \\
\hline 7 & Dooba kili & 7.7 & 0.6 & 0.25 & 419 & 11.2 & 270 & \\
\hline 8 & Nawabad & 7.5 & 0.9 & 0.5 & 308 & 10.4 & 370 & \\
\hline 9 & Nawabad & 8.6 & 0.7 & 0.63 & 295 & 12.2 & 400 & \\
\hline 10 & Eenkochin & 8.2 & 1.1 & 3.62 & 299 & 16.2 & 130 & \\
\hline i) & Guei Bud & 8.3 & 0.7 & 3.38 & 350 & 24.7 & 200 & \\
\hline 2 & kejareho & 3.6 & 0.6 & 0.50 & 318 & 12.5 & 320 & \\
\hline 13 & Nejereno & 8.6 & 0.7 & 0.50 & 300 & 8.8 & 20 & \\
\hline is & Kaza Vher & 6.1 & 0.6 & $\therefore . \infty$ & 527 & 7.5 & 176 & \\
\hline 19 & Kaj-Sinali & 7.9 & 0.7 & 0.88 & 117 & 6.2 & 380 & \\
\hline 15 & Kak-Shal1 & 7.6 & 1.2 & 1.12 & 570 & 5.4 & 412 & \\
\hline 17 & Kalai Safed & 8.3 & 1.1 & 1.23 & 100 & 11.4 & 340 & \\
\hline 18 & ksoor Khyll & $7 \cdot 9$ & 1.7 & 2.25 & 312 & 3.8 & 390 & \\
\hline is & Asoor Khyll & 8.5 & 0.6 & 0.75 & 270 & 7.8 & 192 & - \\
\hline 20 & Asoor Khyll & 8.1 & 0.8 & 1.0 & 290 & 5.6 & 217 & \\
\hline 21 & Asoor Khyll & 8.0 & 1.1 & 5.4 & 300 & 6.7 & 422 & \\
\hline
\end{tabular}

\begin{tabular}{|l|l|l|l|r|r|r|r|}
\hline 1 & Chihutula & 8.0 & 0.35 & 10.0 & 238 & 8.0 & 200 \\
3 & Chasmansman & 8.2 & 0.25 & 2.5 & 210 & 11.3 & 300 \\
4 & Chasmansman & 8.1 & 1.3 & 12.6 & 300 & 6.4 & 310 \\
5 & Chijutula & 8.0 & 2.1 & 2.6 & 519 & 11.0 & 410 \\
6 & Chijutula & 8.2 & 1.3 & 1.9 & 208 & 3.8 & 360 \\
7 & Chijutula & 8.1 & 0.97 & 3.5 & 170 & 4.9 & 460 \\
8 & Tone Kohul & 8.0 & 0.33 & 11.8 & 317 & 5.7 & 370 \\
& Chimtula & 8.0 & 0.48 & 16.7 & 150 & 4.0 & 440 \\
\hline
\end{tabular}


Maidan:

\begin{tabular}{|c|c|c|c|c|c|c|c|}
\hline \multirow{2}{*}{ S.No. } & \multirow{2}{*}{ Village } & \multirow{2}{*}{$\mathrm{pH}$} & \multirow{2}{*}{ Cond. } & \multirow{2}{*}{$\mathrm{CaCO}_{3}$} & \multicolumn{3}{|c|}{ Available Nutrients Ibs/a } \\
\hline & & & & & N & $P$ & $X$ \\
\hline 1 & Shah Badin & 8.2 & 1.1 & 11.6 & 154 & 6.4 & 400 \\
\hline 2 & Ruhaman Khal & 8.0 & 0.6 & 5.4 & 100 & 23.7 & 650 \\
\hline 3 & Mullahkail & 8.2 & 0.45 & $7 \cdot 1$ & 97 & 11.2 & 417 \\
\hline 4 & D.Shirin Dil & 8.5 & 1.0 & 15.8 & 125 & 3.6 & 375 \\
\hline 5 & Sepataw & 8.0 & 0.6 & 16.7 & 270 & 12.6 & 200 \\
\hline 6 & Smell Khial & 8.3 & 0.45 & 7.4 & 190 & $4 \cdot 3$ & 390 \\
\hline 7 & Mullakhail & 8.1 & 0.8 & $12 \cdot 2$ & 300 & 0.0 & 419 \\
\hline 8 & Bullande & 8.1 & 0.5 & 11.2 & 278 & 1.7 & 425 \\
\hline 9 & Bullande & 8.2 & 0.8 & 14.6 & 70 & 2.8 & 216 \\
\hline 10 & Busarek & 8.4 & 1.0 & 12.6 & 60 & 3.5 & 180 \\
\hline
\end{tabular}

Logar Province:

\begin{tabular}{|c|c|c|c|c|c|c|c|}
\hline 1 & Deh Sheikh & 8.2 & 0.75 & 20.5 & 475 & 10.4 & 625 \\
\hline 2 & Jai Shaxha & 8.4 & 0.55 & $22 \cdot 5$ & 400 & 8.0 & 400 \\
\hline 3 & Q Baba Pathkan & 8.0 & 1.7 & 21.8 & 380 & 2.4 & 375 \\
\hline 4 & Pamakwa Rongi & 7.9 & 1.9 & 20.6 & 417 & 6.4 & 500 \\
\hline 5 & Q Das Shal & 8.1 & 0.7 & 21.2 & 300 & 2.4 & 490 \\
\hline 6 & Q Das Shal & 8.2 & 0.65 & 16.0 & 312 & 10.4 & 620 \\
\hline 7 & Q Babi & 8.0 & 0.4 & 15.6 & 170 & 3.0 & 519 \\
\hline 8 & Pullailum & 8.2 & 0.7 & 24.0 & 70 & 4.0 & 416 \\
\hline 9 & Pullailum & 8.0 & 0.8 & 17.5 & 60 & 5.0 & 400 \\
\hline 10 & Deh Shakh & $7 \cdot 9$ & 1.5 & 21.0 & 100 & 11.3 & 390 \\
\hline 11 & Paydeen & 8.5 & 0.6 & 16.2 & 300 & 0.5 & 400 \\
\hline 12 & Deh Shakhi & 8.4 & 0.75 & $17 \cdot 9$ & 270 & 1.7 & 412 \\
\hline 13 & Deh Shaikhi & 8.0 & 1.5 & 15.2 & 316 & 4.2 & 330 \\
\hline
\end{tabular}


Kabul Provinco:

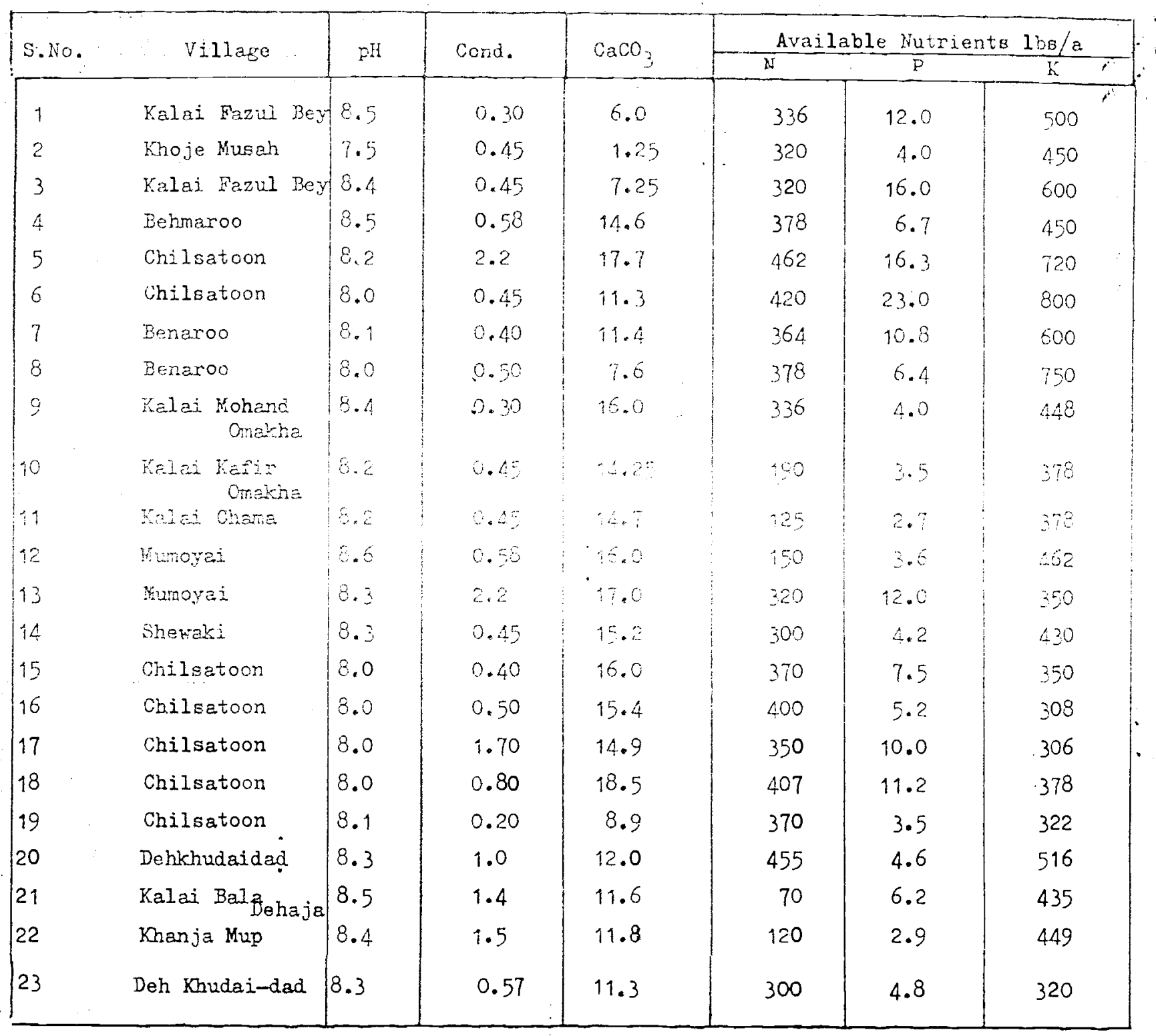




\section{APPENDIX IJII}

SOIL ANALYSIS DATA

(c) Wheat - $1969-1270$

\begin{tabular}{|c|c|c|c|c|c|c|c|c|c|}
\hline \multirow{2}{*}{$\begin{array}{l}\text { Province } \\
\text { s.no. }\end{array}$} & \multicolumn{3}{|c|}{ Texture } & \multirow{2}{*}{$\begin{array}{r}\mathrm{pH} \\
1: 5\end{array}$} & \multirow{2}{*}{$\begin{array}{l}\text { Cond } \\
\mathrm{mm} / 25^{\circ} \mathrm{C}\end{array}$} & \multirow{2}{*}{$\begin{array}{c}\mathrm{CaCO}_{3} \\
8 \\
\end{array}$} & \multirow{2}{*}{$\begin{array}{c}\text { O. Carbon } \\
.8\end{array}$} & \multicolumn{2}{|c|}{ Available } \\
\hline & $\begin{array}{c}\text { Clay } \\
\%\end{array}$ & $\begin{array}{c}\text { Silt } \\
\%\end{array}$ & $\begin{array}{c}\text { Sand } \\
0 \\
\end{array}$ & & & & & $\mathrm{Plb} / \mathrm{a}$ & $\mathrm{K}_{2} \mathrm{O} \mathrm{lb} / \mathrm{a}$ \\
\hline Kabul 1 & 32 & 42.0 & 26.0 & 8.5 & 0.20 & 5.25 & 0.46 & 8.5 & 155 \\
\hline 2 & 29.2 & 31.6 & 39.2 & 8.5 & 0.13 & 6.20 & 0.39 & 17.0 & 220 \\
\hline 3 & 20.0 & 32.0 & 48.0 & 8.5 & 0.01 & 5.50 & 0.65 & 4.75 & 350 \\
\hline 4 & 22.0 & 32.0 & 46.0 & 8.7 & 0.07 & 5.75 & 0.34 & 28.0 & 135 \\
\hline 5 & 28.0 & 44.0 & 28.0 & 8.7 & 0.02 & 8.50 & 0.47 & 28.0 & 295 \\
\hline 6 & - & - & - & - & - & - & - & - & - \\
\hline 7 & 22.0 & 46.0 & 32.0 & 8.5 & 0.05 & 11.25 & 0.42 & 19.26 & 295 \\
\hline 8 & 26.0 & 42.0 & 32.0 & 8.6 & 0.04 & 10.75 & 0.39 & 11.2 & 400 \\
\hline 9 & 26.0 & 42.0 & 32.0 & 8.8 & 0.05 & 8.75 & 0.18 & 7.4 & 395 \\
\hline 10 & 40.0 & 34.0 & 26.0 & 8.7 & 0.04 & 13.00 & 0.72 & 4.8 & 310 \\
\hline 11 & 22.0 & 30.0 & 48.0 & 8.5 & 0.05 & 15.00 & 0.29 & 11.50 & 185 \\
\hline 12 & 23.0 & 38.0 & 40.0 & 8.3 & 0.01 & 3.00 & 0.65 & 20.50 & 235 \\
\hline 13 & 24.0 & 36.0 & 40.0 & 8.4 & 0.06 & 3.90 & 0.24 & 2.75 & 220 \\
\hline 14 & 18.0 & 46.0 & 36.0 & 8.3 & 0.01 & 8.25 & 0.35 & 7.50 & 120. \\
\hline 15 & 24.0 & 40.0 & 36.0 & 8.1 & 0.01 & 3.50 & 0.75 & 5.50. & 295 \\
\hline 16 & 22.0 & 44.0 & 34.0 & 8.1 & 0.04 & 3.25 & 0.55 & 20.0 & 150 \\
\hline 17 & 20.0 & 46.0 & 34.0 & 8.2 & 0.01 & 12.50 & 0.65 & 6.3 & 350 \\
\hline 18 & 11.2 & 31.6 & 57.2 & 8.2 & 0.05 & 12.25 & 0.28 & 11.50 & - \\
\hline 19 & 28.2 & 46.0 & 26.0 & 8.3 & - & 6.25 & 0.48 & 13.0 & 200 \\
\hline 20 & 16.0 & 34.0 & 50.0 & 8.5 & 0.02 & 8.25 & 0.30 & 3.00 & 140 \\
\hline 21 & 16.0 & 40.0 & 56.0 & 8.5 & 0.03 & 5.25 & 0.60 & 2.50 & 240 \\
\hline 22 & 20.0 & 32.0 & 48.0 & 8.2 & 0.28 & 3.00 & 0.35 & 3.89 & 270 \\
\hline 23 & 18.0 & 36.0 & 46.0 & 8.3 & 0.30 & 17.5 & 0.69 & 1.50 & 180 \\
\hline 24 & 22.0 & 42.0 & 36.0 & 8.2 & 0.03 & 17.50 & 0.69 & 3.62 & 200 \\
\hline 25 & 23.2 & 41.6 & 35.0 & 8.1 & 0.22 & 17.50 & 0.24 & 17.5 & 150 \\
\hline 26 & 29.2 & 38.0 & 32.8 & 8.3 & 0.07 & 18.00 & 0.60 & 2.7 & 375 \\
\hline 27 & 26.0 & 42.0 & 32.0 & 8.2 & 0.04 & 10.8 & 0.27 & 2.7 & 355 \\
\hline 28 & 20.0 & 50.0 & 30 & 8.5 & 0.06 & 18.25 & 0.70 & 20.0 & 355 \\
\hline 29 & 23.2 & 27.6 & 49.2 & 8.0 & 0.02 & 18.75 & 0.62 & 12.8 & 190 \\
\hline 30 & 23.2 & 39.6 & 37.2 & 8.7 & 0.03 & 11.23 & 0.40 & 8.2 & 275 \\
\hline 31 & 32.0 & 34.0 & 34.0 & 8.1 & 0.03 & 19.0 & 0.45 & 7.50 & 150 \\
\hline 32 & 30.0 & 42.0 & 28.0 & 8.2 & 0.02 & 14.5 & 0.55 & 1.72 & 395 \\
\hline
\end{tabular}




\begin{tabular}{|c|c|c|c|c|c|c|c|c|c|}
\hline \multirow{2}{*}{$\begin{array}{l}\text { Province } \\
\text { a.mo. }\end{array}$} & \multicolumn{3}{|c|}{ Toxturo } & \multirow{2}{*}{$\begin{array}{l}\mathrm{pH} \\
1: 5\end{array}$} & \multirow{2}{*}{ cond. } & \multirow{2}{*}{$\begin{array}{r}\mathrm{CaCO}_{3} \\
. \\
\end{array}$} & \multirow{2}{*}{$\begin{array}{c}0 . \text { Cerbon } \\
\end{array}$} & \multicolumn{2}{|c|}{ Avajlable } \\
\hline & $\begin{array}{r}c 13 y \\
y\end{array}$ & $\begin{array}{c}\text { Silt } \\
0 f \\
\end{array}$ & $\begin{array}{c}\text { Sand } \\
g\end{array}$ & & & & & $\mathrm{P} 1 \mathrm{~b} / \mathrm{a}$ & $\mathrm{K}_{2} \mathrm{O} \mathrm{Ib} / \mathrm{a}$ \\
\hline 33 & - & - & - & - & - & - & - & - & 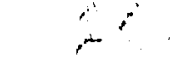 \\
\hline 34 & 28.0 & 44.0 & 28.0 & 8.0 & 0.04 & 21.0 & 0.28 & 20.50 & 410 \\
\hline 35 & 28.0 & 46.0 & 26.0 & 8.2 & 0.03 & 14.0 & 0.10 & 8.50 & 370 \\
\hline 36 & 27.0 & 45.0 & 28.0 & 8.0 & 0.20 & 10.50 & 0.75 & 8.50 & 300 \\
\hline 37 & 26 & 38 & 36 & 8.2 & 0.02 & 16.40 & 0.68 & 15.50 & 355 \\
\hline 38 & 28 & 38 & 34 & 8.4 & 0.10 & 17.00 & 0.10 & 12.10 & 245 \\
\hline 39 & 26 & 44 & 30 & 8.7 & 0.13 & 17.40 & 0.75 & 15.50 & 195 \\
\hline 40 & 22 & 38 & 40 & 8.2 & 0.09 & 18.40 & 0.69 & 15.50 & 370 \\
\hline 42 & 32 & 38 & 30 & 8.3 & 0.03 & 10.75 & 0.34 & 8.50 & 145 \\
\hline 42 & 28 & 38 & 34 & $8.5^{\circ}$ & 0.30 & 3.75 & 0.63 & 27.0 & 125 \\
\hline 43 & 22 & 44 & 34 & 8.8 & 0.20 & 7.50 & 0.42 & 0.20 & 325 \\
\hline 44 & 19.2 & 22.6 & 29.2 & 8.7 & 0.04 & 25.0 & 0.22 & 0.50 & $i_{r}=5$ \\
\hline 45 & 24 & 42 & 34 & 8.5 & 0.40 & 25.0 & 0.22 & $i, \infty$ & $32=$ \\
\hline 46 & 20 & 44 & 36 & 8.3 & 0.40 & 14.75 & 0.47 & 3.00 & 290 \\
\hline 47 & 24 & 42 & 34 & 8.4 & 0.28 & 13.00 & 0.49 & 2.80 & 258 \\
\hline 48 & 26 & 38 & 36 & 8.2 & 0.02 & 14.80 & 0.12 & 11.50 & 4.75 \\
\hline 49 & 22 & 42 & 36 & 8.8 & 0.10 & 15.00 & 0.15 & 15.50 & 360 \\
\hline 50 & 28 & 44 & 28 & 8.2 & 0.10 & 15.20 & 0.12 & 17.50 & 355. \\
\hline 51 & 28 & 44 & 28 & 8.3 & 0.20 & 15.20 & 0.42 & 5.50 & $205^{\circ}$ \\
\hline 52 & 20 & 46 & 34 & 8.0 & 0.02 & 18.20 & 0.42 & 20.0 & 210 \\
\hline 53 & 24 & 40. & 36 & 8.2 & 0.40 & 16.00 & 0.19 & 14.0 & 195 \\
\hline 54 & - & 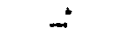 & - & - & - & - & - & - & - \\
\hline 55 & 22 & 30 & 48 & 8.3 & 0.06 & 15.00 & 0.10 & 16.00 & 285 \\
\hline 56 & 24 & 44 & 32 & 8.5 & 0.35 & 8.5 & 0.82 & 2.75 & 425 \\
\hline 57 & 14 & 22 & 64 & 8.2 & 0.36 & 7.5 & 0.69 & 1.70 & 210 \\
\hline 58 & 16 & 44 & 40 & 8.0 & 0.11 & 10.2 & 0.65 & 4.00 & 265 \\
\hline $\begin{array}{r}\text { Ghazni } \\
1\end{array}$ & 29.4 & 9.6 & 62.0 & 8.7 & 0.20 & 16.75 & 0.40 & 8.2 & 315 \\
\hline 2 & 30.6 & 20.8 & 48.8 & 8.5 & 0.19 & 15.75 & 0.57 & 2.7 & 360 \\
\hline 3 & 27.2 & 5.6 & 67.2 & 8.5 & 0.30 & 12.00 & 0.25 & 3.5 & 190 \\
\hline 4 & 27.2 & 5.6 & 67.2 & 8.7 & 0.18 & 15.50 & 0.30 & 4.6 & 150 \\
\hline 5 & 36.4 & 19.6 & 44.0 & 8.9 & 0.22 & 12.50 & 0.49 & 7.6 & 270 \\
\hline 6 & 30.4 & 7.6 & 62.0 & 8.5 & 0.19 & 15.25 & 0.38 & 4.4 & 275 \\
\hline 7 & 30.4 & 5.6 & 64.0 & 8.5 & 0.17 & 11.25 & 0.26 & 3.9 & 190 \\
\hline 8. & 30.4 & 9.6 & 60 & 8.5 & 0.20 & 13.25 & 0.48 & 6.7 & 145 \\
\hline
\end{tabular}




\begin{tabular}{|c|c|c|c|c|c|c|c|c|c|}
\hline \multirow{2}{*}{$\begin{array}{l}\text { Provinoe } \\
\text { s.no. }\end{array}$} & \multicolumn{3}{|c|}{ Texture } & \multirow{2}{*}{$\begin{array}{l}\mathrm{pH} \\
1: 5\end{array}$} & \multirow{2}{*}{$\begin{array}{l}\text { Cond. } \\
\mathrm{mm} / 25^{\circ} \mathrm{c}\end{array}$} & \multirow{2}{*}{$\begin{array}{r}\mathrm{CaCO}_{3} \\
.8 \\
\end{array}$} & \multirow{2}{*}{$\begin{array}{c}\text { O. Carbon } \\
\% \\
\end{array}$} & \multicolumn{2}{|c|}{ Available } \\
\hline & $\begin{array}{c}\text { Clay } \\
0 \\
\end{array}$ & $\begin{array}{c}\text { Silt } \\
0\end{array}$ & $\begin{array}{c}\text { Sand } \\
\%\end{array}$ & & & & & $P 1 b / a$ & $01 b / a$ \\
\hline Onazni & & & & & & & & & $\therefore$ \\
\hline 9 & 36.4 & 5.6 & 58 & 8.6 & 0.31 & 12.50 & 0.42 & 7.4 & 245 \\
\hline 10 & 38.4 & 19.6 & 42 & 8.6 & 0.30 & $11: 75$ & 0.59 & 3.6 & 295 \\
\hline 11 & 35.2 & 17.6 & 47.2 & 8.5 & 0.29 & 14.25 & 0.42 & 4.8 & 175 \\
\hline 12 & 40.4 & 11.6 & 48.0 & 8.4 & 0.26 & 5.25 & 0.40 & 8.8 & 175 \\
\hline 13 & 42.4 & 17.6 & 40.0 & 8.6 & 0.31 & 3.75 & 0.50 & 7.8 & 245 \\
\hline 14 & 18.4 & 11.6 & 70.0 & 8.4 & 0.32 & 11.75 & 0.40 & 6.7 & 145 \\
\hline 15 & 24.4 & 11.6 & 64.0 & 8.5 & 0.36 & $14 \cdot 50$ & 0.10 & 7.9 & 355 \\
\hline 16 & 36.4 & 21.6 & 42.0 & 8.3 & 0.42 & 14.75 & 0.50 & 8.0 & 355 \\
\hline 17 & 32.6 & 9.6 & 58.0 & 8.0 & 0.40 & 16.50 & 0.62 & 7.9 & 185 \\
\hline 18 & 42.4 & 17.6 & 40.0 & 8.5 & 0.37 & 13.25 & 0.41 & 8.0 & 525 \\
\hline 1 & 14 & 34 & 32 & 7.9 & I.I & 10.5 & 10.5 & 0.65 & 160 \\
\hline 2 & 12 & 36 & 52 & 8.2 & $1 . i$ & 9.3 & 0.41 & 4.0 & 150 \\
\hline 3 & 12 & 40 & 48 & 8.0 & 1.1 & 10.3 & 0.43 & 8.5 & 165 \\
\hline 4 & 12 & 32 & 56 & 8.0 & 1.1 & 9.3 & 0.33 & 5.3 & 200 \\
\hline 5 & 10 & 38 & 52 & 7.9 & 1.1 & 10.5 & 0.33 & 2.6 & 245 \\
\hline 6 & 16 & 32 & 52 & 8.2 & 1.0 & 6.0 & 0.50 & 11.5 & $80 \ldots$ \\
\hline 7 & 10 & 38 & 52 & 8.0 & 1.3 & 10.0 & 0.42 & 3.6 & 200 \\
\hline 8 & 10 & 36 & 54 & 8.1 & 1.2 & 8.3 & 0.41 & 1.4 & 160 \\
\hline 9 & 12 & 36 & 52 & 8.2 & 1.2 & 9.5 & 0.38 & 4.4 & 190 \\
\hline 10 & 12 & 36 & 52 & 7.9 & 1.3 & 10.5 & 0.50 & 2.3 & 225 \\
\hline 11 & 14 & 32 & 54 & 8.0 & 2.1 & 10.3 & 0.13 & 2.6 & 260 \\
\hline 12 & 14 & 32 & 54 & 8.0 & 1.3 & 9.7 & 0.51 & 2.5 & 175 \\
\hline 13 & 14 & 34 & 52 & 8.0 & 1.3 & 10.3 & 0.30 & 3.2 & 235 \\
\hline 14 & 14 & 32 & 54 & 7.9 & 1.2 & 9.0 & 0.40 & 6.5 & 225 \\
\hline 15 & 14 & 34 & 52 & 7.8 & 1.6 & 8.7 & 0.50 & 2.2 & 170 \\
\hline 16 & 14 & 32 & 54 & 8.0 & 1.3 & 10.0 & 0.28 & 4.6 & 225 \\
\hline 17 & 12 & 36 & 52 & 8.0 & 1.0 & 9.7 & 0.50 & 2.8 & 200 \\
\hline 18 & 14 & 36 & 50 & 8.0 & 1.0 & 10.5 & 0.37 & 1.6 & 285 \\
\hline 19 & 12 & 34 & 54 & 8.1 & 0.9 & 9.0 & 0.50 & 4.0 & 200 \\
\hline 20 & 12 & 36 & 52 & 8.4 & 0.8 & 8.7 & 0.30 & 4.0 & 155 \\
\hline 21 & 38 & 28 & 42 & 8.5 & 0.5 & 2.5 & 0.20 & 3.8 & 196 \\
\hline 22 & - & - & - & - & - & - & - & - & - \\
\hline 23 & 30 & 8 & 62 & 8.4 & 0.8 & 3.3 & 0.12 & 10.5 & 205 \\
\hline 24 & - & - & - & - & - & - & - & - & - \\
\hline
\end{tabular}




\begin{tabular}{|c|c|c|c|c|c|c|c|c|c|}
\hline \multirow{2}{*}{$\begin{array}{l}\text { Province } \\
\text { s.no. }\end{array}$} & \multicolumn{3}{|c|}{ Texture } & \multirow{2}{*}{$\begin{array}{r}p \mathrm{pi} \\
1: 5\end{array}$} & \multirow{2}{*}{ Cond. } & \multirow{2}{*}{$\mathrm{CaCO}_{3}$} & \multirow{2}{*}{$\begin{array}{l}\text { 0. Carbon } \\
\text { of }\end{array}$} & \multicolumn{2}{|c|}{ Available } \\
\hline & 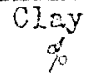 & $\begin{array}{c}5 i 2 t \\
6\end{array}$ & $\underset{d x}{\operatorname{Sand}}$ & & & & & $\mathrm{Plb} / \mathrm{a}$ & $K_{2} O \quad l b / a$ \\
\hline 25 & 32 & 24 & 44 & 3.4 & 0.5 & 3.1 & 0.32 & $4 \cdot 3$ & $190^{\circ}$ \\
\hline 26 & 21 & 11 & 68 & 8.4 & 0.2 & 3.0 & 0.15 & 5.8 & 148 \\
\hline 27 & 31 & 24 & 45 & 8.3 & 0.3 & 2.1 & 0.31 & 8.4 & 115 \\
\hline 28 & - & - & - & - & - & - & - & - & - \\
\hline 29 & 38 & 17 & 45 & 8.2 & 0.5 & 2.9 & 0.23 & 3.9 & 139 \\
\hline 30 & 22 & 8 & 70 & 7.8 & 1.2 & 3.3 & 0.92 & 1.5 & 255 \\
\hline 32 & 23 & 9 & 68 & 8.0 & 1.1 & 2.5 & 0.44 & 14.0 & 390 \\
\hline 32 & 26 & 26 & 58 & 8.2 & 1.3 & 12.8 & 0.87 & 2.5 & 115 \\
\hline 33 & 38 & 18 & 44 & 7.5 & 0.5 & 12.5 & 0.60 & 5.5 & 300 \\
\hline $3 A$ & 32 & 13 & 56 & 7.8 & 0.3 & 5.4 & 0.50 & 2.8 & 200 \\
\hline 35 & 35 & $\mathrm{~g}$ & 66 & $E .2$ & $\therefore=$ & $\therefore 5$ & 0.20 & $\therefore 0$ & 245 \\
\hline 36 & - & - & - & - & $\cdots$ & - & - & - & - \\
\hline 37 & 22 & 21 & $E T$ & 8.1 & 6.5 & 7.0 & 0.37 & $\therefore 0$ & 225 \\
\hline 38 & 20 & 37 & 43 & 0.2 & 0.7 & 1.9 & 0.43 & 0.5 & 215 \\
\hline 39 & 36 & 21 & 43 & 8.0 & 0.9 & 3.5 & 0.35 & 4.0 & 190 \\
\hline 40 & 22 & 11 & 67 & 8.3 & 2.0 & 4.5 & 0.42 & 6.5 & 100 \\
\hline Logar & 26 & 42 & 32 & 8.4 & 0.19 & 19.5 & 0.12 & 8.5 & 115 \\
\hline 2 & 28 & 44 & 28 & 8.3 & 0.25 & 22.3 & 0.32 & 6.1 & 200 \\
\hline 3 & 26 & 46 & 28 & 8.3 & 0.20 & 17.3 & 0.42 & 2.6 & 140 \\
\hline 4 & 32 & 36. & 32 & 8.4 & 0.13 & 19.2 & 0.10 & 3.9 & 165 \\
\hline 5 & 24 & 44 & 32 & 8.9 & 0.11 & 20.5 & 0.50 & 4.1 & 145 \\
\hline 6 & 40 & 39 & 21 & 8.6 & 0.12 & 18.0 & 0.37 & 3.5 & 130 \\
\hline 7 & 40 & 36 & 24 & 8.2 & 0.48 & 19.8 & 0.27 & 1.5 & 425 \\
\hline 8 & 32 & 42 & 26 & 8.2 & 0.19 & 19.3 & 0.50 & 3.0 & 85 \\
\hline 9 & 20 & 56 & 24 & 8.5 & 0.14 & 19.3 & 0.45 & 5.3 & 155 \\
\hline 10 & 30 & 46 & 24 & 8.4 & 0.12 & 19.0 & 0.46 & 4.6 & 155 \\
\hline 11 & 32 & 42 & 26 & 8.9 & 0.14 & 19.5 & 0.48 & 3.0 & 50 \\
\hline 12 & 32 & 46 & 22 & 8.6 & 0.12 & 21.0 & 0.39 & 6.5 & 85 \\
\hline 13 & 28 & 48 & 24 & 8.8 & 0.30 & 14.0 & 0.10 & 1.4 & 185 \\
\hline 14 & 28 & 44 & 28 & 8.5 & 0.14 & 19.0 & 0.48 & 1.5 & 115 \\
\hline 15 & 28 & 46 & 26 & 8.4 & 0.13 & 21.0 & 0.27 & 2.8 & 225 \\
\hline 16 & 18 & 48 & 34 & 8.5 & 0.18 & 18.3 & 0.10 & 3.0 & 130 \\
\hline 17 & 30 & 42 & 28 & 8.3 & 0.30 & 15.0 & 0.44 & 2.0 & 155 \\
\hline 18 & 24 & 42 & 34 & 8.7 & 0.19 & 15.5 & 0.12 & 2.5 & 230 \\
\hline 19 & 30 & 46 & 24 & 8.3 & 0.18 & 12.3 & 0.14 & 1.4 & 195.0 \\
\hline
\end{tabular}




\begin{tabular}{|c|c|c|c|c|c|c|c|c|c|}
\hline \multirow{2}{*}{$\begin{array}{c}\text { Province } \\
\text { s. no. }\end{array}$} & \multicolumn{3}{|c|}{ Texture } & \multirow{2}{*}{$\begin{array}{l}\mathrm{pH} \\
1: 5\end{array}$} & \multirow{2}{*}{$\begin{array}{l}\text { Cond. } \\
\mathrm{mm} / 25^{\circ} \mathrm{c}\end{array}$} & \multirow{2}{*}{$\begin{array}{c}\mathrm{CaCO}_{3} \\
\not 0\end{array}$} & \multirow{2}{*}{$\begin{array}{c}0 . \text { Carbon } \\
\end{array}$} & \multicolumn{2}{|c|}{ Available } \\
\hline & $\begin{array}{c}\mathrm{Clay} \\
\%\end{array}$ & $\begin{array}{c}\text { Silt } \\
\%\end{array}$ & $\begin{array}{r}\text { Sand } \\
\%\end{array}$ & & & & & $\mathrm{Plb} / \mathrm{a}$ & $\mathrm{K}_{2} 01 \mathrm{~b} / \mathrm{a}$ \\
\hline${ }_{20}^{\text {Logar }_{20}}$ & 34 & 44 & 22 & 8.4 & 0.14 & 22.5 & 0.13 & 2.3 & 205.0 \\
\hline 21 & 30 & 40 & 30 & 8.5 & 0.13 & 21.5 & 0.10 & 3.2 & 130.0 \\
\hline 22 & 26 & 48 & 26 & 7.8 & 0.16 & 21.4 & 0.43 & 3.0 & 160.0 \\
\hline 23 & 24 & 46 & 30 & 8.7 & 0.12 & 20.0 & 0.12 & 8.5 & 270.0 \\
\hline 24 & 34 & 40 & 26 & 7.8 & 0.11 & 23.0 & 0.50 & 10.50 & 205.0 \\
\hline 25 & 20 & 28 & 52 & 8.6 & 0.11 & 19.0 & 0.44 & 5.3 & 100.0 \\
\hline 26 & 26 & 44 & 30 & 8.7 & 0.13 & 16.7 & 0.27 & 1.30 & 135.0 \\
\hline 27 & 30 & 42 & 28 & 8.5 & 0.11 & 15.5 & 0.27 & 9.0 & 130.0 \\
\hline 28 & 38 & 42 & 20 & 8.6 & 0.12 & 17.2 & 0.32 & 4.1 & 175.0 \\
\hline 29 & 40 & 36 & 24 & 8.5 & 0.12 & 20.2 & 0.57 & 2.6 & 145.0 \\
\hline 30 & 30 & 40 & 30 & 8.3 & 0.14 & 18.00 & 0.44 & 7.5 & 155.0 \\
\hline 31 & 32 & 40 & 28 & 8.8 & 0.12 & 20.0 & 0.27 & 8.5 & 290 \\
\hline 32 & 32 & 46 & 22 & 8.5 & 0.12 & 21.0 & 0.26 & 6.2 & 175 \\
\hline 33 & 30 & 38 & 32 & 8.4 & 0.13 & 18.5 & 0.40 & 5.6 & 140 \\
\hline 34 & 26 & 40 & 34 & 8.6 & 0.16 & 22.7 & 0.26 & 8.2 & 150 \\
\hline 35 & 26 & 44 & 30 & 8.5 & 0.19 & 19.7 & 0.13 & 10.2 & 185 \\
\hline 36 & 32 & 48 & 20 & 8.7 & 0.15 & 16.0 & 0.50 & 4.4 & 195 \\
\hline 37 & 32 & 40 & 28 & 8.2 & 0.19 & 19.9 & 0.12 & 8.5 & $160^{\circ}$ \\
\hline 38 & 34 & 38 & 28 & 8.1 & 0.14 & 18.7 & 0.50 & 7.1 & 270 \\
\hline 39 & 24 & 44 & 32 & 8.4 & 0.16 & 19.5 & 0.40 & 5.6 & 130 \\
\hline 40 & 30 & 48 & 23 & 8.3 & 0.11 & 23.5 & 0.16 & 1.7 & 195 \\
\hline 41 & 34 & 42 & 24 & 8.5 & 0.12 & 20.8 & 0.62 & 2.4 & 135 \\
\hline 42 & 26 & 44 & 30 & 8.2 & 0.12 & 15.5 & 0.11 & 4.4 & 110 \\
\hline 43 & 30 & 46 & 24 & 8.8 & 0.13 & 17.8 & 0.38 & 6.2 & 170 \\
\hline 44 & 30 & 44 & 26 & 8.3 & 0.13 & 18.5 & 0.42 & 7.0 & 165 \\
\hline 45 & 24 & 36 & 40 & 8.7 & 0.15 & 12.8 & 0.50 & 8.8 & 165 \\
\hline${ }_{1}^{\text {Maidan }}$ & 30 & 43 & 27 & 8.6 & 0.30 & 9.3 & 0.50 & 1.3 & 220 \\
\hline 2 & 20 & 33 & 47 & 8.5 & 0.35 & - & 0.35 & 2.3 & 240 \\
\hline 3 & 26 & 39 & 35 & 8.7 & 0.30 & 9.2 & 0.25 & 5.5 & 110 \\
\hline 4 & 22 & 28 & 50 & 8.6 & 0.35 & 5.2 & 0.42 & 9.5 & 145 \\
\hline 5 & 26 & 37 & 37 & 8.4 & 0.25 & 6.7 & 0.47 & - & 160 \\
\hline 6 & 26 & 38 & 36 & 8.2 & 0.90 & 16.0 & 0.30 & 2.1 & 550 \\
\hline 7 & 28 & 33 & 39 & 8.7 & 0.55 & 17.3 & 0.15 & 4.0 & 295 \\
\hline 8 & 27 & 38 & 35 & 8.4 & 0.38 & 6.1 & 0.37 & 3.50 & 200 \\
\hline
\end{tabular}




\begin{tabular}{|c|c|c|c|c|c|c|c|c|c|}
\hline \multirow{2}{*}{$\begin{array}{l}\text { Province } \\
\text { s. no. }\end{array}$} & \multicolumn{3}{|c|}{ Tezture } & \multirow{2}{*}{$\begin{array}{l}\mathrm{pl} \\
1: 5\end{array}$} & \multirow{2}{*}{$\begin{array}{l}\text { Conda } \\
\mathrm{mm} / 25^{\circ} \mathrm{c}\end{array}$} & \multirow{2}{*}{$\begin{array}{c}\mathrm{CaCO}_{3} \\
. \\
\end{array}$} & \multirow{2}{*}{$\begin{array}{c}0 . \text { Carbon } \\
0\end{array}$} & \multicolumn{2}{|c|}{ Available } \\
\hline & $\begin{array}{c}\text { Clay } \\
\%\end{array}$ & $\begin{array}{c}\text { Silt } \\
\%\end{array}$ & $\begin{array}{c}\operatorname{San} \alpha \\
\%\end{array}$ & & & & & $\mathrm{P} 1 \mathrm{~b} / \mathrm{a}$ & $\mathrm{K}_{2} \mathrm{O} \mathrm{lb} / \mathrm{a}$ \\
\hline $\begin{array}{r}\text { Maidan } \\
9\end{array}$ & 25 & 43 & 36 & 8.4 & 0.38 & 4.2 & 0.28 & - & 200 \\
\hline 10 & 30 & 43 & 27 & 8.5 & 0.38 & 27.7. & 0.25 & 9.5 & 550 \\
\hline 11 & 21 & 38 & 41 & 8.1 & 0.80 & 15.8. & 0.14 & 2.3 & 290 \\
\hline 12 & 25 & 39 & 36 & 8.0 & 0.20 & 18.2 & 0.65 & 8.5 & 285 \\
\hline 13 & 22 & 44 & 34 & 8.2 & 0.45 & 11.9 & 0.40 & 9.5 & 250 \\
\hline 14 & 29 & 36 & 35 & 8.5 & 0.50 & 5.9 & 0.48 & 3.5 & 415 \\
\hline 15 & 28 & 44 & 28 & 8.4 & 0.50 & 6.1 & 0.47 & 1.8 & 235 \\
\hline 16 & 17 & 28 & 55 & 8.5 & 0.50 & 5.8 & 0.22 & 5.50 & 260 \\
\hline 17 & - & - & - & - & - & - & - & - & - \\
\hline 18 & 28 & 29 & 43 & 8.3 & 0.50 & 8.1 & 0.40 & 3.0 & 260 \\
\hline 19 & 27 & 31 & 42 & 8.2 & 0.50 & 7.2 & 0.25 & 2.1 & 600 \\
\hline 20 & 20 & 15 & 75 & S.4 & 0.50 & $2 \pi . ?$ & 0.46 & 6.1 & $2:$ \\
\hline 21 & 26 & $\angle 2$ & 33 & 3.5 & 2.6 & 10.6 & 0.2 & $\therefore .0$ & 750 \\
\hline 22 & 27 & 39 & 36 & 8.6 & 0.90 & 220 & 0.50 & 8.5 & 27 \\
\hline 23 & 16 & 25 & 59 & 8.5 & 0.70 & 2.3 & 0.65 & 6.50 & 320 \\
\hline 24 & - & - & - & - & - & - & - & - & - \\
\hline 25 & 26 & 43 & 31 & 8.5 & 0.60 & 11.6 & 0.48 & 4.0 & 180 \\
\hline 26 & 10 & 29 & 61 & 8.5 & 0.35 & 7.5 & 0.63 & 6.5 & 180 \\
\hline 27 & 27 & 43 & 30 & 8.2 & 0.50 & 7.3 & 0.34 & 6.5 & 275 \\
\hline 28 & 30 & 30 & 40 & 8.3 & 0.38 & 14.8 & 0.40 & 3.3 & 140 \\
\hline 29 & 28 & 45. & 27 & 8.2 & 0.35 & 6.8 & 0.43 & 6.5 & 155 \\
\hline 30 & 28 & $45^{\circ}$ & 27 & 8.3 & 0.80 & 5.7 & 0.50 & 3.1 & 360 \\
\hline 31 & - & - & - & - & - & - & - & - & - \\
\hline 32 & 24 & 41 & 35 & 8.5 & 0.62 & 6.2 & 0.49 & 4.0 & 330 \\
\hline Nangarhan & & & & & & & & & \\
\hline 1 & 10 & 10 & 80 & 8.6 & 0.24 & 9.0 & 0.37 & 6.76 & 130 \\
\hline 2 & 17 & 23 & 60 & 8.5 & 0.29 & 13.9 & 0.25 & 6.74 & 125 \\
\hline 3 & 20 & 29 & 51 & 8.5 & 0.80 & 18.0 & 0.08 & 5.7 & 200 \\
\hline 4 & 25 & 19 & 56 & 8.6 & 0.30 & 15.0 & 0.06 & 2.1 & 185 \\
\hline 5 & 25 & 27 & 48 & 8.6 & 0.30 & 4.5 & 0.28 & 9.3 & 200 \\
\hline 6 & 25 & 26 & 49 & 8.2 & 0.10 & 14.3 & 0.40 & 1.8 & 105 \\
\hline 7 & 22 & 38 & 40 & 8.5 & 1.10 & 17.5 & 0.30 & 2.2 & 235 \\
\hline 8 & 30 & 30 & 40 & 8.4 & 0.92 & 10.6 & 0.56 & 2.0 & 165 \\
\hline 9 & 27 & 38 & 35 & 8.5 & 0.82 & 5.2 & 0.58 & 2.2 & 105 \\
\hline 10 & 16 & 10 & 74 & 7.8 & 0.50 & 9.5 & 0.28 & 2.9 & 220 \\
\hline
\end{tabular}




\begin{tabular}{|c|c|c|c|c|c|c|c|c|c|}
\hline \multirow{2}{*}{$\begin{array}{l}\text { Province } \\
\text { s.no. }\end{array}$} & \multicolumn{3}{|c|}{ Texture } & \multirow{2}{*}{$\begin{array}{l}\mathrm{pH} \\
1: 5\end{array}$} & \multirow{2}{*}{ Cond. } & \multirow{2}{*}{$\begin{array}{r}\mathrm{C}_{2} \mathrm{CO}_{3} \\
8 \\
\end{array}$} & \multirow{2}{*}{$\begin{array}{c}0 . \text { Carbon } \\
\%\end{array}$} & \multicolumn{2}{|c|}{ Available } \\
\hline & $\begin{array}{l}\text { Clay } \\
\text { \% }\end{array}$ & $\begin{array}{c}\text { Silt } \\
0 \\
0\end{array}$ & $\begin{array}{l}\text { Sand } \\
\%\end{array}$ & & & & & $\mathrm{P} 1 \mathrm{~b} / \mathrm{a}$ & $\mathrm{K}_{2} \mathrm{O} \quad \mathrm{lb} / \mathrm{a}$ \\
\hline \multicolumn{2}{|c|}{ Nangarhar } & & & & & & & & $\therefore \therefore$ \\
\hline 11 & 25 & 30 & 45 & 7.8 & 0.48 & 7.9 & 0.20 & 2.8 & 105 \\
\hline 12 & 18 & 15 & 67 & 8.4 & 0.30 & 14.3 & 0.33 & 2.8 & 105 \\
\hline 13 & 22 & 36 & 42 & 8.1 & 0.45 & 15.0 & 0.45 & 2.8 & 165 \\
\hline 14 & 19 & 20 & 61 & 8.3 & 0.30 & 24.4 & 0.50 & 2.8 & 105 \\
\hline 15 & 10 & 15 & 75 & 8.7 & 0.20 & 8.2 & 0.12 & 2.0 & 210 \\
\hline 16 & 13 & 23 & 64 & 8.2 & 0.40 & 8.4 & 0.18 & 2.4 & 230 \\
\hline 17 & 10 & 10 & 80 & 8.1 & 0.60 & 6.4 & 0.28 & 2.5 & 235 \\
\hline 18 & 20 & 20 & 60 & 8.0 & 0.55 & 11.4 & 0.30 & 2.5 & 200 \\
\hline 19 & 11 & 15 & 74 & 8.4 & 0.43 & 8.9 & 0.65 & 2.8 & 100 \\
\hline 20 & 21 & 24 & 55 & 8.3 & 0.40 & 6.3 & 0.44 & 4.7 & 270 \\
\hline 21 & 26 & 30 & 44 & 8.3 & 0.60 & 6.2 & 0.49 & 7.8 & 165 \\
\hline 22 & 22 & 29 & 49 & 8.3 & 0.50 & 12.2 & 0.46 & 8.25 & 290 \\
\hline 23 & 22 & 30 & 48 & 8.4 & 0.30 & 16.8 & 0.45 & 6.74 & 180 \\
\hline 24 & 22 & 35 & 43 & 8.6 & 0.50 & 8.5 & 0.37 & 3.35 & 130 \\
\hline 25 & - & - & - & - & - & - & - & - & - \\
\hline 26 & 20 & 23 & 57 & 8.0 & 0.40 & 7.2 & 0.50 & 1.8 & 130 \\
\hline 27 & 22 & 28 & 50 & 8.1 & 0.35 & 5.1 & 0.39 & 2.8 & 275 \\
\hline 28 & 22 & 28 & 50 & 8.4 & 0.25 & 8.4 & 0.59 & 1.8 & $270^{\circ}$ \\
\hline 29 & 24 & 30 & 46 & 8.3 & 0.70 & 8.8 & 0.16 & 4.7 & 190 \\
\hline 30 & 27 & 32 & 41 & 8.6 & 1.00 & 6.8 & 0.37 & 7.8 & 125 \\
\hline 31 & 20 & 22 & 58 & 8.2 & 1.10 & 12.0 & 0.16 & 10.0 & 325 \\
\hline 32 & 26 & 32 & 42 & 8.6 & 1.1 & 10.6 & 0.19 & 2.3 & 260 \\
\hline 33 & 12 & 22 & 76 & 8.6 & 0.2 & 9.0 & 0.23 & 2.8 & 90 \\
\hline 34 & 22 & 30 & 48 & 8.7 & 0.3 & 9.1 & 0.32 & 2.4 & 175 \\
\hline 35 & 18 & 22 & 60 & 8.3 & 0.5 & 10.5 & 0.20 & 2.8 & 125 \\
\hline 36 & 14 & 25 & 61 & 8.4 & 0.5 & 9.9 & 0.44 & 2.6 & 110 \\
\hline 37 & 20 & 22 & 58 & 8.7 & 0.3 & 9.0 & 0.35 & 1.8 & 185 \\
\hline 38 & 11 & 19 & 70 & 8.6 & 0.8 & 10.4 & 0.37 & 7.5 & 100 \\
\hline 39 & 12 & 20 & 68 & 8.5 & 0.9 & 11.9 & 0.28 & 1.7 & 100 \\
\hline 40 & 14 & 22 & 64 & 7.8 & 0.6 & 8.1 & 0.33 & 3.8 & 125 \\
\hline 41 & - & - & - & - & - & - & - & - & - \\
\hline 42 & 11 & 16 & 73 & 8.3 & 0.4 & 9.5 & 0.37 & 8.8 & 150 \\
\hline 43 & 18 & 25 & 57 & 8.5 & 0.2 & 11.4 & 0.11 & 8.9 & 100 \\
\hline 44 & 17 & 20 & 63 & 8.4 & 0.7 & 13.0 & 0.23 & 10.5 & 140 \\
\hline 45 & 20 & 25 & 55 & 8.0 & 0.80 & 9.8 & 0.21 & 8.4 & 150 \\
\hline 46 & 12 & 38 & 50 & 8.6 & 0.90 & 9.9 & 0.12 & 8.3 & 145 \\
\hline
\end{tabular}




\begin{tabular}{|c|c|c|c|c|c|c|c|c|c|}
\hline \multirow{2}{*}{$\begin{array}{l}\text { Province } \\
\text { o.no. }\end{array}$} & \multicolumn{3}{|c|}{ ‥ Toxture } & \multirow{2}{*}{$\begin{array}{l}2 E^{2} \\
1: 5\end{array}$} & \multirow{2}{*}{$\begin{array}{l}\text { Cond. } \\
\mathrm{man} / 25^{\circ} \circ\end{array}$} & \multirow{2}{*}{$\begin{array}{c}\mathrm{CaCO}_{3} \\
\not \\
\end{array}$} & \multirow{2}{*}{$\begin{array}{c}0 . \text { Cerrbon } \\
8 \\
\end{array}$} & \multicolumn{2}{|c|}{ Available } \\
\hline & $\begin{array}{c}\text { Clay } \\
8 \\
0\end{array}$ & $\begin{array}{c}\text { Silt } \\
\%\end{array}$ & $\begin{array}{l}\text { Sand } \\
0 \\
0\end{array}$ & & & & & $\mathrm{PIb} / \mathrm{a}$ & $\mathrm{K}_{2} \mathrm{O} \mathrm{lb} / \mathrm{a}$ \\
\hline Wangarhat & & & & & & & & & \\
\hline$\Delta T$ & 18 & 26 & 56 & 8.5 & 1.20 & 10.5 & 0.44 & 6.9 & 185 \\
\hline 48 & 16 & 38 & 46 & 8.2 & 0.25 & 106 & 0.30 & 7.8 & 135 \\
\hline 49 & 18 & 22 & 60 & 8.4 & 0.50 & 8.4 & 0.46 & 8.0 & 130 \\
\hline 50 & 22 & 23 & 55 & 8.5 & 0.50 & 20.3 & 0.38 & 9.0 & 200 \\
\hline $\begin{array}{r}\text { Lagman } \\
1\end{array}$ & 10.8 & 38.0 & 51.2 & $7 \cdot 3$ & 0.5 & 2.58 & 0.12 & 4.75 & 260 \\
\hline 2 & 9 & 26 & 75 & 8.3 & 1.4 & 2.4 & 0.54 & 1.75 & 130 \\
\hline 3 & 12 & 40 & 48 & 7.8 & 2.1 & 1.9 & 0.27 & $3 . \infty 0$ & 155 \\
\hline 4 & 35 & 46 & 40 & 7.8 & 0.8 & 2.6 & 0.50 & 2.50 & 130 \\
\hline 5 & 13 & 37 & 50 & $7.7^{\circ}$ & 2.1 & 2.8 & 0.17 & 2.75 & 165 \\
\hline 5 & 2.4 & 36 & 50 & $\because .0$ & 2.2 & 0.4 & 0.12 & 1.75 & 245 \\
\hline 7 & 14 & 46 & 43 & 7.8 & $\therefore .7$ & 2.3 & 0.36 & 4.0 & 250 \\
\hline 8 & 15 & 37 & 18 & 7.6 & $\therefore .3$ & 0.9 & 0.96 & 4.8 & 285 \\
\hline 3 & 9 & 24 & 67 & 7.6 & 3.7 & 2.3 & 0.36 & 6.5 & 195 \\
\hline 10 & 13 & 40 & 47 & 7.0 & 1.2 & 3.5 & 0.46 & 2.75 & 230 \\
\hline 11 & 19 & 36 & 45 & 8.1 & 0.9 & 0.7 & 0.36 & 4.75 & 255 \\
\hline 12 & 13 & 44 & 43 & 8.5 & 1.0 & 2.5 & 0.22 & 2.75 & 230 \\
\hline 13 & 10 & 38 & 52 & 7.9 & 0.9 & 0.7 & 0.40 & 6.50 & 220 \\
\hline 14 & 13 & 40 & 47 & 8.5 & 0.8 & 0.6 & 0.47 & 1.00 & 125 \\
\hline 15 & 14 & 26 & 60 & 7.5 & 0.9 & 0.7 & 0.32 & 1.50 & 135 \\
\hline 16 & 11 & 36. & 53 & 6.8 & 1.0 & 0.7 & 0.37 & 2.00 & 165 \\
\hline 17 & 15 & $42^{\circ}$ & 43 & 7.3 & 0.6 & 0.9 & 0.50 & 3.00 & 157 \\
\hline 18 & 13 & 34 & 53 & 7.6 & 1.5 & 2.3 & 0.64 & 8.50 & 130 \\
\hline 19 & 9 & 38 & 53 & 7.8 & 0.4 & 3.7 & 0.48 & 1.00 & 100 \\
\hline 20 & 12 & 34 & 54 & 7.0 & 0.8 & 1.1 & 0.49 & 1.75 & 175 \\
\hline 21 & 15 & 32 & 53 & 7.0 & 1.9 & 0.9 & 0.11 & $8 . \infty 0$ & 137 \\
\hline 22 & 11 & 43 & 46 & 7.2 & 1.4 & 0.7 & 0.45 & 4.00 & 160 \\
\hline 23 & 15 & 36 & 49 & 7.5 & 1.0 & 0.4 & 0.11 & 2.0 & 170 \\
\hline 24 & 16 & 24 & 60 & 7.5 & 1.1 & 0.5 & 0.14 & 4.0 & 190 \\
\hline 25 & 10 & 18 & 72 & 7.6 & 0.8 & 3.5 & 0.18 & 1.4 & 120 \\
\hline 26 & 10 & 34 & 48 & 7.0 & 1.3 & 1.1 & 0.32 & 1.4 & 190 \\
\hline 27 & 13 & 40 & 47 & 8.0 & 1.4 & 1.7 & 0.49 & 2.5 & 155 \\
\hline 28 & 18 & 38 & 44 & 7.5 & 1.1 & 3.9 & 0.30 & 1.5 & 195 \\
\hline
\end{tabular}




\begin{tabular}{|c|c|c|c|c|c|c|c|c|c|}
\hline \multirow{2}{*}{$\begin{array}{l}\text { Province } \\
\text { s.no. }\end{array}$} & \multicolumn{3}{|c|}{ Texture } & \multirow{2}{*}{$\begin{array}{l}\mathrm{pH} \\
\mathrm{l}: 5\end{array}$} & \multirow{2}{*}{ Cond. } & \multirow{2}{*}{$\begin{array}{c}\mathrm{CaCO}_{3} \\
\stackrel{8}{8} \\
\end{array}$} & \multirow{2}{*}{$\begin{array}{c}\text { O. Carbon } \\
\%\end{array}$} & \multicolumn{2}{|c|}{ Available } \\
\hline & Clay & $\underset{\%}{\operatorname{silt}}$ & $\begin{array}{c}\text { Sand } \\
o\end{array}$ & & & & & PIb/a & $\mathrm{K}_{2} \mathrm{O} \quad \mathrm{lb} / \mathrm{a}$ \\
\hline Lagman 29 & 10 & 37 & 53 & 7.6 & 1.06 & 1.2 & 0.15 & 2.7 & 175 \\
\hline 30 & 10 & 36 & 54 & 8.0 & 1.4 & 1.1 & 0.18 & 2.0 & \\
\hline 31 & 10 & 32 & 58 & $7 \cdot 5$ & 0.8 & 2.5 & 0.22 & 2.75 & 155 \\
\hline 32 & 10 & 32 & 58 & 8.1 & 1.2 & 1.2 & 0.54 & 2.00 & 180 \\
\hline 33 & 12 & 36 & 52 & 8.4 & 1.0 & 1.4 & 0.47 & 1.50 & 180 \\
\hline 34 & 13 & 37 & 50 & 7.8 & 1.5 & 0.8 & 0.48 & 2.75 & 105 \\
\hline 35 & 13 & 30 & 57 & 7.0 & 0.8 & 0.9 & 0.59 & 5.5 & 120 \\
\hline 36 & 13 & 36 & 51 & 8.0 & 0.8 & 1.0 & 0.14 & 1.5 & 150 \\
\hline 37 & 20 & 37 & 43 & 8.0 & 0.7 & 0.7 & 0.48 & 11.5 & 165 \\
\hline 38 & 10 & 20 & 70 & 7.2 & 0.9 & 0.8 & 0.49 & 8.0 & 145 \\
\hline 39 & 15 & 30 & 55 & 8.0 & 1.5 & 2.4 & 0.18 & 7.8 & 150 \\
\hline 40 & 15 & 32 & 53 & 6.9 & 0.8 & 3.7 & 0.10 & 4.0 & 170 \\
\hline 41 & 15 & 41 & 44 & 7.5 & 0.9 & 6.6 & 0.14 & 6.5 & 155 \\
\hline 42 & 15 & 32 & 53 & 8.2 & 1.1 & 1.8 & 0.26 & 1.5 & 165 \\
\hline 43 & 16 & 34 & 50 & 7.0 & 0.7 & 3.02 & 0.60 & 2.8 & 155 \\
\hline 44 & 15 & 40 & 45 & 8.0 & 0.8 & 0.9 & 0.85 & 4.0 & 175 \\
\hline 45 & 12 & 36 & 52 & 7.0 & 0.7 & 0.4 & 0.42 & 2.75 & 195 \\
\hline 46 & 10 & 38 & 52 & 8.0 & 0.9 & 0.2 & 0.50 & 5.5 & 300 \\
\hline 47 & 17 & 38 & 45 & 8.0 & 0.8 & 0.4 & 0.30 & 11.5 & 210 \\
\hline 48 & 16 & 38 & 46 & 8.0 & 0.8 & 1.0 & 0.11 & 3.5 & 170 \\
\hline 49 & 16 & 38 & 46 & 7.5 & 0.9 & 4.1 & 0.50 & 4.0 & 180 \\
\hline 50 & 18 & 37 & 55 & 7.3 & 0.9 & 2.7 & 0.42 & 10.5 & 190 \\
\hline
\end{tabular}


APPANDIY III

SOIL ANALYSIS DATA

(d) Corm $-1967-1968$

\begin{tabular}{|c|c|c|c|c|c|c|c|c|}
\hline \multirow[b]{2}{*}{ Soil Prop. } & \multicolumn{2}{|c|}{ Charak } & \multicolumn{2}{|c|}{$\begin{array}{l}\text { Qualei Buba } \\
\text { Pathkewa }\end{array}$} & \multicolumn{2}{|c|}{ Qualai Baw } & \multirow{2}{*}{\multicolumn{2}{|c|}{$\frac{\text { Qualai Mojahale }}{\text { L. }} \frac{\text { M-13 }}{M-1}$}} \\
\hline & L. & $X_{-13}$ & I... & $M-13$ & L. & $M-13$ & & \\
\hline \multicolumn{9}{|l|}{ Texture } \\
\hline $1 \% \mathrm{Cl}$ ay & 26.8 & 26.8 & 30.8 & 30.8 & 27.2 & 30.0 & 30.0 & 32.8 \\
\hline Stint & 32.0 & 34.0 & $\begin{array}{l}34.0 \\
30.0\end{array}$ & 26.0 & 22.0 & 30.0 & 30.0 & 38.0 \\
\hline$\% \operatorname{San} \alpha$ & 44.2 & 44.2 & $\begin{array}{l}45.2 \\
43.2\end{array}$ & 43.2 & 50.8 & 40.0 & 40.0 & 39.2 \\
\hline pin i: 1 & 6.5 & 8.2 & 8.1 & 8.2 & 8.2 & 8.4 & 8.4 & 8.9 \\
\hline$p h 4: 5$ & $0 . \%$ & 8.5 & 8.6 & 3.5 & 8.5 & 8.7 & $8 . \pi$ & 3.4 \\
\hline $\begin{array}{l}\text { ic. cona. } \\
\text { inimings/cm } \\
\text { et } 25^{\circ} \mathrm{c}\end{array}$ & $0 . \hat{i}$ & 0.1 & $\begin{array}{l}0.02 \\
0.02\end{array}$ & 0.02 & 0.03 & 0.04 & 0.04 & 0.01 \\
\hline $\mathrm{CaCO}_{3} \%$ & 15.1 & 15.1 & $\begin{array}{l}14.1 \\
16.4\end{array}$ & 17.5 & 19.1 & 19.0 & 19.0 & $17 \cdot 9$ \\
\hline Org. c & 0.67 & 0.67 & $\begin{array}{l}0.51 \\
0.78\end{array}$ & 0.26 & 0.29 & 0.42 & 0.42 & 0.49 \\
\hline Avl. N & 392 & 392 & $\begin{array}{l}336 \\
350\end{array}$ & 218 & 240 & 182 & 182 & 212 \\
\hline Avl. P & 0.0 & 0.0 & $\begin{array}{l}16.1 \\
18.8\end{array}$ & 0.0 & 0.4 & 18.4 & 18.4 & 18.4 \\
\hline Avl. $\mathrm{K}_{2} \mathrm{O}$ & 440 & 440 & $\begin{array}{l}350 \\
370\end{array}$ & 400 & 450 & 450 & 450 & 480 \\
\hline
\end{tabular}


APPEINDIX III

SOIL ANAIYSIS DATA

(e) Corn - $1968-1969$

Paktya Province

\begin{tabular}{|c|c|c|c|c|c|c|}
\hline $\begin{array}{l}\text { S.No } \\
\text { Village }\end{array}$ & $\begin{array}{l}\mathrm{pH} \\
1: 5\end{array}$ & $\begin{array}{l}\text { Elc. Cond } \\
\text { manhos/on }\end{array}$ & $\mathrm{CaCO}_{3}^{-} \%$ & $\underset{\%}{\text { Org. Carbon }}$ & $\begin{array}{l}\text { Available } \\
\text { P 1b/a }\end{array}$ & $\begin{array}{l}\text { Available } \\
\mathrm{K}_{2} \mathrm{O} 1 \mathrm{~b} / \mathrm{a}\end{array}$ \\
\hline 1. Kalago & 8.5 & 0.70 & 15.5 & 0.20 & 5.5 & 188 \\
\hline 2. Kalago & 8.5 & 0.90 & 16.5 & 0.50 & 4.8 & 124 \\
\hline 3. Margane & 8.5 & 1.2 & 15.0 & 0.30 & 4.5 & 200 \\
\hline 4. Kalage & 8.3 & 1.0 & 16.0 & 0.40 & 4.5 & 280 \\
\hline 5. Kalago & 8.6 & 0.7 . & 17.5 & 0.44 & 2.1 & 100 \\
\hline 6. Kalago & 8.4 & 1.8 & 15.0 & 0.33 & 4.6 & 100 \\
\hline 7. Kaghatia & 8.0 & 1.5 & 15.7 & 0.50 & 4.8 & 156 \\
\hline 8. Kal ago & 8.7 & 1.5 & 11.2 & 0.75 & 2.5 & 252 \\
\hline 9. Kalago & 8.4 & 1.0 & 14.2 & 0.30 & 16.0 & 100 \\
\hline 10.Kalago & 8.5 & 1.0 & 12.5 & 0.50 & - & 160 \\
\hline 11. Razan khial & 8.4 & 0.8 & 15.0 & 0.62 & 5.4 & 148 \\
\hline 12.Much & 8.5 & 0.9 & 16.0 & 0.20 & 5.2 & 156 \\
\hline 13.Much & 8.5 & 0.6 & 17.7 & 0.50 & 4.7 & 108 \\
\hline 14.Salem khial & 8.2 & 0.8 & 20.0 & 0.25 & 5.6 & 80 \\
\hline 15.Peroz khial & 8.3 & 0.25 & - & 0.35 & 5.6 & 85 \\
\hline 16. Chenow & 8.1 & 0.7 & 15.0 & 0.75 & 4.2 & 46 \\
\hline 17. Husan khial & 8.3 & 0.7 & - & 0.17 & 4.8 & 46 \\
\hline 18. Khaud khial & 8.2 & 0.9 & 19.7 & 0.48 & 4.8 & 132 \\
\hline 19. Chenow & 8.1 & 0.25 & 8.6 & 0.72 & 6.0 & 56 \\
\hline $\begin{array}{c}\text { 20.Saidi Khoja } \\
\text { Gardez }\end{array}$ & 8.2 & 1.0 & 19.0 & 0.7 & 5.4 & 176 \\
\hline 21. Khoja Husen & 8.4 & 0.8 & 9.75 & 0.49 & 1.2 & 110 \\
\hline 22. Quali Saida & 8.1 & 0.9 & 20.7 & 0.27 & 4.9 & - \\
\hline 23.Gardez & 8.4 & 1.8 & 20.1 & 0.36 & 6.0 & 96 \\
\hline Logar Province & & & & & & \\
\hline 1. Puljigi & 8.4 & 0.8 & $24 \cdot 7$ & 0.49 & 4.0 & 180 \\
\hline 2. Puljigi & 8.3 & 0.7 & 20.5 & 0.68 & 8.2 & 120 \\
\hline 3. Q. Bawa & 8.2 & 2.0 & 20.5 & 0.39 & 5.8 & 156 \\
\hline 4. Q. Bawa & 8.0 & 1.2 & 20.5 & 0.15 & 4.8 & 118 \\
\hline 5. Jauli Shakh & 8.4 & 1.2 & 14.7 & 0.70 & 5.8 & 84 \\
\hline 6. Jauli Shakh & 8.4 & 0.9 & 19.2 & 0.42 & 5.6 & 152 \\
\hline
\end{tabular}


Logar Province (continued)

\begin{tabular}{|c|c|c|c|c|c|c|}
\hline $\begin{array}{l}\text { S. No. } \\
\text { Village }\end{array}$ & $\begin{array}{l}\mathrm{pH} \\
1: 5\end{array}$ & $\begin{array}{l}\text { ilc. Cond } \\
\mathrm{mmhos} / \mathrm{cm}\end{array}$ & $\mathrm{CaCO}_{3} \%$ & Org. Carbon & $\begin{array}{l}\text { Available } \\
\text { P lb/a }\end{array}$ & $\begin{array}{l}\text { hvailable } \\
\mathrm{K}_{2} \mathrm{O} \mathrm{lb} / \mathrm{a}\end{array}$ \\
\hline 7. Deh Shaikh & 8.5 & 1.0 & 23.0 & 0.29 & 5.2 & 112 \\
\hline 8. Deh Shaikh & 8.5 & 1.0 & 22 & 0.64 & $4 \cdot 9$ & 11.4 \\
\hline 9. Baraki Barak & 8.3 & 0.85 & 19.2 & 0.44 & 5.2 & 144 \\
\hline 10. Baraki Barak & 8.4 & 0.95 & 12.7 & 0.37 & 6.0 & 88 \\
\hline 11. Patkhabi & 8.5 & 1.0 & 14.5 & 0.50 & 4.8 & 176 \\
\hline 12. Deh shaikh & 8.5 & 0.7 & - & 0.48 & 5.4 & 160 \\
\hline 13. Omi Sarda & 8.3 & $1 \cdot 1$ & 19.75 & 0.27 & 5.6 & 160 \\
\hline 14. Reti-hab & 8.4 & 0.75 & 20.0 & 0.72 & 5.8 & 92 \\
\hline 15. Dosharibi & 8.4 & 0.70 & 24.0 & 0.42 & 11.6 & 92 \\
\hline 16. 2. Patheb & 3.4 & 0.70 & 10.2 & 0.47 & 4.8 & 76 \\
\hline 17. Berexi Eerek & $8 .:$ & 2.0 & $\because \because$ & 0.0 & 4.2 & 7 \\
\hline 18, Werüatcolale & 8.6 & 0.62 & 10.2 & 0,30 & 3.72 & $10:$ \\
\hline 1: B. Barar & 2.5 & 0.70 & $\therefore 5$ & 0.70 & $\therefore 3$ & 8 \\
\hline 20. E. Berag & 8.3 & 1.2 & $20 \cdot 5$ & 0.33 & 3.0 & 130 \\
\hline 21. Zaqankhiai & 8.4 & 2.2 & 19.0 & 0.43 & 4.6 & 216 \\
\hline 22. Zaugmkhial & 8.2 & 1.5 & 22.0 & 0.68 & 4.88 & 244 \\
\hline 23. Shikhan & 8.4 & 1.0 & - & 0.28 & 5.4 & 160 \\
\hline 24. Nazarqualai & 8.3 & 1.0 & $24 \cdot 0$ & 0.20 & $4 \cdot 3$ & 96 \\
\hline 25. Abrahamkhial & 8.3 & 0.8 & 17.0 & 0.75 & 12.8 & 124 \\
\hline 26. Qalai Baba & $8: 5$ & 0.7 & 22.25 & 0.37 & 6.36 & 188 \\
\hline 27. Qalai Baba & 8,4 & 0.7 & 24.7 & 0.49 & 6.8 & 176 \\
\hline 28. Patkhabi & 8.3 & 0.85 & 13.0 & 0.64 & 5.28 & 148 \\
\hline 29. Balkhshabad & 8.3 & 0.80 & 22.0 & 0.67 & 6.08 & 96 \\
\hline 30. Patkhabi & 8.5 & 0.80 & 20.0 & 0.48 & 5.6 & 112 \\
\hline 31. Barakirajan & 8.4 & 1.6 & & 0.35 & 5.2 & 256 \\
\hline 32. Q. Zarin & 8.4 & 0.9 & 17.5 & 0.32 & 4.4 & 72 \\
\hline 33. Shamzar & 7.9 & 0.9 & 24.0 & 0.70 & $4 \cdot 5$ & 88 \\
\hline 34. Pandch & 8.2 & 0.8 & 20.50 & 0.24 & 1.0 & 92 \\
\hline 35. Dabar & 8.5 & 1.1 & 15.25 & 0.13 & 6.2 & 152 \\
\hline 36. Charak & 8.4 & 2.1 & 18.0 & 0.70 & 7.6 & 192 \\
\hline 37. Bagchimalik & 8.4 & 1.0 & 18.0 & 0.18 & 5.0 & 120 \\
\hline 38. Shaha Qula & 8.4 & 0.75 & 16.0 & 0.15 & 3.68 & 116 \\
\hline 39. Bagchimalik & 8.4 & 1.0 & 23.0 & 0.20 & 4.8 & 96 \\
\hline 40. Charak & 8.5 & 1.0 & 20.0 & 0.48 & 5.0 & 150 \\
\hline
\end{tabular}


Logar Province (continued)

\begin{tabular}{|c|c|c|c|c|c|c|}
\hline $\begin{array}{c}\text { S No. } \\
\text { Village }\end{array}$ & $\begin{array}{c}\mathrm{pH} \\
1: 5\end{array}$ & $\begin{array}{c}\text { Elc.Cond } \\
\text { mnhos/cm }\end{array}$ & $\mathrm{CaCO}_{3} . \%$ & $\begin{array}{c}\text { Org. Carbon } \\
\%\end{array}$ & $\begin{array}{c}\text { Available } \\
\mathrm{P} \mathrm{lb/a}\end{array}$ & $\begin{array}{c}\text { Available } \\
\mathrm{K}_{2} \mathrm{Ob} / \mathrm{a}\end{array}$ \\
\hline 41. Shanganak & 8.5 & 0.7 & 22.5 & 0.39 & 5.2 & 104 \\
42. Kolangar & 8.4 & 0.8 & 18.7 & 0.49 & 5.68 & 100 \\
43. Kolangar & 8.4 & 0.5 & 18.0 & 0.46 & 6.08 & 124 \\
44. Kolangar & 8.3 & 0.2 & 19.0 & 0.39 & 5.0 & 120 \\
45. Khojahasan & 8.3 & 0.5 & 16.0 & 0.24 & 6.3 & 76 \\
46. Khojahasan & 8.3 & 0.7 & 11.0 & 0.50 & 5.2 & 120 \\
& & & & & & \\
\hline
\end{tabular}


APFENDIX III

SOIL ARALYSIS DATA

(f) Rice- $1967-1968$

\begin{tabular}{|c|c|c|c|c|c|c|c|c|}
\hline \multirow{2}{*}{ S. No. Soil Prop. } & \multicolumn{4}{|c|}{ LACORAN } & \multicolumn{4}{|c|}{ NBNGGARHAR } \\
\hline & Shmati & Alishung & Mireari & Chardy & Bensood & Nawabad & Derinoor & $\begin{array}{l}\text { Dorral } \\
\text { Mhake }\end{array}$ \\
\hline 1. Clay $\%$ & 13.4 & 22.4 & 26.4 & 20.0 & 26.4 & 26.4 & 26.4 & 24.0 \\
\hline 2. Silt $\%$ & 30.0 & 30.0 & 28.0 & 30.0 & 26.0 & 26.0 & 26.0 & 22.4 \\
\hline 3. Sand \% & 51.6 & 47.6 & 45.6 & 50.0 & 47.6 & 47.6 & 47.6 & 53.6 \\
\hline 4. $\mathrm{C}_{\mathrm{a}} \mathrm{C}_{0} 3 \%$ & 0.75 & 0.75 & 1.0 & 1.25 & 0.75 & 0.50 & 0.50 & $0.7=$ \\
\hline 5. $\mathrm{pH} 1: 1$ & 7.5 & 7.6 & $7 \cdot 3$ & 8.3 & 7.6 & 7.6 & 7.5 & 7.4 \\
\hline 6. $\quad \mathrm{pH} \quad 1: 5$ & 8.4 & 7.9 & 8.2 & 8.6 & 8.1 & 8.0 & 7.8 & 5.0 \\
\hline $\begin{array}{l}\text { 7. Elec, cond. } \\
\text { mmhos } / \mathrm{cm}, 25 \mathrm{c} \text {. }\end{array}$ & 0.02 & 0.03 & 6.02 & 0.01 & 0.03 & 0.03 & 0.01 & 0.02 \\
\hline 8. Orenic C. $\%$ & 0.39 & 0.51 & 2.80 & 0.16 & 0.69 & 0.86 & 0.20 & 0.28 \\
\hline 9. Avil P. Ib/a & 4.4 & 2.4 & 6.4 & 5.6 & 26.6 & 20.4 & 4.0 & 28.4 \\
\hline 10. Aril. $\mathrm{K}_{2} \mathrm{O} \mathrm{IO} / \mathrm{a}$ & 380 & 180 & 392 & 98 & 192 & 284 & 95 & 80 \\
\hline 11. Avil. $N$ Ib/a & 180 & 280 & 255 & 180 & 364 & 320 & 194 & 220 \\
\hline
\end{tabular}


APPENDIX III

SOIL ANALYSIS DATA

(g) Sugarbeet - 1967-1968

\begin{tabular}{|c|c|c|c|c|c|c|c|}
\hline \multirow[b]{2}{*}{ S.No. } & \multirow[b]{2}{*}{ Soil Prop. } & \multicolumn{6}{|c|}{$\mathrm{L} O \mathrm{CA} \mathrm{AT} I O \mathrm{~N}$} \\
\hline & & Baladari & Tarakhia! & Sharabi tapa & Kohna & $\begin{array}{c}\text { Near } \\
\text { Nangahar }\end{array}$ & $\begin{array}{l}\text { Baladari } \\
\text { III }\end{array}$ \\
\hline 1 & Texture & & & & & & \\
\hline & Clay $\%$ & 31 & 27 & 31 & 19 & 23 & 23 \\
\hline & Silt $\%$ & 33 & 31 & 29 & 30 & 24 & 22 \\
\hline & Sand of & 36 & 42 & 40 & 51 & 53 & 55 \\
\hline 2 & Cal.carb. $\%$ & 17.9 & 17.9 & 17.8 & 13.6 & 17.6 & 18.6 \\
\hline 3 & $\mathrm{pH} \quad 1: 1$ & 8.2 & $8.1^{\circ}$ & 8.2 & 8.1 & 8.1 & 8.1 \\
\hline & $1: 5$ & 8.2 & 8.3 & 8.3 & 8.3 & 8.4 & 8.4 \\
\hline 4 & $\begin{array}{l}\text { Elec. Cond. mmhos/ } \\
\mathrm{cm} \text {. at } 25 \mathrm{c} \text {. }\end{array}$ & 0.15 & 0.16 & 0.14 & 0.17 & 0.18 & 0.15 \\
\hline 5 & Org. Carbon $\%$ & 0.38 & 0.22 & 0.19 & 0.31 & 0.40 & 0.27 \\
\hline 6 & Avil. N. Ib/a & 136 & 192 & 250 & 175 & 200 & 225 \\
\hline 7 & Avil. P. Ib/a & 20 & 12 & 20 & 16 & 11 & 14 \\
\hline 8 & Avil. $\mathrm{K}_{2} \mathrm{O} \mathrm{lb} / \mathrm{a}$ & 290 & 250 & 260 & 130 & 275 & 280 \\
\hline
\end{tabular}




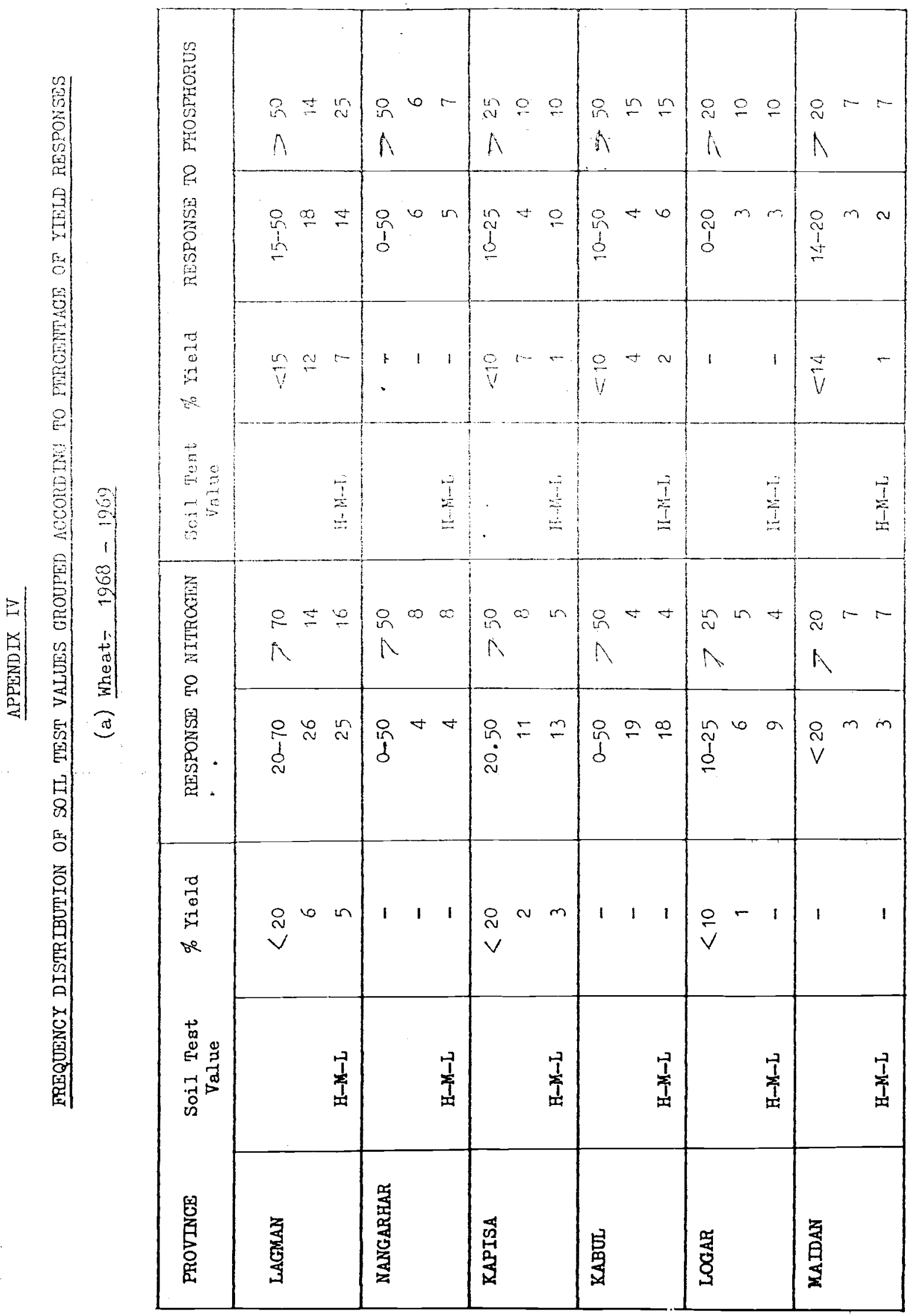


APPENDIX IV

FREQUENCY DISTRIBUTION OF SOTL TEST VALUES GROUPED ACCORD ING TO PERCENTAGE OF YIELD RESPONSES

(b) Corn $-1969-.1970$

\begin{tabular}{|c|c|c|c|c|c|}
\hline Province & $\begin{array}{l}\text { Total No. } \\
\text { of samples }\end{array}$ & $\begin{array}{l}\text { Number of } \\
\text { soil samples } \\
\text { falling in } 3 \\
\text { Eroups for } \mathrm{N} . \\
1 /\end{array}$ & $\begin{array}{l}\text { Number of crop } \\
\text { samples falling } \\
\text { in } 3 \text { classes of } \\
\% \text { increases } \\
\text { groups }\end{array}$ & $\begin{array}{l}\text { Number of } \\
\text { soil samples } \\
\text { falling in } 3 \\
\text { groups for } P .\end{array}$ & $\begin{array}{l}\text { Number of } \\
\text { crop samples } \\
\text { falling in } 3 \\
\text { classes of } \% \\
\text { increases } \\
\text { groups }\end{array}$ \\
\hline Paktya & 15 & $\begin{array}{l}\text { Low Med High } \\
123-\end{array}$ & $\begin{array}{l}-0-70,70-135 \\
-\quad 3,12\end{array}$ & $\begin{array}{l}\text { Low Med High } \\
\begin{array}{ccc}14 & 1\end{array}\end{array}$ & $\begin{array}{l}-0-18,18-51 \\
-\quad 1,14\end{array}$ \\
\hline Logar & 36 & $\begin{array}{rrr}- & - & - \\
33 & 3 & -\end{array}$ & $\begin{array}{l}-0-80,80-162 \\
-\quad 3,33\end{array}$ & $\begin{array}{ccc}- & - & - \\
34 & 2 & -\end{array}$ & $\begin{array}{l}-0-15,15-75 \\
-\quad 2,34\end{array}$ \\
\hline
\end{tabular}

1/ \% organic carbon has been used as an index of available nitrogen. 